\title{
Equalities and inequalities for ranks of products of generalized inverses of two matrices and their applications
}

Yongge $\operatorname{Tian}^{*}$

\author{
"Correspondence: \\ yongge.tian@gmail.com \\ China Economics and Management \\ Academy, Central University of \\ Finance and Economics, Beijing, \\ 100081, China
}

\begin{abstract}
A complex matrix $X$ is called an $\{i, \ldots, j\}$-inverse of the complex matrix $A$, denoted by $A^{(i, \ldots, j)}$, if it satisfies the ith, ..., jth equations of the four matrix equations (i) $A X A=A$, (ii) $X A X=X$, (iii) $(A X)^{*}=A X$, (iv) $(X A)^{*}=X A$. The eight frequently used generalized inverses of $A$ are $A^{\dagger}, A^{(1,3,4)}, A^{(1,2,4)}, A^{(1,2,3)}, A^{(1,4)}, A^{(1,3)}, A^{(1,2)}$, and $A^{(1)}$. The $\{i, \ldots, j\}$-inverse of a matrix is not necessarily unique and their general expressions can be written as certain linear or quadratic matrix-valued functions that involve one or more variable matrices. Let $A$ and $B$ be two complex matrices such that the product $A B$ is defined, and let $A^{(i, \ldots . j)}$ and $B^{(i, \ldots . j)}$ be the $\{i, \ldots, j\}$-inverses of $A$ and $B$, respectively. A prominent problem in the theory of generalized inverses is concerned with the reverse-order law $(A B)^{(i, \ldots, j)}=B^{(i, \ldots, j)} A^{(i, \ldots, j)}$. Because the reverse-order products $B^{(i, \ldots . j)} A^{(i, \ldots . j)}$ are usually not unique and can be written as linear or nonlinear matrix-valued functions with one or more variable matrices, the reverse-order laws are in fact linear or nonlinear matrix equations with multiple variable matrices. Thus, it is a tremendous and challenging work to establish necessary and sufficient conditions for all these reverse-order laws to hold. In order to make sufficient preparations in characterizing the reverse-order laws, we study in this paper the algebraic performances of the products $B^{(i, \ldots . j)} A^{(i \ldots ., j)}$. We first establish 126 analytical formulas for calculating the global maximum and minimum ranks of $B^{(i, \ldots . j)} A^{(i, \ldots . j)}$ for the eight frequently used $\{i, \ldots, j\}$-inverses of matrices $A^{(i, \ldots . j)}$ and $B^{(i, \ldots . j)}$, and then use the rank formulas to characterize a variety of algebraic properties of these matrix products.
\end{abstract}

MSC: 15A03; 15A09; 15A24

Keywords: matrix product; generalized inverse; reverse-order law; rank; equality; inequality

\section{Introduction}

Let $A \in \mathbb{C}^{m \times n}$ be a general matrix. The Moore-Penrose inverse of $A$, denoted by $A^{\dagger}$, is defined to be a matrix $X \in \mathbb{C}^{n \times m}$ satisfying the following four Penrose equations:
(i) $A X A=A$,
(ii) $X A X=X$,
(iii) $(A X)^{*}=A X$,
(iv) $(X A)^{*}=X A$.

The concept of the Moore-Penrose inverse was independently described by Moore in [1] and Penrose in [2]. It is well known that $A^{\dagger}$ always exists for any $A$ and is unique; see, e.g., [3-5]. Moreover, a matrix $X$ is called an $\{i, \ldots, j\}$-inverse of $A$, denoted by $A^{(i, \ldots, j)}$, if

(c) 2016 Tian. This article is distributed under the terms of the Creative Commons Attribution 4.0 International License (http://creativecommons.org/licenses/by/4.0/), which permits unrestricted use, distribution, and reproduction in any medium, provided you give appropriate credit to the original author(s) and the source, provide a link to the Creative Commons license, and indicate if changes were made. 
it satisfies the $i$ th, $\ldots, j$ th equations in (1.1). The collection of all $\{i, \ldots, j\}$-inverses of $A$ is denoted by $\left\{A^{(i, \ldots, j)}\right\}$. The eight frequently used generalized inverses of $A$ are

$$
A^{\dagger}, \quad A^{(1,3,4)}, \quad A^{(1,2,4)}, \quad A^{(1,2,3)}, \quad A^{(1,4)}, \quad A^{(1,3)}, \quad A^{(1,2)}, \quad A^{(1)} .
$$

Generalized inverses of matrices are common tools to deal with singular matrices, and now become fruitful and core parts of matrix theory and applications.

Before proceeding, we introduce the natation used in this paper. The symbol $\mathbb{C}^{m \times n}$ stands for the collection of all $m \times n$ complex matrices. The symbols $r(A), \mathscr{R}(A)$, and $\mathscr{N}(A)$ stand for the rank, the range (column space, and the kernel (null space) of a matrix $A \in \mathbb{C}^{m \times n}$, respectively. $I_{m}$ denotes the identity matrix of order $m ;[A, B]$ denotes a row block matrix consisting of $A$ and $B$. We use $E_{A}=I_{m}-A A^{\dagger}$ and $F_{A}=I_{n}-A^{\dagger} A$ to stand for the two projectors induced by $A$.

In matrix theory, a fundamental matrix operation is to find the inverse of a square matrix when it is nonsingular, or to find generalized inverses of the matrix when it is singular. It is a common fact that for a pair of nonsingular matrices $A$ and $B$ of the same size, the product $A B$ is nonsingular as well, and the ordinary inverse of $A B$ can be expressed as $(A B)^{-1}=B^{-1} A^{-1}$. This basic algebraic equality is called the reverse-order law of the inverse of the product of two nonsingular matrices in linear algebra. This law shows that if both $A^{-1}$ and $B^{-1}$ are given, we can use their product $B^{-1} A^{-1}$ instead of $(A B)^{-1}$, so that this law can be used to simplify various matrix expressions that involve inverse operations of products of nonsingular matrices. Because generalized inverses of matrices are extensions of ordinary inverses of matrices, it is natural to accordingly construct a reverse-order law

$$
(A B)^{(i, \ldots, j)}=B^{(i, \ldots, j)} A^{(i, \ldots, j)}
$$

for the $\{i, \ldots, j\}$-inverses of the product of two singular matrices of $A$ and $B$. In these cases, people were naturally interested in establishing necessary and sufficient conditions for the reverse-order law to hold since Penrose defined in the 1950s the four matrix equations in (1.1). Different to the situations for nonsingular matrices, the reverse-order law in (1.1) have many forms due to the multiple choices of $\{i, \ldots, j\}$-inverses. The two well-known forms of $(1.3)$ are $(A B)^{\dagger}=B^{\dagger} A^{\dagger}$ and $(A B)^{(1)}=B^{(1)} A^{(1)}$; which were recognized and studied in the literature since the 1960 s; see, e.g., [6-23]. Because the $\{i, \ldots, j\}$-inverse of a matrix is defined from the four matrix equations in (1.1), it is straightforward to see that the reverseorder law in (1.3) holds if and only if some/all of the following four matrix equations:

$$
\begin{aligned}
& A B B^{(i, \ldots, j)} A^{(i, \ldots, j)} A B=A B, \\
& B^{(i, \ldots, j)} A^{(i, \ldots, j)} A B B^{(i, \ldots, j)} A^{(i, \ldots, j)}=B^{(i, \ldots, j)} A^{(i, \ldots, j)}, \\
& \left(A B B^{(i, \ldots, j)} A^{(i, \ldots, j)}\right)^{*}=A B B^{(i, \ldots, j)} A^{(i, \ldots, j)}, \\
& \left(B^{(i, \ldots, j)} A^{(i, \ldots, j)} A B\right)^{*}=B^{(i, \ldots, j)} A^{(i, \ldots, j)} A B,
\end{aligned}
$$

hold. Because $A^{(i, \ldots, j)}$ and $B^{(i, \ldots, j)}$ are not necessarily unique, (1.4)-(1.7) are four nonlinear matrix equations with respect to $A^{(i, \ldots, j)}$ and $B^{(i, \ldots, j)}$. Thus to characterize (1.3) is in fact to solve the four matrix equations in (1.4)-(1.7). This is really a challenging work on solving nonlinear matrix equations, and we need to make essential preparations in order to finish 
this algebraic task. In fact, reverse-order laws have belonged to the main objects of study in the theory of generalized inverses, which have leaded to some essential developments of the theory from the theoretical point of view. In particular, it greatly prompted establishments of many expansion formulas for calculating the ranks of matrices and their operations, and these rank formulas, as demonstrated below, now are widely used in matrix theory and applications. Note from (1.3) that the products $B^{(i, \ldots, j)} A^{(i, \ldots, j)}$ are building blocks for the constructions of the reverse-order laws and their variations. Hence it is necessary to study the constructions of the products $B^{(i, \ldots, j)} A^{(i, \ldots, j)}$ and their properties from the viewpoint of matrix-valued functions.

In order to reveal more deep and fundamental connections between both sides in (1.3), we consider in this paper some fundamental algebraic properties of the products on the right-hand side of (1.3). This paper studies a particular class of matrix rank optimization problems and establishes analytical formulas for calculating the maximum and minimum ranks of 64 possible products $B^{(i, \ldots, j)} A^{(i, \ldots, j)}$ on the right-hand side of (1.3), and use the rank formulas to investigates the performance of the products. Recall that the rank of matrix is a conceptual foundation in linear algebra and matrix theory, which is the most significant finite nonnegative integer in reflecting intrinsic properties of matrices. But it is really a technical task for people to establish thousands of influential and effective matrix rank formulas in the development of matrix theory and to use the formulas in the intuitive and rigorous investigations of matrices and applications. The intriguing connections between generalized inverses of matrices and rank formulas of matrices were recognized in the 1970s, and a seminal work on rank formulas for matrices and their generalized inverses was presented in [24]. The present author first introduced matrix rank formulas into the analysis of reverse-order laws of generalized inverses of matrix products in [25]. Over the last 40 years the theory of matrix ranks and its applications have grown into an active area of research in its own right, while a great wealth of literature was devoted to this subject and great successes were achieved during this approach. Now matrix rank formulas have become a magic weapon of simplifying and establishing various complicated matrix expressions and matrix equalities that involve generalized inverses of matrices.

\section{Preliminaries}

Recall a basic fact about matrix that $A=0$ if and only if $r(A)=0$. Thus, two matrices $A$ and $B$ of the same size are equal, namely, $A=B$, if and only if $r(A-B)=0$. Further, assume that $S_{1}$ and $S_{2}$ are two sets consisting of matrices of the same size. Then the following two assertions hold:

$$
\begin{aligned}
& S_{1} \cap S_{2} \neq \emptyset \quad \Leftrightarrow \quad \min _{A \in S_{1}, B \in S_{2}} r(A-B)=0 ; \\
& S_{1} \subseteq S_{2} \quad \Leftrightarrow \quad \max _{A \in S_{1}} \min _{B \in S_{2}} r(A-B)=0 .
\end{aligned}
$$

These implications provide a highly flexible framework for characterizing equalities of matrices via ranks of matrices. If certain formulas for calculating the rank of $A-B$ are derived, we can use the formulas to characterize relations between two matrices $A$ and $B$, and to obtain many valuable results. This method, called the matrix rank method, is available for studying various matrix expressions involving generalized inverses of matrices. Perhaps, no methods in linear algebra, as described above, is more elementary than the rank 
method in characterizing equalities of matrices. We see from (2.1) that the reverse-order law in (1.3) holds if and only if

$$
\min _{(A B)^{(i, \ldots, j)}, A^{(i, \ldots, j), B^{(i, \ldots, j)}}} r\left[(A B)^{(i, \ldots, j)}-B^{(i, \ldots, j)} A^{(i, \ldots, j)}\right]=0
$$

while the four equalities in (1.4)-(1.7) are equivalent to

$$
\begin{aligned}
& \min _{A^{(i, \ldots, j), B^{(i, \ldots, j)}}} r\left[A B B^{(i, \ldots, j)} A^{(i, \ldots, j)} A B-A B\right]=0, \\
& \min _{A^{(i, \ldots, j)}, B^{(i, \ldots, j)}} r\left[B^{(i, \ldots, j)} A^{(i, \ldots, j)} A B B^{(i, \ldots, j)} A^{(i, \ldots, j)}-B^{(i, \ldots, j)} A^{(i, \ldots, j)}\right]=0, \\
& \min _{A^{(i, \ldots, j)}, B^{(i, \ldots, j)}} r\left[\left(A B B^{(i, \ldots, j)} A^{(i, \ldots, j)}\right)^{*}-A B B^{(i, \ldots, j)} A^{(i, \ldots, j)}\right]=0, \\
& \min _{A^{(i, \ldots, j), B^{(i, \ldots, j)}}} r\left[\left(B^{(i, \ldots, j)} A^{(i, \ldots, j)} A B\right)^{*}-B^{(i, \ldots, j)} A^{(i, \ldots, j)} A B\right]=0 .
\end{aligned}
$$

Thus, if certain formulas for calculating the minimum ranks are given, we can derive necessary and sufficient conditions for (1.3) to hold from the rank formulas. This ideas was first introduced by the present author in [25] when characterizing reverse-order laws for the Moore-Penrose inverses of products of matrices, and have widely been used by many authors in the investigation of various types of reverse order law. To establish analytical formulas for calculating maximum and minimum ranks of matrix-valued functions is tricky and challenging work because of the integer property of the rank of matrix, and only pure algebraic methods are available to handle this kind of matrix rank optimization problems.

We next briefly introduce the mathematical foundations of our algebraic approaches to make the paper self-contained. The results in the following lemma are well known or follow from the definitions of $\{i, \ldots, j\}$-inverses of a matrix; see, e.g., $[5,11,12]$.

Lemma 1 Let $A \in \mathbb{C}^{m \times n}$. Then the following results hold.

(a) The general expressions of the last seven generalized inverses of $A$ in (1.2) can be written in the following parametric forms:

$$
\begin{aligned}
& A^{(1,3,4)}=A^{\dagger}+F_{A} V E_{A}, \\
& A^{(1,2,4)}=A^{\dagger}+A^{\dagger} A W E_{A}, \\
& A^{(1,2,3)}=A^{\dagger}+F_{A} V A A^{\dagger}, \\
& A^{(1,4)}=A^{\dagger}+W E_{A}, \\
& A^{(1,3)}=A^{\dagger}+F_{A} V, \\
& A^{(1,2)}=\left(A^{\dagger}+F_{A} V\right) A\left(A^{\dagger}+W E_{A}\right), \\
& A^{(1)}=A^{\dagger}+F_{A} V+W E_{A},
\end{aligned}
$$

where the two matrices $V, W \in \mathbb{C}^{n \times m}$ are arbitrary.

(b) The following matrix equalities hold:

$$
A A^{(1,3,4)}=A A^{(1,2,3)}=A A^{(1,3)}=A A^{\dagger},
$$




$$
\begin{aligned}
& A^{(1,3,4)} A=A^{(1,2,4)} A=A^{(1,4)} A=A^{\dagger} A, \\
& A A^{(1,2,4)}=A A^{(1,4)}=A A^{(1,2)}=A A^{(1)}=A A^{\dagger}+A W E_{A}, \\
& A^{(1,2,3)} A=A^{(1,3)} A=A^{(1,2)} A=A^{(1)} A=A^{\dagger} A+F_{A} V A,
\end{aligned}
$$

where the two matrices $V$ and $W$ are arbitrary.

(c) The following matrix set inclusions hold:

$$
\begin{aligned}
& A^{\dagger} \in\left\{A^{(1,3,4)}\right\} \subseteq\left\{A^{(1,4)}\right\} \subseteq\left\{A^{(1)}\right\}, \\
& A^{\dagger} \in\left\{A^{(1,3,4)}\right\} \subseteq\left\{A^{(1,3)}\right\} \subseteq\left\{A^{(1)}\right\}, \\
& A^{\dagger} \in\left\{A^{(1,2,4)}\right\} \subseteq\left\{A^{(1,4)}\right\} \subseteq\left\{A^{(1)}\right\}, \\
& A^{\dagger} \in\left\{A^{(1,2,4)}\right\} \subseteq\left\{A^{(1,2)}\right\} \subseteq\left\{A^{(1)}\right\}, \\
& A^{\dagger} \in\left\{A^{(1,2,3)}\right\} \subseteq\left\{A^{(1,3)}\right\} \subseteq\left\{A^{(1)}\right\}, \\
& A^{\dagger} \in\left\{A^{(1,2,3)}\right\} \subseteq\left\{A^{(1,2)}\right\} \subseteq\left\{A^{(1)}\right\} .
\end{aligned}
$$

(d) The following matrix set equalities hold:

$$
\begin{array}{ll}
\left\{\left(A^{(1,3,4)}\right)^{*}\right\}=\left\{\left(A^{*}\right)^{(1,3,4)}\right\}, & \left\{\left(A^{(1,2,4)}\right)^{*}\right\}=\left\{\left(A^{*}\right)^{(1,2,3)}\right\}, \\
\left\{\left(A^{(1,2,3)}\right)^{*}\right\}=\left\{\left(A^{*}\right)^{(1,2,4)}\right\}, & \left\{\left(A^{(1,4)}\right)^{*}\right\}=\left\{\left(A^{*}\right)^{(1,3)}\right\}, \\
\left\{\left(A^{(1,3)}\right)^{*}\right\}=\left\{\left(A^{*}\right)^{(1,4)}\right\}, & \left\{\left(A^{(1,2)}\right)^{*}\right\}=\left\{\left(A^{*}\right)^{(1,2)}\right\}, \\
\left\{\left(A^{(1)}\right)^{*}\right\}=\left\{\left(A^{*}\right)^{(1)}\right\} . &
\end{array}
$$

In order to establish and simplify various matrix equalities composed of generalized inverses of matrices, we need the following well-known rank formulas for matrices to make the paper self-contained.

Lemma 2 ([24]) Let $A \in \mathbb{C}^{m \times n}, B \in \mathbb{C}^{m \times k}$, and $C \in \mathbb{C}^{l \times n}$. Then

$$
\begin{aligned}
& r[A, B]=r(A)+r\left(E_{A} B\right)=r(B)+r\left(E_{B} A\right), \\
& r\left[\begin{array}{l}
A \\
C
\end{array}\right]=r(A)+r\left(C F_{A}\right)=r(C)+r\left(A F_{C}\right), \\
& r\left[\begin{array}{ll}
A & B \\
C & 0
\end{array}\right]=r(B)+r(C)+r\left(E_{B} A F_{C}\right) .
\end{aligned}
$$

Lemma 3 ([24]) Let $A \in \mathbb{C}^{m \times n}, B \in \mathbb{C}^{m \times k}$, and $C \in \mathbb{C}^{l \times n}$. Then the following results hold.

(a) $r[A, B]=r(A) \Leftrightarrow \mathscr{R}(B) \subseteq \mathscr{R}(A) \Leftrightarrow A A^{\dagger} B=B \Leftrightarrow E_{A} B=0$.

(b) $r\left[\begin{array}{l}A \\ C\end{array}\right]=r(A) \Leftrightarrow \mathscr{R}\left(C^{*}\right) \subseteq \mathscr{R}\left(A^{*}\right) \Leftrightarrow C A^{\dagger} A=C \Leftrightarrow C F_{A}=0$.

(c) $r[A, B]=r(A)+r(B) \Leftrightarrow \mathscr{R}(A) \cap \mathscr{R}(B)=\{0\} \Leftrightarrow \mathscr{R}\left[\left(E_{A} B\right)^{*}\right]=\mathscr{R}\left(B^{*}\right) \Leftrightarrow \mathscr{R}\left[\left(E_{B} A\right)^{*}\right]=$ $\mathscr{R}\left(A^{*}\right)$.

(d) $r\left[\begin{array}{l}A \\ { }_{C}\end{array}\right]=r(A)+r(C) \Leftrightarrow \mathscr{R}\left(A^{*}\right) \cap \mathscr{R}\left(C^{*}\right)=\{0\} \Leftrightarrow \mathscr{R}\left(C F_{A}\right)=\mathscr{R}(C) \Leftrightarrow \mathscr{R}\left(A F_{C}\right)=\mathscr{R}(A)$.

(e) $r\left[\begin{array}{ll}A & B \\ C & 0\end{array}\right]=r(A)+r(B)+r(C) \Leftrightarrow \mathscr{R}(A) \cap \mathscr{R}(B)=\{0\}$ and $\mathscr{R}\left(A^{*}\right) \cap \mathscr{R}\left(C^{*}\right)=\{0\}$. 
Lemma 4 ([24]) Let $A \in \mathbb{C}^{m \times n}$ and $B \in \mathbb{C}^{n \times p}$. Then the following results hold.

(a) The rank of $A B$ satisfies the expansion formulas

$$
\begin{aligned}
& r(A B)=r(A)+r(B)-n+r\left[\left(I_{n}-B B^{\dagger}\right)\left(I_{n}-A^{\dagger} A\right)\right], \\
& r(A B)=r(A)+r(B)-n+r\left[\left(I_{n}-B B^{(1)}\right)\left(I_{n}-A^{(1)} A\right)\right]
\end{aligned}
$$

for all $A^{(1)}$ and $B^{(1)}$.

(b) The rank of $A B$ satisfies the inequalities

$$
\begin{aligned}
& r(A B) \leq \min \{r(A), r(B)\} \leq \min \{m, n, p\}, \\
& r(A B) \geq r(A)+r(B)-r\left[A^{*}, B\right] \geq \max \{0, r(A)+r(B)-n\} .
\end{aligned}
$$

(c) The following statements are equivalent:

(i) $r(A B)=r(A)+r(B)-n$.

(ii) $\left(I_{n}-B B^{\dagger}\right)\left(I_{n}-A^{\dagger} A\right)=0$.

(iii) $\left(I_{n}-B B^{(1)}\right)\left(I_{n}-A^{(1)} A\right)=0$ for all $A^{(1)}$ and $B^{(1)}$.

(iv) $\mathscr{N}(A) \subseteq \mathscr{R}(B)$.

(v) $\mathscr{N}\left(B^{*}\right) \subseteq \mathscr{R}\left(A^{*}\right)$.

(d) The following statements are equivalent:

(i) $A B=0$.

(ii) $r(A)+r(B)=n-r\left[\left(I_{n}-B B^{\dagger}\right)\left(I_{n}-A^{\dagger} A\right)\right]$.

(iii) $\mathscr{R}(B) \subseteq \mathscr{N}(A)$.

(e) If $r(A B)=r(A)+r(B)-n$, then $r\left[A^{*}, B\right]=r(A)+r(B)-r(A B)=n$.

(f) If $A B=0$, then $r\left[A^{*}, B\right]=r(A)+r(B)$.

Lemma 5 Let $A \in \mathbb{C}^{m \times n}, M \in \mathbb{C}^{p \times n}$, and $B \in \mathbb{C}^{p \times q}$. Then the following rank equalities hold:

$$
\begin{aligned}
& r\left(B^{\dagger} M A^{\dagger}\right)=r\left(B^{*} M A^{\dagger}\right)=r\left(B^{\dagger} M A^{*}\right)=r\left(B^{*} M A^{*}\right), \\
& r\left(B B^{\dagger} M A^{\dagger} A\right)=r\left(B B^{*} M A^{\dagger}\right)=r\left(B B^{\dagger} M A^{*} A\right)=r\left(B B^{*} M A^{*} A\right)=r\left(B^{*} M A^{*}\right) .
\end{aligned}
$$

Because the rank of a matrix is a finite nonnegative integer, the ranks of matrix-valued functions are always bounded no matter what variable matrices in them are taken. In particular, the maximum and minimum ranks of a given matrix-valued function do always exist when variable matrices in the function run over certain feasible sets. In this case, it is desirable to establish analytical formulas for calculating the maximum and minimum ranks of the matrix-valued function over the feasible matrix sets, and use the maximum and minimum ranks to characterize some algebraic properties of the matrix-valued function. One of such optimization problems is to find the possible maximum and minimum ranks of the Schur complement $D-C A^{(i, \ldots, j)} B$ with respect to $A^{(i, \ldots, j)}$. In the past decades, some effective algebraic methods for dealing with ranks of matrices were developed, and many new matrix rank formulas were obtained. Especially, a comprehensive result on establishing rank formulas of $D-C A^{(i, \ldots, j)} B$ was given in [26]. As basic tools used in Section 3, we need the following matrix rank formulas. 
Lemma 6 ([26]) Let $A \in \mathbb{C}^{m \times n}, B \in \mathbb{C}^{m \times k}$, and $C \in \mathbb{C}^{l \times n}$. Then the following rank equalities hold:

$$
\begin{aligned}
& \max _{A^{(1,3,4)}} r\left(A^{(1,3,4)} B\right)=\min \left\{n+r\left(A^{*} B\right)-r(A), r(B)\right\}, \\
& \min _{A^{(1,3,4)}} r\left(A^{(1,3,4)} B\right)=r\left(A^{*} B\right) \\
& \max _{A^{(1,2,4)}} r\left(A^{(1,2,4)} B\right)=\max _{A^{(1,2,4)}} r\left(A A^{(1,2,4)} B\right)=\min \{r(A), r(B)\}, \\
& \min _{A^{(1,2,4)}} r\left(A^{(1,2,4)} B\right)=\min _{A^{(1,2,4)}} r\left(A A^{(1,2,4)} B\right)=r(A)+r(B)-r[A, B] \\
& \max _{A^{(1,2,3)}} r\left(A^{(1,2,3)} B\right)=\max _{A^{(1,2,3)}} r\left(A A^{(1,2,3)} B\right)=r\left(A^{*} B\right), \\
& \min _{A^{(1,2,3)}} r\left(A^{(1,2,3)} B\right)=\min _{A^{(1,2,3)}} r\left(A A^{(1,2,3)} B\right)=r\left(A^{*} B\right), \\
& \max _{A^{(1,4)}} r\left(A^{(1,4)} B\right)=\min \{n, r(B)\} \\
& \min _{A^{(1,4)}} r\left(A^{(1,4)} B\right)=r(A)+r(B)-r[A, B], \\
& \max _{A^{(1,3)}} r\left(A^{(1,3)} B\right)=\min \left\{n+r\left(A^{*} B\right)-r(A), r(B)\right\} \\
& \min _{A^{(1,3)}} r\left(A^{(1,3)} B\right)=r\left(A^{*} B\right) \\
& \max _{A^{(1,2)}} r\left(A^{(1,2)} B\right)=\max _{A^{(1,2)}} r\left(A A^{(1,2)} B\right)=\min \{r(A), r(B)\} \\
& \min _{A^{(1,2)}} r\left(A^{(1,2)} B\right)=\min _{A^{(1,2)}} r\left(A A^{(1,2)} B\right)=r(A)+r(B)-r[A, B], \\
& \max _{A^{(1)}} r\left(A^{(1)} B\right)=\min \{n, r(B)\} \\
& \min _{A^{(1)}} r\left(A^{(1)} B\right)=r(A)+r(B)-r[A, B], \\
&
\end{aligned}
$$

and

$$
\begin{aligned}
& \max _{A^{(1,3,4)}} r\left(C A^{(1,3,4)}\right)=\min \left\{m+r\left(C A^{*}\right)-r(A), r(C)\right\}, \\
& \min _{A^{(1,3,4)}} r\left(C A^{(1,3,4)}\right)=r\left(C A^{*}\right), \\
& \max _{A^{(1,2,4)}} r\left(C A^{(1,2,4)}\right)=\max _{A^{(1,2,4)}} r\left(C A^{(1,2,4)} A\right)=r\left(C A^{*}\right), \\
& \min _{A^{(1,2,4)}} r\left(C A^{(1,2,4)}\right)=\min _{A^{(1,2,4)}} r\left(C A^{(1,2,4)} A\right)=r\left(C A^{*}\right), \\
& \max _{A^{(1,2,3)}} r\left(C A^{(1,2,3)}\right)=\max _{A^{(1,2,3)}} r\left(C A^{(1,2,3)} A\right)=\min \{r(A), r(C)\}, \\
& \min _{A^{(1,2,3)}} r\left(C A^{(1,2,3)}\right)=\min _{A^{(1,2,3)}} r\left(C A^{(1,2,3)} A\right)=r(A)+r(C)-r\left[A^{*}, C^{*}\right], \\
& \max _{A^{(1,4)}} r\left(C A^{(1,4)}\right)=\min \left\{m+r\left(C A^{*}\right)-r(A), r(C)\right\}, \\
& \min _{A^{(1,4)}} r\left(C A^{(1,4)}\right)=r\left(C A^{*}\right),
\end{aligned}
$$




$$
\begin{aligned}
& \max _{A^{(1,3)}} r\left(C A^{(1,3)}\right)=\min \{m, r(C)\}, \\
& \min _{A^{(1,3)}} r\left(C A^{(1,3)}\right)=r(A)+r(C)-r\left[A^{*}, C^{*}\right], \\
& \max _{A^{(1,2)}} r\left(C A^{(1,2)}\right)=\max _{A^{(1,2)}} r\left(C A^{(1,2)} A\right)=\min \{r(A), r(C)\}, \\
& \min _{A^{(1,2)}} r\left(C A^{(1,2)}\right)=\min _{A^{(1,2)}} r\left(C A^{(1,2)} A\right)=r(A)+r(C)-r\left[A^{*}, C^{*}\right], \\
& \max _{A^{(1)}} r\left(C A^{(1)}\right)=\min \{m, r(C)\}, \\
& \min _{A^{(1)}} r\left(C A^{(1)}\right)=r(A)+r(C)-r\left[A^{*}, C^{*}\right] .
\end{aligned}
$$

Lemma 7 ([27]) Let

$$
q\left(X_{1}, X_{2}\right)=\left(A_{1}+B_{1} X_{1} C_{1}\right) D\left(A_{2}+B_{2} X_{2} C_{2}\right)
$$

be a quadratic matrix-valued function of appropriate sizes. Then the following rank equalities hold:

$$
\begin{aligned}
\max _{X_{1}, X_{2}} r\left[q\left(X_{1}, X_{2}\right)\right]= & \min \left\{r\left[A_{1} D A_{2}, A_{1} D B_{2}, B_{1}\right], r\left[\begin{array}{c}
A_{1} D A_{2} \\
C_{1} D A_{2} \\
C_{2}
\end{array}\right],\right. \\
& \left.r\left[\begin{array}{cc}
A_{1} D A_{2} & B_{1} \\
C_{2} & 0
\end{array}\right], r\left[\begin{array}{cc}
A_{1} D A_{2} & A_{1} D B_{2} \\
C_{1} D A_{2} & C_{1} D B_{2}
\end{array}\right]\right\}, \\
\min _{X_{1}, X_{2}} r\left[q\left(X_{1}, X_{2}\right)\right]= & r\left[\begin{array}{c}
A_{1} D A_{2} \\
C_{1} D A_{2} \\
C_{2}
\end{array}\right]+r\left[A_{1} D A_{2}, A_{1} D B_{2}, B_{1}\right]+\max \left\{s_{1}, s_{2}\right\},
\end{aligned}
$$

where

$$
\begin{aligned}
& s_{1}=r\left[\begin{array}{cc}
A_{1} D A_{2} & B_{1} \\
C_{2} & 0
\end{array}\right]-r\left[\begin{array}{ccc}
A_{1} D A_{2} & B_{1} & A_{1} D B_{2} \\
C_{2} & 0 & 0
\end{array}\right]-r\left[\begin{array}{cc}
A_{1} D A_{2} & B_{1} \\
C_{2} & 0 \\
C_{1} D A_{2} & 0
\end{array}\right], \\
& s_{2}=r\left[\begin{array}{ll}
A_{1} D A_{2} & A_{1} D B_{2} \\
C_{1} D A_{2} & C_{1} D B_{2}
\end{array}\right]-r\left[\begin{array}{ccc}
A_{1} D A_{2} & A_{1} D B_{2} & B_{1} \\
C_{1} D A_{2} & C_{1} D B_{2} & 0
\end{array}\right]-r\left[\begin{array}{cc}
A_{1} D A_{2} & A_{1} D B_{2} \\
C_{1} D A_{2} & C_{1} D B_{2} \\
C_{2} & 0
\end{array}\right] .
\end{aligned}
$$

Lemma 8 Let $\mathcal{S}$ and $\mathcal{T}$ be two sets consisting of appropriate sizes. Then the following results hold.

(a) The following implication holds:

$$
\mathcal{S} \supseteq \mathcal{T} \quad \Rightarrow \quad P S Q \supseteq P \mathcal{T} Q
$$

(b) The following rank inequalities hold:

$$
\max _{X \in \mathcal{S}} r(X) \geq \max _{Y \in P \mathcal{S} Q} r(Y), \quad \min _{X \in \mathcal{S}} r(X) \geq \min _{Y \in P \mathcal{S} Q} r(Y) .
$$


(c) The following implications hold:

$$
\mathcal{S} \supseteq \mathcal{T} \Rightarrow \max _{X \in \mathcal{S}} r(X) \geq \max _{Y \in \mathcal{T}} r(Y) \text { and } \min _{X \in \mathcal{S}} r(X) \leq \min _{Y \in \mathcal{T}} r(Y)
$$

Proof Result (a) is obvious from the fact that the matrix equality $S=T$ implies the matrix equality $P S Q=P T Q$. The two rank inequalities in (2.69) follow from the well-known rank inequality $r(S) \geq r(P S Q)$. The implications in (2.70) are obvious.

Lemma 9 Let $A \in \mathbb{C}^{m \times n}$. Then the following rank inequalities hold:

$$
\begin{aligned}
& r(A)=r\left(A^{\dagger}\right) \leq \max _{A^{(1,3,4)}} r\left(A^{(1,3,4)}\right) \leq \max _{A^{(1,4)}} r\left(A^{(1,4)}\right) \leq \max _{A^{(1)}} r\left(A^{(1)}\right)=\min \{m, n\}, \\
& r(A)=r\left(A^{\dagger}\right) \leq \max _{A^{(1,3,4)}} r\left(A^{(1,3,4)}\right) \leq \max _{A^{(1,3)}} r\left(A^{(1,3)}\right) \leq \max _{A^{(1)}} r\left(A^{(1)}\right)=\min \{m, n\}, \\
& r(A)=r\left(A^{\dagger}\right) \leq \max _{A^{(1,2,4)}} r\left(A^{(1,2,4)}\right) \leq \max _{A^{(1,4)}} r\left(A^{(1,4)}\right) \leq \max _{A^{(1)}} r\left(A^{(1)}\right)=\min \{m, n\}, \\
& r(A)=r\left(A^{\dagger}\right) \leq \max _{A^{(1,2,4)}} r\left(A^{(1,2,4)}\right) \leq \max _{A^{(1,2)}} r\left(A^{(1,2)}\right) \leq \max _{A^{(1)}} r\left(A^{(1)}\right)=\min \{m, n\}, \\
& r(A)=r\left(A^{\dagger}\right) \leq \max _{A^{(1,2,3)}} r\left(A^{(1,2,3)}\right) \leq \max _{A^{(1,3)}} r\left(A^{(1,3)}\right) \leq \max _{A^{(1)}} r\left(A^{(1)}\right)=\min \{m, n\}, \\
& r(A)=r\left(A^{\dagger}\right) \leq \max _{A^{(1,2,3)}} r\left(A^{(1,2,3)}\right) \leq \max _{A^{(1,2)}} r\left(A^{(1,2)}\right) \leq \max _{A^{(1)}} r\left(A^{(1)}\right)=\min \{m, n\} .
\end{aligned}
$$

Proof The proof follows from (2.19)-(2.24) and (2.50).

\section{Main results}

Let $A \in \mathbb{C}^{m \times n}$ and $B \in \mathbb{C}^{n \times p}$. In order to characterize performances of the product $B^{(i, \ldots, j)} A^{(i, \ldots, j)}$, we first give their parametric forms of the products

$$
B^{(i, \ldots, j)} A^{(i, \ldots, j)}
$$

for the eight commonly used generalized inverses $A^{(i, \ldots, j)}$ and $B^{(i, \ldots, j)}$, respectively. From (2.8)-(2.14), the 63 parametric expressions of (3.1) corresponding to $A^{(i, \ldots, j)}$ and $B^{(i, \ldots, j)}$ can be written as

$$
\begin{aligned}
& B^{\dagger} A^{(1,3,4)}=B^{\dagger} A^{\dagger}+B^{\dagger} F_{A} W E_{A}, \\
& B^{\dagger} A^{(1,2,4)}=B^{\dagger} A^{\dagger}+B^{\dagger} A^{\dagger} A W E_{A}, \\
& B^{\dagger} A^{(1,2,3)}=B^{\dagger} A^{\dagger}+B^{\dagger} F_{A} W A A^{\dagger}, \\
& B^{\dagger} A^{(1,4)}=B^{\dagger} A^{\dagger}+B^{\dagger} W E_{A}, \\
& B^{\dagger} A^{(1,3)}=B^{\dagger} A^{\dagger}+B^{\dagger} F_{A} W, \\
& B^{\dagger} A^{(1,2)}=\left(B^{\dagger} A^{\dagger}+B^{\dagger} F_{A} W_{1}\right) A\left(A^{\dagger}+W_{2} E_{A}\right), \\
& B^{\dagger} A^{(1)}=B^{\dagger} A^{\dagger}+B^{\dagger} F_{A} W_{1}+B^{\dagger} W_{2} E_{A}, \\
& B^{(1,3,4)} A^{\dagger}=B^{\dagger} A^{\dagger}+F_{B} V E_{B} A^{\dagger}, \\
& B^{(1,3,4)} A^{(1,3,4)}=\left(B^{\dagger}+F_{B} V E_{B}\right)\left(A^{\dagger}+F_{A} W E_{A}\right),
\end{aligned}
$$




$$
\begin{aligned}
& B^{(1,3,4)} A^{(1,2,4)}=\left(B^{\dagger}+F_{B} V E_{B}\right)\left(A^{\dagger}+A^{\dagger} A W E_{A}\right), \\
& B^{(1,3,4)} A^{(1,2,3)}=\left(B^{\dagger}+F_{B} V E_{B}\right)\left(A^{\dagger}+F_{A} W A A^{\dagger}\right), \\
& B^{(1,3,4)} A^{(1,4)}=\left(B^{\dagger}+F_{B} V E_{B}\right)\left(A^{\dagger}+W E_{A}\right), \\
& B^{(1,3,4)} A^{(1,3)}=\left(B^{\dagger}+F_{B} V E_{B}\right)\left(A^{\dagger}+F_{A} W\right), \\
& B^{(1,3,4)} A^{(1,2)}=\left(B^{\dagger}+F_{B} V E_{B}\right)\left(A^{\dagger}+F_{A} W_{1}\right) A\left(A^{\dagger}+W_{2} E_{A}\right) \text {, } \\
& B^{(1,3,4)} A^{(1)}=\left(B^{\dagger}+F_{B} V E_{B}\right)\left(A^{\dagger}+F_{A} W_{1}+W_{2} E_{A}\right), \\
& B^{(1,2,4)} A^{\dagger}=B^{\dagger} A^{\dagger}+B^{\dagger} B V E_{B} A^{\dagger} \text {, } \\
& B^{(1,2,4)} A^{(1,3,4)}=\left(B^{\dagger}+B^{\dagger} B V E_{B}\right)\left(A^{\dagger}+F_{A} W E_{A}\right), \\
& B^{(1,2,4)} A^{(1,2,4)}=\left(B^{\dagger}+B^{\dagger} B V E_{B}\right)\left(A^{\dagger}+A^{\dagger} A W E_{A}\right), \\
& B^{(1,2,4)} A^{(1,2,3)}=\left(B^{\dagger}+B^{\dagger} B V E_{B}\right)\left(A^{\dagger}+F_{A} W A A^{\dagger}\right), \\
& B^{(1,2,4)} A^{(1,4)}=\left(B^{\dagger}+B^{\dagger} B V E_{B}\right)\left(A^{\dagger}+W E_{A}\right), \\
& B^{(1,2,4)} A^{(1,3)}=\left(B^{\dagger}+B^{\dagger} B V E_{B}\right)\left(A^{\dagger}+F_{A} W\right), \\
& B^{(1,2,4)} A^{(1,2)}=\left(B^{\dagger}+B^{\dagger} B V E_{B}\right)\left(A^{\dagger}+F_{A} W_{1}\right) A\left(A^{\dagger}+W_{2} E_{A}\right), \\
& B^{(1,2,4)} A^{(1)}=\left(B^{\dagger}+B^{\dagger} B V E_{B}\right)\left(A^{\dagger}+F_{A} W_{1}+W_{2} E_{A}\right), \\
& B^{(1,2,3)} A^{\dagger}=B^{\dagger} A^{\dagger}+F_{B} V B B^{\dagger} A^{\dagger} \text {, } \\
& B^{(1,2,3)} A^{(1,3,4)}=\left(B^{\dagger}+F_{B} V B B^{\dagger}\right)\left(A^{\dagger}+F_{A} W E_{A}\right), \\
& B^{(1,2,3)} A^{(1,2,4)}=\left(B^{\dagger}+F_{B} V B B^{\dagger}\right)\left(A^{\dagger}+A^{\dagger} A W E_{A}\right), \\
& B^{(1,2,3)} A^{(1,2,3)}=\left(B^{\dagger}+F_{B} V B B^{\dagger}\right)\left(A^{\dagger}+F_{A} W A A^{\dagger}\right), \\
& B^{(1,2,3)} A^{(1,4)}=\left(B^{\dagger}+F_{B} V B B^{\dagger}\right)\left(A^{\dagger}+W E_{A}\right), \\
& B^{(1,2,3)} A^{(1,3)}=\left(B^{\dagger}+F_{B} V B B^{\dagger}\right)\left(A^{\dagger}+F_{A} W\right), \\
& B^{(1,2,3)} A^{(1,2)}=\left(B^{\dagger}+F_{B} V B B^{\dagger}\right)\left(A^{\dagger}+F_{A} W_{1}\right) A\left(A^{\dagger}+W_{2} E_{A}\right), \\
& B^{(1,2,3)} A^{(1)}=\left(B^{\dagger}+F_{B} V B B^{\dagger}\right)\left(A^{\dagger}+F_{A} W_{1}+W_{2} E_{A}\right), \\
& B^{(1,4)} A^{\dagger}=B^{\dagger} A^{\dagger}+V E_{B} A^{\dagger} \text {, } \\
& B^{(1,4)} A^{(1,3,4)}=\left(B^{\dagger}+V E_{B}\right)\left(A^{\dagger}+F_{A} W E_{A}\right), \\
& B^{(1,4)} A^{(1,2,4)}=\left(B^{\dagger}+V E_{B}\right)\left(A^{\dagger}+A^{\dagger} A W E_{A}\right), \\
& B^{(1,4)} A^{(1,2,3)}=\left(B^{\dagger}+V E_{B}\right)\left(A^{\dagger}+F_{A} W A A^{\dagger}\right), \\
& B^{(1,4)} A^{(1,4)}=\left(B^{\dagger}+V E_{B}\right)\left(A^{\dagger}+W E_{A}\right), \\
& B^{(1,4)} A^{(1,3)}=\left(B^{\dagger}+V E_{B}\right)\left(A^{\dagger}+F_{A} W\right), \\
& B^{(1,4)} A^{(1,2)}=\left(B^{\dagger}+V E_{B}\right)\left(A^{\dagger}+F_{A} W_{1}\right) A\left(A^{\dagger}+W_{2} E_{A}\right), \\
& B^{(1,4)} A^{(1)}=\left(B^{\dagger}+V E_{B}\right)\left(A^{\dagger}+F_{A} W_{1}+W_{2} E_{A}\right), \\
& B^{(1,3)} A^{\dagger}=B^{\dagger} A^{\dagger}+F_{B} V A^{\dagger} \text {, } \\
& B^{(1,3)} A^{(1,3,4)}=\left(B^{\dagger}+F_{B} V\right)\left(A^{\dagger}+F_{A} W E_{A}\right), \\
& B^{(1,3)} A^{(1,2,4)}=\left(B^{\dagger}+F_{B} V\right)\left(A^{\dagger}+A^{\dagger} A W E_{A}\right),
\end{aligned}
$$




$$
\begin{aligned}
& B^{(1,3)} A^{(1,2,3)}=\left(B^{\dagger}+F_{B} V\right)\left(A^{\dagger}+F_{A} W A A^{\dagger}\right), \\
& B^{(1,3)} A^{(1,4)}=\left(B^{\dagger}+F_{B} V\right)\left(A^{\dagger}+W E_{A}\right), \\
& B^{(1,3)} A^{(1,3)}=\left(B^{\dagger}+F_{B} V\right)\left(A^{\dagger}+F_{A} W\right), \\
& B^{(1,3)} A^{(1,2)}=\left(B^{\dagger}+F_{B} V\right)\left(A^{\dagger}+F_{A} W_{1}\right) A\left(A^{\dagger}+W_{2} E_{A}\right), \\
& B^{(1,3)} A^{(1)}=\left(B^{\dagger}+F_{B} V\right)\left(A^{\dagger}+F_{A} W_{1}+W_{2} E_{A}\right), \\
& B^{(1,2)} A^{\dagger}=\left(B^{\dagger}+F_{B} V_{1}\right) B\left(B^{\dagger} A^{\dagger}+V_{2} E_{B} A^{\dagger}\right), \\
& B^{(1,2)} A^{(1,3,4)}=\left(B^{\dagger}+F_{B} V_{1}\right) B\left(B^{\dagger}+V_{2} E_{B}\right)\left(A^{\dagger}+F_{A} W E_{A}\right), \\
& B^{(1,2)} A^{(1,2,4)}=\left(B^{\dagger}+F_{B} V_{1}\right) B\left(B^{\dagger}+V_{2} E_{B}\right)\left(A^{\dagger}+A^{\dagger} A W E_{A}\right), \\
& B^{(1,2)} A^{(1,2,3)}=\left(B^{\dagger}+F_{B} V_{1}\right) B\left(B^{\dagger}+V_{2} E_{B}\right)\left(A^{\dagger}+F_{A} W A A^{\dagger}\right), \\
& B^{(1,2)} A^{(1,4)}=\left(B^{\dagger}+F_{B} V_{1}\right) B\left(B^{\dagger}+V_{2} E_{B}\right)\left(A^{\dagger}+W E_{A}\right), \\
& B^{(1,2)} A^{(1,3)}=\left(B^{\dagger}+F_{B} V_{1}\right) B\left(B^{\dagger}+V_{2} E_{B}\right)\left(A^{\dagger}+F_{A} W\right), \\
& B^{(1,2)} A^{(1,2)}=\left(B^{\dagger}+F_{B} V_{1}\right) B\left(B^{\dagger}+V_{2} E_{B}\right)\left(A^{\dagger}+F_{A} W_{1}\right) A\left(A^{\dagger}+W_{2} E_{A}\right), \\
& B^{(1,2)} A^{(1)}=\left(B^{\dagger}+F_{B} V_{1}\right) B\left(B^{\dagger}+V_{2} E_{B}\right)\left(A^{\dagger}+F_{A} W_{1}+W_{2} E_{A}\right), \\
& B^{(1)} A^{\dagger}=B^{\dagger} A^{\dagger}+F_{B} V_{1} A^{\dagger}+V_{2} E_{B} A^{\dagger}, \\
& B^{(1)} A^{(1,3,4)}=\left(B^{\dagger}+F_{B} V_{1}+V_{2} E_{B}\right)\left(A^{\dagger}+F_{A} W E_{A}\right), \\
& B^{(1)} A^{(1,2,4)}=\left(B^{\dagger}+F_{B} V_{1}+V_{2} E_{B}\right)\left(A^{\dagger}+A^{\dagger} A W E_{A}\right), \\
& B^{(1)} A^{(1,2,3)}=\left(B^{\dagger}+F_{B} V_{1}+V_{2} E_{B}\right)\left(A^{\dagger}+F_{A} W A A^{\dagger}\right), \\
& B^{(1)} A^{(1,4)}=\left(B^{\dagger}+F_{B} V_{1}+V_{2} E_{B}\right)\left(A^{\dagger}+W E_{A}\right), \\
& B^{(1)} A^{(1,3)}=\left(B^{\dagger}+F_{B} V_{1}+V_{2} E_{B}\right)\left(A^{\dagger}+F_{A} W\right), \\
& B^{(1)} A^{(1,2)}=\left(B^{\dagger}+F_{B} V_{1}+V_{2} E_{B}\right)\left(A^{\dagger}+F_{A} W_{1}\right) A\left(A^{\dagger}+W_{2} E_{A}\right), \\
& B^{(1)} A^{(1)}=\left(B^{\dagger}+F_{B} V_{1}+V_{2} E_{B}\right)\left(A^{\dagger}+F_{A} W_{1}+W_{2} E_{A}\right), \\
&
\end{aligned}
$$

where $V, V_{1}, V_{2}, W, W_{1}, W_{2}$ are arbitrary matrices of appropriate sizes. From (3.2)-(3.64), we further obtain the following results on the analytical expressions of

$$
\begin{array}{ll}
B B^{(i, \ldots, j)} A^{(i, \ldots, j)} A, & A^{(i, \ldots, j)} A B B^{(i, \ldots, j)}, \\
A B B^{(i, \ldots, j)} A^{(i, \ldots, j)}, & B^{(i, \ldots, j)} A^{(i, \ldots, j)} A B,
\end{array}
$$

(I) The products $B B^{(i, \ldots, j)} A^{(i, \ldots, j)} A$ can be written as the following four groups of expression:

$$
\begin{aligned}
B B^{\dagger} & A^{(1,3,4)} A \\
& =B B^{\dagger} A^{(1,2,4)} A=B B^{\dagger} A^{(1,4)} A=B B^{(1,3,4)} A^{\dagger} A \\
& =B B^{(1,3,4)} A^{(1,3,4)} A=B B^{(1,3,4)} A^{(1,2,4)} A=B B^{(1,3,4)} A^{(1,4)} A=B B^{(1,2,3)} A^{\dagger} A \\
& =B B^{(1,2,3)} A^{(1,3,4)} A=B B^{(1,2,3)} A^{(1,2,4)} A=B B^{(1,2,3)} A^{(1,4)} A=B B^{(1,3)} A^{\dagger} A \\
& =B B^{(1,3)} A^{(1,3,4)} A=B B^{(1,3)} A^{(1,2,4)} A=B B^{(1,3)} A^{(1,4)} A \\
& =B B^{\dagger} A^{\dagger} A,
\end{aligned}
$$




$$
\begin{aligned}
& B B^{\dagger} A^{(1,2,3)} A \\
& =B B^{\dagger} A^{(1,3)} A=B B^{\dagger} A^{(1,2)} A=B B^{\dagger} A^{(1)} A \\
& =B B^{(1,3,4)} A^{(1,2,3)} A=B B^{(1,3,4)} A^{(1,3)} A=B B^{(1,3,4)} A^{(1,2)} A=B B^{(1,3,4)} A^{(1)} A \\
& =B B^{(1,2,3)} A^{(1,2,3)} A=B B^{(1,2,3)} A^{(1,3)} A=B B^{(1,2,3)} A^{(1,2)} A=B B^{(1,2,3)} A^{(1)} A \\
& =B B^{(1,3)} A^{(1,2,3)} A=B B^{(1,3)} A^{(1,3)} A=B B^{(1,3)} A^{(1,2)} A=B B^{(1,3)} A^{(1)} A \\
& =B B^{\dagger} A^{\dagger} A+B B^{\dagger} F_{A} W A, \\
& B B^{(1,2,4)} A^{\dagger} A \\
& =B B^{(1,2,4)} A^{(1,3,4)} A=B B^{(1,2,4)} A^{(1,2,4)} A=B B^{(1,2,4)} A^{(1,4)} A \\
& =B B^{(1,4)} A^{\dagger} A=B B^{(1,4)} A^{(1,3,4)} A=B B^{(1,4)} A^{(1,2,4)} A=B B^{(1,4)} A^{(1,4)} A \\
& =B B^{(1,2)} A^{\dagger} A=B B^{(1,2)} A^{(1,3,4)} A=B B^{(1,2)} A^{(1,2,4)} A=B B^{(1,2)} A^{(1,4)} A \\
& =B B^{(1)} A^{\dagger} A=B B^{(1)} A^{(1,3,4)} A=B B^{(1)} A^{(1,2,4)} A=B B^{(1)} A^{(1,4)} A \\
& =B B^{\dagger} A^{\dagger} A+B V E_{B} A^{\dagger} A \text {, } \\
& B B^{(1,2,4)} A^{(1,2,3)} A \\
& =B B^{(1,2,4)} A^{(1,3)} A=B B^{(1,2,4)} A^{(1,2)} A=B B^{(1,2,4)} A^{(1)} A \\
& =B B^{(1,4)} A^{(1,2,3)} A=B B^{(1,4)} A^{(1,3)} A=B B^{(1,4)} A^{(1,2)} A=B B^{(1,4)} A^{(1)} A \\
& =B B^{(1,2)} A^{(1,2,3)} A=B B^{(1,2)} A^{(1,3)} A=B B^{(1,2)} A^{(1,2)} A=B B^{(1)} A^{(1)} A \\
& =B B^{(1)} A^{(1,2,3)} A=B B^{(1)} A^{(1,3)} A=B B^{(1)} A^{(1,2)} A=B B^{(1)} A^{(1)} A \\
& =\left(B B^{\dagger}+B V E_{B}\right)\left(A^{\dagger} A+F_{A} W A\right),
\end{aligned}
$$

where $V$ and $W$ are variable matrices of appropriate sizes.

(II) The products $A^{(i, \ldots, j)} A B B^{(i, \ldots, j)}$ can be written as the following four groups of expression:

$$
\begin{aligned}
A^{(1,3,4)} A B B^{\dagger} & \\
= & A^{(1,2,4)} A B B^{\dagger}=A^{(1,4)} A B B^{\dagger}=B B^{(1,3,4)} A^{\dagger} A \\
& =A^{(1,3,4)} A B B^{(1,3,4)}=A^{(1,2,4)} A B B^{(1,3,4)}=A^{(1,4)} A B B^{(1,3,4)}=A^{\dagger} A B B^{(1,2,3)} \\
& =A^{(1,3,4)} A B B^{(1,2,3)}=A^{(1,2,4)} A B B^{(1,2,3)}=A^{(1,4)} A B B^{(1,2,3)}=A^{\dagger} A B B^{(1,3)} \\
& =A^{(1,3)} A B B^{(1,3)}=A^{(1,2,4)} A B B^{(1,3)}=A^{(1,4)} A B B^{(1,3)} \\
& =A^{\dagger} A B B^{\dagger}, \\
A^{(1,2,3)} A B B^{\dagger} & \\
& =A^{(1,3)} A B B^{\dagger}=A^{(1,2)} A B B^{\dagger}=A^{(1)} A B B^{\dagger} \\
& =A^{(1,2,3)} A B B^{(1,3,4)}=A^{(1,3)} A B B^{(1,3,4)}=A^{(1,2)} A B B^{(1,3,4)}=A^{(1)} A B B^{(1,3,4)} \\
& =A^{(1,2,3)} A B B^{(1,2,3)}=A^{(1,3)} A B B^{(1,2,3)}=A^{(1,2)} A B B^{(1,2,3)}=A^{(1)} A B B^{(1,2,3)} \\
& =A^{(1,2,3)} A B B^{(1,3)}=A^{(1,3)} A B B^{(1,3)}=A^{(1,2)} A B B^{(1,3)}=A^{(1)} A B B^{(1,3)} \\
& =A^{\dagger} A B B^{\dagger}+F_{A} W A B B^{\dagger},
\end{aligned}
$$




$$
\begin{aligned}
A^{\dagger} & A B B^{(1,2,4)} \\
& =A^{(1,3,4)} A B B^{(1,2,4)}=A^{(1,2,4)} A B B^{(1,2,4)}=A^{(1,4)} A B B^{(1,2,4)} \\
& =A^{\dagger} A B B^{(1,4)}=A^{(1,3,4)} A B B^{(1,4)}=A^{(1,2,4)} A B B^{(1,4)}=A^{(1,4)} A B B^{(1,4)} \\
& =A^{\dagger} A B B^{(1,2)}=A^{(1,3,4)} A B B^{(1,2)}=A^{(1,2,4)} A B B^{(1,2)}=A^{(1,4)} A B B^{(1,2)} \\
& =A^{\dagger} A B B^{(1)}=A^{(1,3,4)} A B B^{(1)}=A^{(1,2,4)} A B B^{(1)}=A^{(1,4)} A B B^{(1)} \\
& =A^{\dagger} A B B^{\dagger}+A^{\dagger} A B V E_{B}, \\
A^{(1,2,3)} & A B B^{(1,2,4)} \\
& =A^{(1,3)} A B B^{(1,2,4)}=A^{(1,2)} A B B^{(1,2,4)}=A^{(1)} A B B^{(1,2,4)} \\
& =A^{(1,2,3)} A B B^{(1,4)}=A^{(1,3)} A B B^{(1,4)}=A^{(1,2)} A B B^{(1,4)}=A^{(1)} A B B^{(1,4)} \\
& =A^{(1,2,3)} A B B^{(1,2)}=A^{(1,3)} A B B^{(1,2)}=A^{(1,2)} A B B^{(1,2)}=A^{(1)} A B B^{(1,2)} \\
& =A^{(1,2,3)} A B B^{(1)}=A^{(1,3)} A B B^{(1)}=A^{(1,2)} A B B^{(1)}=A^{(1)} A B B^{(1)} \\
& =\left(A^{\dagger} A+F_{A} W A\right)\left(B B^{\dagger}+B V E_{B}\right),
\end{aligned}
$$

where $V$ and $W$ are variable matrices of appropriate sizes.

(III) The products $A B B^{(i, \ldots, j)} A^{(i, \ldots, j)}$ and $B^{(i, \ldots, j)} A^{(i, \ldots, j)} A B$ can be written as the following groups of expression:

$$
\begin{aligned}
A B B^{(1,3,4)} A^{\dagger} & =A B B^{(1,2,3)} A^{\dagger}=A B B^{(1,3)} A^{\dagger}=A B B^{\dagger} A^{\dagger} \\
A B B^{\dagger} A^{(1,3,4)}= & A B B^{(1,3,4)} A^{(1,3,4)}=A B B^{(1,2,3)} A^{(1,3,4)}=A B B^{(1,3)} A^{(1,3,4)} \\
& =A B B^{\dagger} A^{\dagger}+A B B^{\dagger} F_{A} W E_{A}, \\
A B B^{\dagger} A^{(1,2,4)}= & A B B^{(1,3,4)} A^{(1,2,4)}=A B B^{(1,2,3)} A^{(1,2,4)}=A B B^{(1,3)} A^{(1,2,4)} \\
& =A B B^{\dagger} A^{\dagger}+A B B^{\dagger} A^{\dagger} A W E_{A}, \\
A B B^{\dagger} A^{(1,2,3)}= & A B B^{(1,3,4)} A^{(1,2,3)}=A B B^{(1,2,3)} A^{(1,2,3)}=A B B^{(1,3)} A^{(1,2,3)} \\
& =A B B^{\dagger} A^{\dagger}+A B B^{\dagger} F_{A} W A A^{\dagger}, \\
A B B^{\dagger} A^{(1,4)}= & A B B^{(1,3,4)} A^{(1,4)}=A B B^{(1,2,3)} A^{(1,4)}=A B B^{(1,3)} A^{(1,4)} \\
= & A B B^{\dagger} A^{\dagger}+A B B^{\dagger} W E_{A}, \\
A B B^{\dagger} A^{(1,3)}= & A B B^{(1,3,4)} A^{(1,3)}=A B B^{(1,2,3)} A^{(1,3)}=A B B^{(1,3)} A^{(1,3)} \\
= & A B B^{\dagger} A^{\dagger}+A B B^{\dagger} F_{A} W, \\
A B B^{(1,2,4)} A^{\dagger} & =A B B^{(1,4)} A^{\dagger}=A B B^{(1,2)} A^{\dagger}=A B B^{(1)} A^{\dagger} \\
& =A B B^{\dagger} A^{\dagger}+A B V E_{B} A^{\dagger}, \\
A B B^{\dagger} A^{(1)}= & A B B^{(1,3,4)} A^{(1)}=A B B^{(1,2,3)} A^{(1)}=A B B^{(1,3)} A^{(1)} \\
= & A B B^{\dagger} A^{\dagger}+A B B^{\dagger} F_{A} W_{1}+A B B^{\dagger} W_{2} E_{A}, \\
A^{(1,2)}= & A B B^{(1,3,4)} A^{(1,2)}=A B B^{(1,2,3)} A^{(1,2)}=A B B^{(1,3)} A^{(1,2)} \\
& \left.A B B^{\dagger} A^{\dagger}+A B B^{\dagger} F_{A} W_{1}\right) A\left(A^{\dagger}+W_{2} E_{A}\right),
\end{aligned}
$$




$$
\begin{aligned}
A B B^{(1,2,4)} A^{(1,3,4)}= & A B B^{(1,4)} A^{(1,3,4)}=A B B^{(1,2)} A^{(1,3,4)}=A B B^{(1)} A^{(1,3,4)} \\
& =\left(A B B^{\dagger}+A B V E_{B}\right)\left(A^{\dagger}+F_{A} W E_{A}\right), \\
A B B^{(1,2,4)} A^{(1,2,4)} & =A B B^{(1,4)} A^{(1,2,4)}=A B B^{(1,2)} A^{(1,2,4)}=A B B^{(1)} A^{(1,2,4)} \\
& =\left(A B B^{\dagger}+A B V E_{B}\right)\left(A^{\dagger}+A^{\dagger} A W E_{A}\right), \\
A B B^{(1,2,4)} A^{(1,2,3)} & =A B B^{(1,4)} A^{(1,2,3)}=A B B^{(1,2)} A^{(1,2,3)}=A B B^{(1)} A^{(1,2,3)} \\
& =\left(A B B^{\dagger}+A B V E_{B}\right)\left(A^{\dagger}+F_{A} W A A^{\dagger}\right), \\
A B B^{(1,2,4)} A^{(1,4)}= & A B B^{(1,4)} A^{(1,4)}=A B B^{(1,2)} A^{(1,4)}=A B B^{(1)} A^{(1,4)} \\
= & \left(A B B^{\dagger}+A B V E_{B}\right)\left(A^{\dagger}+W_{A}\right), \\
A B B^{(1,2,4)} A^{(1,3)}= & A B B^{(1,4)} A^{(1,3)}=A B B^{(1,2)} A^{(1,3)}=A B B^{(1)} A^{(1,3)} \\
= & \left(A B B^{\dagger}+A B V E_{B}\right)\left(A^{\dagger}+F_{A} W\right), \\
A B B^{(1,2,4)} A^{(1,2)}= & A B B^{(1,4)} A^{(1,2)}=A B B^{(1,2)} A^{(1,2)}=A B B^{(1)} A^{(1,2)} \\
= & \left(A B B^{\dagger}+A B V E_{B}\right)\left(A^{\dagger}+F_{A} W{ }_{1}\right) A\left(A^{\dagger}+W_{2} E_{A}\right), \\
A B B^{(1,2,4)} A^{(1)}= & A B B^{(1,4)} A^{(1)}=A B B^{(1,2)} A^{(1)}=A B B^{(1)} A^{(1)} \\
= & \left(A B B^{\dagger}+A B V E_{B}\right)\left(A^{\dagger}+F_{A} W_{1}+W_{2} E_{A}\right),
\end{aligned}
$$

and

$$
\begin{aligned}
& B^{\dagger} A^{(1,3,4)} A B=B^{\dagger} A^{(1,2,4)} A B=B^{\dagger} A^{(1,4)} A B=B^{\dagger} A^{\dagger} A B \\
& B^{(1,3,4)} A^{\dagger} A B=B^{(1,3,4)} A^{(1,3,4)} A B=B^{(1,3,4)} A^{(1,2,4)} A B=B^{(1,3,4)} A^{(1,4)} A B \\
&=B^{\dagger} A^{\dagger} A B+F_{B} V E_{B} A^{\dagger} A B, \\
& B^{(1,2,4)} A^{\dagger} A B=B^{(1,2,4)} A^{(1,3,4)} A B=B^{(1,2,4)} A^{(1,2,4)} A B=B^{(1,2,4)} A^{(1,4)} A B \\
&=B^{\dagger} A^{\dagger} A B+B^{\dagger} B V E_{B} A^{\dagger} A B, \\
& B^{(1,2,3)} A^{\dagger} A B=B^{(1,2,3)} A^{(1,3,4)} A B=B^{(1,2,3)} A^{(1,2,4)} A B=B^{(1,2,3)} A^{(1,4)} A B \\
&=B^{\dagger} A^{\dagger} A B+F_{B} V B B^{\dagger} A^{\dagger} A B, \\
& B^{(1,4)} A^{\dagger} A B= B^{(1,4)} A^{(1,3,4)} A B=B^{(1,4)} A^{(1,2,4)} A B=B^{(1,4)} A^{(1,4)} A B \\
&= B^{\dagger} A^{\dagger} A B+V E_{B} A^{\dagger} A B, \\
& B^{(1,3)} A^{\dagger} A B= B^{(1,3)} A^{(1,3,4)} A B=B^{(1,3)} A^{(1,2,4)} A B=B^{(1,3)} A^{(1,4)} A B \\
&= B^{\dagger} A^{\dagger} A B+F_{B} V A^{\dagger} A B, \\
& B^{\dagger} A^{(1,2,3)} A B=B^{\dagger} A^{(1,3)} A B=B^{\dagger} A^{(1,2)} A B=B^{\dagger} A^{(1)} A B \\
&=B^{\dagger} A^{\dagger} A B+B^{\dagger} F_{A} W A B, \\
& B^{(1)} A^{\dagger} A B= B^{(1)} A^{(1,3,4)} A B=B^{(1)} A^{(1,2,4)} A B=B^{(1)} A^{(1,4)} A B \\
&= B^{\dagger} A^{\dagger} A B+F_{B} V_{1} A^{\dagger} A B+V_{2} E_{B} A^{\dagger} A B, \\
&= B^{(1,2)} A^{(1,3,4)} A B=B^{(1,2)} A^{(1,2,4)} A B=B^{(1,2)} A^{(1,4)} A B \\
&\left.B_{B} V_{1}\right) B\left(B^{\dagger} A^{\dagger} A B+V_{2} E_{B} A^{\dagger} A B\right),
\end{aligned}
$$




$$
\begin{aligned}
B^{(1,3,4)} A^{(1,2,3)} A B & =B^{(1,3,4)} A^{(1,3)} A B=B^{(1,3,4)} A^{(1,2)} A B=B^{(1,3,4)} A^{(1)} A B \\
& =\left(B^{\dagger}+F_{B} V E_{B}\right)\left(A^{\dagger} A B+F_{A} W A B\right), \\
B^{(1,2,4)} A^{(1,2,3)} A B & =B^{(1,2,4)} A^{(1,3)} A B=B^{(1,2,4)} A^{(1,2)} A B=B^{(1,2,4)} A^{(1)} A B \\
& =\left(B^{\dagger}+B^{\dagger} B V E_{B}\right)\left(A^{\dagger} A B+F_{A} W A B\right), \\
B^{(1,2,3)} A^{(1,2,3)} A B & =B^{(1,2,3)} A^{(1,3)} A B=B^{(1,2,3)} A^{(1,2)} A B=B^{(1,2,3)} A^{(1)} A B \\
& =\left(B^{\dagger}+F_{B} V B B^{\dagger}\right)\left(A^{\dagger} A B+F_{A} W A B\right), \\
B^{(1,4)} A^{(1,2,3)} A B & =B^{(1,4)} A^{(1,3)} A B=B^{(1,4)} A^{(1,2)} A B=B^{(1,4)} A^{(1)} A B \\
& =\left(B^{\dagger}+V E_{B}\right)\left(A^{\dagger} A B+F_{A} W A B\right), \\
B^{(1,3)} A^{(1,2,3)} A B & =B^{(1,3)} A^{(1,3)} A B=B^{(1,3)} A^{(1,2)} A B=B^{(1,3)} A^{(1)} A B \\
& =\left(B^{\dagger}+F_{B} V\right)\left(A^{\dagger} A B+F_{A} W A B\right), \\
B^{(1,2)} A^{(1,2,3)} A B & =B^{(1,2)} A^{(1,3)} A B=B^{(1,2)} A^{(1,2)} A B=B^{(1,2)} A^{(1)} A B \\
& =\left(B^{\dagger}+F_{B} V_{1}\right) B\left(B^{\dagger}+V_{2} E_{B}\right)\left(A^{\dagger} A B+F_{A} W A B\right), \\
B^{(1)} A^{(1,2,3)} A B= & B^{(1)} A^{(1,3)} A B=B^{(1)} A^{(1,2)} A B=B^{(1)} A^{(1)} A B \\
& \left(F_{B} V_{1}+V_{2} E_{B}\right)\left(A^{\dagger} A B+F_{A} W A B\right),
\end{aligned}
$$

where $V, V_{1}, V_{2}, W, W_{1}, W_{2}$ are variable matrices of appropriate sizes.

(IV) The products $A B B^{(i, \ldots, j)} A^{(i, \ldots, j)} A B$ can be written as the following four groups of expression:

$$
\begin{array}{rl}
A B & B^{\dagger} A^{(1,3,4)} A B \\
& =A B B^{\dagger} A^{(1,2,4)} A=A B B^{\dagger} A^{(1,4)} A B \\
& =A B B^{(1,3,4)} A^{\dagger} A B=A B B^{(1,3,4)} A^{(1,3,4)} A B=A B B^{(1,3,4)} A^{(1,2,4)} A B \\
& =A B B^{(1,3,4)} A^{(1,4)} A B=A B B^{(1,2,3)} A^{\dagger} A B=A B B^{(1,2,3)} A^{(1,3,4)} A B \\
& =A B B^{(1,2,3)} A^{(1,2,4)} A B=A B B^{(1,2,3)} A^{(1,4)} A B=A B B^{(1,3)} A^{\dagger} A B \\
& =A B B^{(1,3)} A^{(1,3)} A B=A B B^{(1,3)} A^{(1,2,4)} A B=A B B^{(1,3)} A^{(1,4)} A B \\
& =A B B^{\dagger} A^{\dagger} A B, \\
A B & B B^{\dagger} A^{(1,2,3)} A B \\
& =A B B^{\dagger} A^{(1,3)} A B=A B B^{\dagger} A^{(1,2)} A B \\
& =A B B^{\dagger} A^{(1)} A B=A B B^{(1,3,4)} A^{(1,2,3)} A B=A B B^{(1,3,4)} A^{(1,3)} A B \\
& =A B B^{(1,3,4)} A^{(1,2)} A B=A B B^{(1,3,4)} A^{(1)} A B=A B B^{(1,2,3)} A^{(1,2,3)} A B \\
& =A B B^{(1,2,3)} A^{(1,3)} A B=A B B^{(1,2,3)} A^{(1,2)} A B=A B B^{(1,2,3)} A^{(1)} A B \\
& =A B B^{(1,3)} A^{(1,2,3)} A B=A B B^{(1,3)} A^{(1,3)} A B=A B B^{(1,3)} A^{(1,2)} A B \\
& =A B B^{(1,3)} A^{(1)} A B=A B B^{\dagger} A^{\dagger} A B+A B B^{\dagger} F_{A} W A B,
\end{array}
$$




$$
\begin{aligned}
A B & B^{(1,2,4)} A^{\dagger} A B \\
& =A B B^{(1,2,4)} A^{(1,3,4)} A B=A B B^{(1,2,4)} A^{(1,2,4)} A B \\
& =A B B^{(1,2,4)} A^{(1,4)} A B=A B B^{(1,4)} A^{\dagger} A B=A B B^{(1,4)} A^{(1,3,4)} A B \\
& =A B B^{(1,4)} A^{(1,2,4)} A B=A B B^{(1,4)} A^{(1,4)} A B=A B B^{(1,2)} A^{\dagger} A B \\
& =A B B^{(1,2)} A^{(1,3,4)} A B=A B B^{(1,2)} A^{(1,2,4)} A B=A B B^{(1,2)} A^{(1,4)} A B \\
& =A B B^{(1)} A^{\dagger} A B=A B B^{(1)} A^{(1,3,4)} A B=A B B^{(1)} A^{(1,2,4)} A B \\
& =A B B^{(1)} A^{(1,4)} A B=A B B^{\dagger} A^{\dagger} A B+A B V E_{B} A^{\dagger} A B, \\
A B & B^{(1,2,4)} A^{(1,2,3)} A B \\
& =A B B^{(1,2,4)} A^{(1,3)} A B=A B B^{(1,2,4)} A^{(1,2)} A B \\
& =A B B^{(1,2,4)} A^{(1)} A B=A B B^{(1,4)} A^{(1,2,3)} A B=A B B^{(1,4)} A^{(1,3)} A B \\
& =A B B^{(1,4)} A^{(1,2)} A B=A B B^{(1,4)} A^{(1)} A B=A B B^{(1,2)} A^{(1,2,3)} A B \\
& =A B B^{(1,2)} A^{(1,3)} A B=A B B^{(1,2)} A^{(1,2)} A B=A B B^{(1)} A^{(1)} A B \\
& =A B B^{(1)} A^{(1,2,3)} A B=A B B^{(1)} A^{(1,3)} A B=A B B^{(1)} A^{(1,2)} A B \\
& =A B B^{(1)} A^{(1)} A B=\left(A B B^{\dagger}+A B V E_{B}\right)\left(A^{\dagger} A B+F_{A} W A B\right),
\end{aligned}
$$

where $V$ and $W$ are variable matrices of appropriate sizes.

Equations (3.2)-(3.64) show that the matrix products in (3.1) are in fact a group of linear or nonlinear matrix-valued functions with one or more independent variable matrices. Because the products $B^{(i, \ldots, j)} A^{(i, \ldots, j)}$ and their ranks may vary with respect to the choices of the variable matrices in them, the investigation of $B^{(i, \ldots, j)} A^{(i, \ldots, j)}$ is more like a pure algebraic work of characterizing performances of linear or nonlinear matrix-valued functions. As an initial step of this algebraic work, we intend to establish analytical formulas for calculating the global maximum and minimum ranks of (3.2)-(3.64) when $V, V_{1}, V_{2}, W, W_{1}$, and $W_{2}$ run over the corresponding matrix spaces. Concerning the upper and lower bounds of the ranks of (3.1), we have the following results.

Lemma 10 Let $A \in \mathbb{C}^{m \times n}$ and $B \in \mathbb{C}^{n \times p}$. Then the following inequalities:

$$
\begin{aligned}
& r\left(B^{(i, \ldots, j)} A^{(i, \ldots, j)}\right) \leq \min \left\{r\left(A^{(i, \ldots, j)}\right), r\left(B^{(i, \ldots, j)}\right)\right\} \leq \min \{m, n, p\}, \\
& r\left(B^{(i, \ldots, j)} A^{(i, \ldots, j)}\right) \geq \max \left\{0, r\left(A^{(i, \ldots, j)}\right)+r\left(B^{(i, \ldots, j)}\right)-n\right\} \geq \max \{0, r(A)+r(B)-n\}
\end{aligned}
$$

hold, where $A^{(i, \ldots, j)}$ and $B^{(i, \ldots, j)}$ are the eight commonly used generalized inverses of $A$ and $B$, respectively.

Proof The proof follows from (2.34), (2.35), and Lemma 9.

The upper and lower bounds of the ranks of (3.109) and (3.110), as shown below, are attainable for certain choices of $A^{(i, \ldots, j)}$ and $B^{(i, \ldots, j)}$. Concerning the global maximum and minimum ranks of (3.1), we have the following main results. 
Theorem 1 Let $A \in \mathbb{C}^{m \times n}$ and $B \in \mathbb{C}^{n \times p}$ be given. Then the following 126 formulas for calculating the global maximum and minimum ranks of (3.2)-(3.64) hold:

$$
\begin{aligned}
& \max _{A^{(1,3,4)}} r\left(B^{\dagger} A^{(1,3,4)}\right)=\min \{r(B), m+r(A B)-r(A)\}, \\
& \min _{A^{(1,3,4)}} r\left(B^{\dagger} A^{(1,3,4)}\right)=r(A B), \\
& \max _{A^{(1,2,4)}} r\left(B^{\dagger} A^{(1,2,4)}\right)=r(A B), \\
& \min _{A^{(1,2,4)}} r\left(B^{\dagger} A^{(1,2,4)}\right)=r(A B), \\
& \max _{A^{(1,2,3)}} r\left(B^{\dagger} A^{(1,2,3)}\right)=\min \{r(A), r(B)\}, \\
& \min _{A^{(1,2,3)}} r\left(B^{\dagger} A^{(1,2,3)}\right)=r(A)+r(B)-r\left[A^{*}, B\right], \\
& \max _{A^{(1,4)}} r\left(B^{\dagger} A^{(1,4)}\right)=\min \{r(B), m+r(A B)-r(A)\}, \\
& \min _{A^{(1,4)}} r\left(B^{\dagger} A^{(1,4)}\right)=r(A B), \\
& \max _{A^{(1,3)}} r\left(B^{\dagger} A^{(1,3)}\right)=\min \{m, r(B)\}, \\
& \min _{A^{(1,3)}} r\left(B^{\dagger} A^{(1,3)}\right)=r(A)+r(B)-r\left[A^{*}, B\right], \\
& \max _{A^{(1,2)}} r\left(B^{\dagger} A^{(1,2)}\right)=\min \{r(A), r(B)\}, \\
& \min _{A^{(1,2)}} r\left(B^{\dagger} A^{(1,2)}\right)=r(A)+r(B)-r\left[A^{*}, B\right], \\
& \max _{A^{(1)}} r\left(B^{\dagger} A^{(1)}\right)=\min \{m, r(B)\}, \\
& \min _{A^{(1)}} r\left(B^{\dagger} A^{(1)}\right)=r(A)+r(B)-r\left[A^{*}, B\right], \\
& \max _{B^{(1,3,4)}} r\left(B^{(1,3,4)} A^{\dagger}\right)=\min \{r(A), p+r(A B)-r(B)\}, \\
& \min _{B^{(1,3,4)}} r\left(B^{(1,3,4)} A^{\dagger}\right)=r(A B), \\
& \max _{B^{(1,3,4)}, A^{(1,3,4)}} r\left(B^{(1,3,4)} A^{(1,3,4)}\right)=\min \{m, n, p, m+p+r(A B)-r(A)-r(B)\}, \\
& \min _{B^{(1,3,4)}, A^{(1,3,4)}} r\left(B^{(1,3,4)} A^{(1,3,4)}\right)=r(A B), \\
& \max _{B^{(1,3,4)}, A^{(1,2,4)}} r\left(B^{(1,3,4)} A^{(1,2,4)}\right)=\min \{r(A), p+r(A B)-r(B)\}, \\
& \min _{B^{(1,3,4)}, A^{(1,2,4)}} r\left(B^{(1,3,4)} A^{(1,2,4)}\right)=r(A B), \\
& \max _{B^{(1,3,4)}, A^{(1,2,3)}} r\left(B^{(1,3,4)} A^{(1,2,3)}\right)=\min \{p, r(A)\}, \\
& \min _{B^{(1,3,4)}, A^{(1,2,3)}} r\left(B^{(1,3,4)} A^{(1,2,3)}\right)=r(A)+r(B)-r\left[A^{*}, B\right], \\
& \max _{B^{(1,3,4)}, A^{(1,4)}} r\left(B^{(1,3,4)} A^{(1,4)}\right)=\min \{m, n, p, m+p+r(A B)-r(A)-r(B)\},
\end{aligned}
$$




$$
\begin{aligned}
& \min _{B^{(1,3,4)}, A^{(1,4)}} r\left(B^{(1,3,4)} A^{(1,4)}\right)=r(A B), \\
& \max _{B^{(1,3,4)}, A^{(1,3)}} r\left(B^{(1,3,4)} A^{(1,3)}\right)=\min \{m, n, p\}, \\
& \min _{B^{(1,3,4)}, B^{(1,3)}} r\left(B^{(1,3,4)} A^{(1,3)}\right)=r(A)+r(B)-r\left[A^{*}, B\right], \\
& \max _{B^{(1,3,4)}, A^{(1,2)}} r\left(B^{(1,3,4)} A^{(1,2)}\right)=\min \{p, r(A)\}, \\
& \min _{B^{(1,3,4)}, A^{(1,2)}} r\left(B^{(1,3,4)} A^{(1,2)}\right)=r(A)+r(B)-r\left[A^{*}, B\right], \\
& \max _{B^{(1,3,4)}, A^{(1)}} r\left(B^{(1,3,4)} A^{(1)}\right)=\min \{m, n, p\}, \\
& \min _{B^{(1,3,4)}, A^{(1)}} r\left(B^{(1,3,4)} A^{(1)}\right)=r(A)+r(B)-r\left[A^{*}, B\right], \\
& \max _{B^{(1,2,4)}} r\left(B^{(1,2,4)} A^{\dagger}\right)=\min \{r(A), r(B)\}, \\
& \min _{B^{(1,2,4)}} r\left(B^{(1,2,4)} A^{\dagger}\right)=r(A)+r(B)-r\left[A^{*}, B\right], \\
& \max _{B^{(1,2,4)}, A^{(1,3,4)}} r\left(B^{(1,2,4)} A^{(1,3,4)}\right)=\min \{m, r(B)\}, \\
& \min _{B^{(1,2,4)}, A^{(1,3,4)}} r\left(B^{(1,2,4)} A^{(1,3,4)}\right)=r(A)+r(B)-r\left[A^{*}, B\right], \\
& \max _{B^{(1,2,4)}, A^{(1,2,4)}} r\left(B^{(1,2,4)} A^{(1,2,4)}\right)=\min \{r(A), r(B)\}, \\
& \min _{B^{(1,2,4)}, A^{(1,2,4)}} r\left(B^{(1,2,4)} A^{(1,2,4)}\right)=r(A)+r(B)-r\left[A^{*}, B\right], \\
& \max _{B^{(1,2,4)}, A^{(1,2,3)}} r\left(B^{(1,2,4)} A^{(1,2,3)}\right)=\min \{r(A), r(B)\}, \\
& \min _{B^{(1,2,4)}, A^{(1,2,3)}} r\left(B^{(1,2,4)} A^{(1,2,3)}\right)=\max \{0, r(A)+r(B)-n\}, \\
& \max _{B^{(1,2,4)}, A^{(1,4)}} r\left(B^{(1,2,4)} A^{(1,4)}\right)=\min \{m, r(B)\}, \\
& \min _{B^{(1,2,4)}, A^{(1,4)}} r\left(B^{(1,2,4)} A^{(1,4)}\right)=r(A)+r(B)-r\left[A^{*}, B\right], \\
& \max _{B^{(1,2,4)}, A^{(1,3)}} r\left(B^{(1,2,4)} A^{(1,3)}\right)=\min \{m, r(B)\}, \\
& \min _{B^{(1,2,4)}, A^{(1,3)}} r\left(B^{(1,2,4)} A^{(1,3)}\right)=\max \{0, r(A)+r(B)-n\}, \\
& \max _{B^{(1,2,4)}, A^{(1,2)}} r\left(B^{(1,2,4)} A^{(1,2)}\right)=\min \{r(A), r(B)\} \\
& \min _{B^{(1,2,4)}, A^{(1,2)}} r\left(B^{(1,2,4)} A^{(1,2)}\right)=\max \{0, r(A)+r(B)-n\}, \\
& \max _{B^{(1,2,4)}, A^{(1)}} r\left(B^{(1,2,4)} A^{(1)}\right)=\min \{m, r(B)\}, \\
& \min _{B^{(1,2,4)}, A^{(1)}} r\left(B^{(1,2,4)} A^{(1)}\right)=\max \{0, r(A)+r(B)-n\}, \\
& \max _{B^{(1,2,3)}} r\left(B^{(1,2,3)} A^{\dagger}\right)=r(A B),
\end{aligned}
$$




$$
\begin{aligned}
& \min _{B^{(1,2,3)}} r\left(B^{(1,2,3)} A^{\dagger}\right)=r(A B), \\
& \max _{B^{(1,2,3)}, A^{(1,3,4)}} r\left(B^{(1,2,3)} A^{(1,3,4)}\right)=\min \{r(B), m+r(A B)-r(A)\}, \\
& \min _{B^{(1,2,3)}, A^{(1,3,4)}} r\left(B^{(1,2,3)} A^{(1,3,4)}\right)=r(A B), \\
& \max _{B^{(1,2,3)}, A^{(1,2,4)}} r\left(B^{(1,2,3)} A^{(1,2,4)}\right)=r(A B), \\
& \min _{B^{(1,2,3)}, A^{(1,2,4)}} r\left(B^{(1,2,3)} A^{(1,2,4)}\right)=r(A B), \\
& \max _{B^{(1,2,3)}, A^{(1,2,3)}} r\left(B^{(1,2,3)} A^{(1,2,3)}\right)=\min \{r(A), r(B)\}, \\
& \min _{B^{(1,2,3)}, A^{(1,2,3)}} r\left(B^{(1,2,3)} A^{(1,2,3)}\right)=r(A)+r(B)-r\left[A^{*}, B\right], \\
& \max _{B^{(1,2,3)}, A^{(1,4)}} r\left(B^{(1,2,3)} A^{(1,4)}\right)=\min \{r(B), m+r(A B)-r(A)\}, \\
& \min _{B^{(1,2,3)}, A^{(1,4)}} r\left(B^{(1,2,3)} A^{(1,4)}\right)=r(A B), \\
& \max _{B^{(1,2,3)}, A^{(1,3)}} r\left(B^{(1,2,3)} A^{(1,3)}\right)=\min \{m, r(B)\}, \\
& \min _{B^{(1,2,3)}, A^{(1,3)}} r\left(B^{(1,2,3)} A^{(1,3)}\right)=r(A)+r(B)-r\left[A^{*}, B\right], \\
& \max _{B^{(1,2,3)}, A^{(1,2)}} r\left(B^{(1,2,3)} A^{(1,2)}\right)=\min \{r(A), r(B)\}, \\
& \min _{B^{(1,2,3)}, A^{(1,2)}} r\left(B^{(1,2,3)} A^{(1,2)}\right)=r(A)+r(B)-r\left[A^{*}, B\right], \\
& \max _{B^{(1,2,3)}, A^{(1)}} r\left(B^{(1,2,3)} A^{(1)}\right)=\min \{m, r(B)\}, \\
& \min _{B^{(1,2,3)}, A^{(1)}} r\left(B^{(1,2,3)} A^{(1)}\right)=r(A)+r(B)-r\left[A^{*}, B\right], \\
& \max _{B^{(1,4)}} r\left(B^{(1,4)} A^{\dagger}\right)=\min \{p, r(A)\}, \\
& \min _{B^{(1,4)}} r\left(B^{(1,4)} A^{\dagger}\right)=r(A)+r(B)-r\left[A^{*}, B\right], \\
& \max _{B^{(1,4)}, A^{(1,3,4)}} r\left(B^{(1,4)} A^{(1,3,4)}\right)=\min \{m, n, p\}, \\
& \min _{B^{(1,4)}, A^{(1,3,4)}} r\left(B^{(1,4)} A^{(1,3,4)}\right)=r(A)+r(B)-r\left[A^{*}, B\right], \\
& \max _{B^{(1,4)}, A^{(1,2,4)}} r\left(B^{(1,4)} A^{(1,2,4)}\right)=\min \{p, r(A)\}, \\
& \min _{B^{(1,4)}, A^{(1,2,4)}} r\left(B^{(1,4)} A^{(1,2,4)}\right)=r(A)+r(B)-r\left[A^{*}, B\right], \\
& \max _{B^{(1,4)}, A^{(1,2,3)}} r\left(B^{(1,4)} A^{(1,2,3)}\right)=\min \{p, r(A)\}, \\
& \min _{B^{(1,4)}, A^{(1,2,3)}} r\left(B^{(1,4)} A^{(1,2,3)}\right)=\max \{0, r(A)+r(B)-n\}, \\
& \max _{B^{(1,4)}, A^{(1,4)}} r\left(B^{(1,4)} A^{(1,4)}\right)=\min \{m, n, p\},
\end{aligned}
$$




$$
\begin{aligned}
& \min _{B^{(1,4)}, A^{(1,4)}} r\left(B^{(1,4)} A^{(1,4)}\right)=r(A)+r(B)-r\left[A^{*}, B\right], \\
& \max _{B^{(1,4)}, A^{(1,3)}} r\left(B^{(1,4)} A^{(1,3)}\right)=\min \{m, n, p\}, \\
& \min _{B^{(1,4)}, A^{(1,3)}} r\left(B^{(1,4)} A^{(1,3)}\right)=\max \{0, r(A)+r(B)-n\}, \\
& \max _{B^{(1,4)}, A^{(1,2)}} r\left(B^{(1,4)} A^{(1,2)}\right)=\min \{p, r(A)\}, \\
& \min _{B^{(1,4)}, A^{(1,2)}} r\left(B^{(1,4)} A^{(1,2)}\right)=\max \{0, r(A)+r(B)-n\}, \\
& \max _{B^{(1,4)}, A^{(1)}} r\left(B^{(1,4)} A^{(1)}\right)=\min \{m, n, p\}, \\
& \min _{B^{(1,4)}, A^{(1)}} r\left(B^{(1,4)} A^{(1)}\right)=\max \{0, r(A)+r(B)-n\}, \\
& \max _{B^{(1,3)}} r\left(B^{(1,3)} A^{\dagger}\right)=\min \{r(A), p+r(A B)-r(B)\}, \\
& \min _{B^{(1,3)}} r\left(B^{(1,3)} A^{\dagger}\right)=r(A B), \\
& \max _{B^{(1,3)}, A^{(1,3,4)}} r\left(B^{(1,3)} A^{(1,3,4)}\right)=\min \{m, n, p, m+p+r(A B)-r(A)-r(B)\}, \\
& \min _{B^{(1,3)}, A^{(1,3,4)}} r\left(B^{(1,3)} A^{(1,3,4)}\right)=r(A B), \\
& \max _{B^{(1,3)}, A^{(1,2,4)}} r\left(B^{(1,3)} A^{(1,2,4)}\right)=\min \{r(A), p+r(A B)-r(B)\}, \\
& \min _{B^{(1,3)}, A^{(1,2,4)}} r\left(B^{(1,3)} A^{(1,2,4)}\right)=r(A B), \\
& \max _{B^{(1,3)}, A^{(1,2,3)}} r\left(B^{(1,3)} A^{(1,2,3)}\right)=\min \{p, r(A)\}, \\
& \min _{B^{(1,3)}, A^{(1,2,3)}} r\left(B^{(1,3)} A^{(1,2,3)}\right)=r(A)+r(B)-r\left[A^{*}, B\right], \\
& \max _{B^{(1,3)}, A^{(1,4)}} r\left(B^{(1,3)} A^{(1,4)}\right)=\min \{m, n, p, m+p+r(A B)-r(A)-r(B)\}, \\
& \min _{B^{(1,3)}, A^{(1,4)}} r\left(B^{(1,3)} A^{(1,4)}\right)=r(A B), \\
& \max _{B^{(1,3)}, A^{(1,3)}} r\left(B^{(1,3)} A^{(1,3)}\right)=\min \{m, n, p\}, \\
& \min _{B^{(1,3)}, A^{(1,3)}} r\left(B^{(1,3)} A^{(1,3)}\right)=r(A)+r(B)-r\left[A^{*}, B\right], \\
& \max _{B^{(1,3)}, A^{(1,2)}} r\left(B^{(1,3)} A^{(1,2)}\right)=\min \{p, r(A)\}, \\
& \min _{B^{(1,3)}, A^{(1,2)}} r\left(B^{(1,3)} A^{(1,2)}\right)=r(A)+r(B)-r\left[A^{*}, B\right], \\
& \max _{B^{(1,3)}, A^{(1)}} r\left(B^{(1,3)} A^{(1)}\right)=\min \{m, n, p\}, \\
& \min _{B^{(1,3)}, A^{(1)}} r\left(B^{(1,3)} A^{(1)}\right)=r(A)+r(B)-r\left[A^{*}, B\right], \\
& \max _{B^{(1,2)}} r\left(B^{(1,2)} A^{\dagger}\right)=\min \{r(A), r(B)\},
\end{aligned}
$$




$$
\begin{aligned}
& \min _{B^{(1,2)}} r\left(B^{(1,2)} A^{\dagger}\right)=r(A)+r(B)-r\left[A^{*}, B\right], \\
& \max _{B^{(1,2)}, A^{(1,3,4)}} r\left(B^{(1,2)} A^{(1,3,4)}\right)=\min \{m, r(B)\}, \\
& \min _{B^{(1,2)}, A^{(1,3,4)}} r\left(B^{(1,2)} A^{(1,3,4)}\right)=r(A)+r(B)-r\left[A^{*}, B\right], \\
& \max _{B^{(1,2)}, A^{(1,2,4)}} r\left(B^{(1,2)} A^{(1,2,4)}\right)=\min \{r(A), r(B)\}, \\
& \min _{B^{(1,2)}, A^{(1,2,4)}} r\left(B^{(1,2)} A^{(1,2,4)}\right)=r(A)+r(B)-r\left[A^{*}, B\right], \\
& \max _{B^{(1,2)}, A^{(1,2,3)}} r\left(B^{(1,2)} A^{(1,2,3)}\right)=\min \{r(A), r(B)\}, \\
& \min _{B^{(1,2)}, A^{(1,2,3)}} r\left(B^{(1,2)} A^{(1,2,3)}\right)=\max \{0, r(A)+r(B)-n\}, \\
& \max _{B^{(1,2)}, A^{(1,4)}} r\left(B^{(1,2)} A^{(1,4)}\right)=\min \{m, r(B)\}, \\
& \min _{B^{(1,2)}, A^{(1,4)}} r\left(B^{(1,2)} A^{(1,4)}\right)=r(A)+r(B)-r\left[A^{*}, B\right], \\
& \max _{B^{(1,2)}, A^{(1,3)}} r\left(B^{(1,2)} A^{(1,3)}\right)=\min \{m, r(B)\}, \\
& \min _{B^{(1,2)}, A^{(1,3)}} r\left(B^{(1,2)} A^{(1,3)}\right)=\max \{0, r(A)+r(B)-n\}, \\
& \max _{B^{(1,2)}, A^{(1,2)}} r\left(B^{(1,2)} A^{(1,2)}\right)=\min \{r(A), r(B)\}, \\
& \min _{B^{(1,2)}, A^{(1,2)}} r\left(B^{(1,2)} A^{(1,2)}\right)=\max \{0, r(A)+r(B)-n\}, \\
& \max _{B^{(1,2)}, A^{(1)}} r\left(B^{(1,2)} A^{(1)}\right)=\min \{m, r(B)\}, \\
& \min _{B^{(1,2)}, A^{(1)}} r\left(B^{(1,2)} A^{(1)}\right)=\max \{0, r(A)+r(B)-n\}, \\
& \max _{B^{(1)}} r\left(B^{(1)} A^{\dagger}\right)=\min \{p, r(A)\}, \\
& \min _{B^{(1)}} r\left(B^{(1)} A^{\dagger}\right)=r(A)+r(B)-r\left[A^{*}, B\right], \\
& \max _{B^{(1)}, A^{(1,3,4)}} r\left(B^{(1)} A^{(1,3,4)}\right)=\min \{m, n, p\}, \\
& \min _{B^{(1)}, A^{(1,3,4)}} r\left(B^{(1)} A^{(1,3,4)}\right)=r(A)+r(B)-r\left[A^{*}, B\right], \\
& \max _{B^{(1)}, A^{(1,2,4)}} r\left(B^{(1)} A^{(1,2,4)}\right)=\min \{p, r(A)\}, \\
& \min _{B^{(1)}, A^{(1,2,4)}} r\left(B^{(1)} A^{(1,2,4)}\right)=r(A)+r(B)-r\left[A^{*}, B\right], \\
& \max _{B^{(1)}, A^{(1,2,3)}} r\left(B^{(1)} A^{(1,2,3)}\right)=\min \{p, r(A)\}, \\
& \min _{B^{(1)}, A^{(1,2,3)}} r\left(B^{(1)} A^{(1,2,3)}\right)=\max \{0, r(A)+r(B)-n\}, \\
& \max _{B^{(1)}, A^{(1,4)}} r\left(B^{(1)} A^{(1,4)}\right)=\min \{m, n, p\},
\end{aligned}
$$




$$
\begin{aligned}
& \min _{B^{(1)}, A^{(1,4)}} r\left(B^{(1)} A^{(1,4)}\right)=r(A)+r(B)-r\left[A^{*}, B\right], \\
& \max _{B^{(1)}, A^{(1,3)}} r\left(B^{(1)} A^{(1,3)}\right)=\min \{m, n, p\}, \\
& \min _{B^{(1)}, A^{(1,3)}} r\left(B^{(1)} A^{(1,3)}\right)=\max \{0, r(A)+r(B)-n\}, \\
& \max _{B^{(1)}, A^{(1,2)}} r\left(B^{(1)} A^{(1,2)}\right)=\min \{p, r(A)\}, \\
& \min _{B^{(1)}, A^{(1,2)}} r\left(B^{(1)} A^{(1,2)}\right)=\max \{0, r(A)+r(B)-n\}, \\
& \max _{B^{(1)}, A^{(1)}} r\left(B^{(1)} A^{(1)}\right)=\min \{m, n, p\}, \\
& \min _{B^{(1)}, A^{(1)}} r\left(B^{(1)} A^{(1)}\right)=\max \{0, r(A)+r(B)-n\} .
\end{aligned}
$$

Proof Note from (2.36) that $r\left(B^{\dagger} A^{(i, \ldots, j)}\right)=r\left(B^{*} A^{(i, \ldots, j)}\right)$ holds for all $A^{(i, \ldots, j)}$. Applying (2.52)(2.65) to it yields

$$
\begin{aligned}
& \max _{A^{(1,3,4)}} r\left(B^{*} A^{(1,3,4)}\right)=\min \left\{r\left(B^{*}\right), m+r\left(B^{*} A^{*}\right)-r(A)\right\} \\
& =\min \{r(B), m+r(A B)-r(A)\}, \\
& \min _{A^{(1,3,4)}} r\left(B^{*} A^{(1,3,4)}\right)=r\left(B^{*} A^{*}\right)=r(A B), \\
& \max _{A^{(1,2,4)}} r\left(B^{*} A^{(1,2,4)}\right)=r\left(B^{*} A^{*}\right)=r(A B), \\
& \min _{A^{(1,2,4)}} r\left(B^{*} A^{(1,2,4)}\right)=r\left(B^{*} A^{*}\right)=r(A B), \\
& \max _{A^{(1,2,3)}} r\left(B^{*} A^{(1,2,3)}\right)=\min \left\{r(A), r\left(B^{*}\right)\right\}=\min \{r(A), r(B)\}, \\
& \min _{A^{(1,2,3)}} r\left(B^{*} A^{(1,2,3)}\right)=r(A)+r\left(B^{*}\right)-r\left[A^{*},\left(B^{*}\right)^{*}\right]=r(A)+r(B)-r\left[A^{*}, B\right], \\
& \max _{A^{(1,4)}} r\left(B^{*} A^{(1,4)}\right)=\min \left\{r\left(B^{*}\right), m+r\left(B^{*} A^{*}\right)-r(A)\right\}=\min \{r(B), m+r(A B)-r(A)\}, \\
& \min _{A^{(1,4)}} r\left(B^{*} A^{(1,4)}\right)=r\left(B^{*} A^{*}\right)=r(A B), \\
& \max _{A^{(1,3)}} r\left(B^{*} A^{(1,3)}\right)=\min \left\{m, r\left(B^{*}\right)\right\}=\min \{m, r(B)\}, \\
& \min _{A^{(1,3)}} r\left(B^{*} A^{(1,3)}\right)=r(A)+r\left(B^{*}\right)-r\left[A^{*},\left(B^{*}\right)^{*}\right]=r(A)+r(B)-r\left[A^{*}, B\right], \\
& \max _{A^{(1,2)}} r\left(B^{*} A^{(1,2)}\right)=\min \left\{r(A), r\left(B^{*}\right)\right\}=\min \{r(A), r(B)\}, \\
& \min _{A^{(1,2)}} r\left(B^{*} A^{(1,2)}\right)=r(A)+r\left(B^{*}\right)-r\left[A^{*},\left(B^{*}\right)^{*}\right]=r(A)+r(B)-r\left[A^{*}, B\right], \\
& \max _{A^{(1)}} r\left(B^{*} A^{(1)}\right)=\min \left\{m, r\left(B^{*}\right)\right\}=\min \{m, r(B)\}, \\
& \min _{A^{(1)}} r\left(B^{*} A^{(1)}\right)=r(A)+r\left(B^{*}\right)-r\left[A^{*},\left(B^{*}\right)^{*}\right]=r(A)+r(B)-r\left[A^{*}, B\right], \\
&
\end{aligned}
$$

thus establishing (3.111)-(3.124). 
Note from (2.36) that $r\left(B^{(i, \ldots, j)} A^{\dagger}\right)=r\left(B^{(i, \ldots, j)} A^{*}\right)$ holds for all $B^{(i, \ldots, j)}$. Hence, (3.125), (3.126), (3.141), (3.142), (3.157), (3.158), (3.173), (3.174), (3.189), (3.190), (3.205), (3.206), (3.221), and (3.222) follow from (2.38)-(2.51).

Applying (2.66) and (2.67) to (3.14), we obtain

$$
\begin{aligned}
& \max _{B^{(1,3,4)}, A^{(1,3)}} r\left(B^{(1,3,4)} A^{(1,3)}\right) \\
& =\max _{V, W} r\left[\left(B^{\dagger}+F_{B} V E_{B}\right)\left(A^{\dagger}+F_{A} W\right)\right] \\
& =\min \left\{r\left[B^{\dagger} A^{\dagger}, B^{\dagger} F_{A}, F_{B}\right], r\left[\begin{array}{c}
B^{\dagger} A^{\dagger} \\
E_{B} A^{\dagger} \\
I_{m}
\end{array}\right], r\left[\begin{array}{cc}
B^{\dagger} A^{\dagger} & F_{B} \\
I_{m} & 0
\end{array}\right], r\left[\begin{array}{cc}
B^{\dagger} A^{\dagger} & B^{\dagger} F_{A} \\
E_{B} A^{\dagger} & E_{B} F_{A}
\end{array}\right]\right\}, \\
& \min _{B^{(1,3,4)}, A^{(1,3)}} r\left(B^{(1,3,4)} A^{(1,3)}\right)=\min _{V, W} r\left[\left(B^{\dagger}+F_{B} V E_{B}\right)\left(A^{\dagger}+F_{A} W\right)\right] \\
& =r\left[\begin{array}{c}
B^{\dagger} A^{\dagger} \\
E_{B} A^{\dagger} \\
I_{m}
\end{array}\right]+r\left[B^{\dagger} A^{\dagger}, B^{\dagger} F_{A}, F_{B}\right]+\max \left\{s_{1}, s_{2}\right\},
\end{aligned}
$$

where

$$
\begin{aligned}
& s_{1}=r\left[\begin{array}{cc}
B^{\dagger} A^{\dagger} & F_{B} \\
I_{m} & 0
\end{array}\right]-r\left[\begin{array}{ccc}
B^{\dagger} A^{\dagger} & F_{B} & B^{\dagger} F_{A} \\
I_{m} & 0 & 0
\end{array}\right]-r\left[\begin{array}{cc}
B^{\dagger} A^{\dagger} & F_{B} \\
I_{m} & 0 \\
E_{B} A^{\dagger} & 0
\end{array}\right], \\
& s_{2}=r\left[\begin{array}{cc}
B^{\dagger} A^{\dagger} & B^{\dagger} F_{A} \\
E_{B} A^{\dagger} & E_{B} F_{A}
\end{array}\right]-r\left[\begin{array}{ccc}
B^{\dagger} A^{\dagger} & B^{\dagger} F_{A} & F_{B} \\
E_{B} A^{\dagger} & E_{B} F_{A} & 0
\end{array}\right]-r\left[\begin{array}{cc}
B^{\dagger} A^{\dagger} & B^{\dagger} F_{A} \\
E_{B} A^{\dagger} & E_{B} F_{A} \\
I_{m} & 0
\end{array}\right] .
\end{aligned}
$$

Simplifying the formulas by (2.29)-(2.31) gives

$$
\begin{gathered}
r\left[B^{\dagger} A^{\dagger}, B^{\dagger} F_{A}, F_{B}\right]=r\left[B^{\dagger} A^{\dagger}, B^{\dagger}, F_{B}\right]=r\left[B^{\dagger} A^{\dagger}, B^{\dagger}, I_{p}\right]=p, \\
r\left[\begin{array}{c}
B^{\dagger} A^{\dagger} \\
E_{B} A^{\dagger} \\
I_{m}
\end{array}\right]=m, \quad r\left[\begin{array}{cc}
B^{\dagger} A^{\dagger} & F_{B} \\
I_{m} & 0
\end{array}\right]=m+r\left(F_{B}\right)=m+p-r(B), \\
r\left[\begin{array}{cc}
B^{\dagger} A^{\dagger} & B^{\dagger} F_{A} \\
E_{B} A^{\dagger} & E_{B} F_{A}
\end{array}\right]=r\left[\begin{array}{ccc}
B^{\dagger} A^{\dagger} & B^{\dagger} & 0 \\
A^{\dagger} & I_{n} & B \\
0 & A & 0
\end{array}\right]-r(A)-r(B) \\
=r\left[\begin{array}{ccc}
0 & 0 & -B^{\dagger} B \\
0 & I_{n} & 0 \\
-A^{\dagger} A & 0 & 0
\end{array}\right]-r(A)-r(B)=n, \\
r\left[\begin{array}{ccc}
B^{\dagger} A^{\dagger} & F_{B} & B^{\dagger} F_{A} \\
I_{m} & 0 & 0
\end{array}\right]=m+r\left(F_{B}\right)+r\left(B^{\dagger} F_{A}\right) \\
=m+p-r(A)-r(B)+r\left[A^{*}, B\right],
\end{gathered}
$$




$$
\begin{aligned}
& r\left[\begin{array}{cc}
B^{\dagger} A^{\dagger} & F_{B} \\
I_{m} & 0 \\
E_{B} A^{\dagger} & 0
\end{array}\right]=m+r\left(F_{B}\right)=m+p-r(B), \\
& r\left[\begin{array}{ccc}
B^{\dagger} A^{\dagger} & B^{\dagger} F_{A} & F_{B} \\
E_{B} A^{\dagger} & E_{B} F_{A} & 0
\end{array}\right]=r\left[\begin{array}{cc}
B^{\dagger} A^{\dagger} & B^{\dagger} F_{A} \\
E_{B} A^{\dagger} & E_{B} F_{A}
\end{array}\right]+r\left(F_{B}\right)=n+p-r(B), \\
& r\left[\begin{array}{cc}
B^{\dagger} A^{\dagger} & B^{\dagger} F_{A} \\
E_{B} A^{\dagger} & E_{B} F_{A} \\
I_{m} & 0
\end{array}\right]=m+r\left[\begin{array}{l}
B^{\dagger} F_{A} \\
E_{B} F_{A}
\end{array}\right]=m+r\left(F_{A}\right)=m+n-r(A) .
\end{aligned}
$$

Substituting (3.239)-(3.245) into (3.237) and (3.238) and simplifying leads to (3.135) and (3.136).

Also from (2.25), (2.26), and symmetry of patterns, we obtain from (3.135) and (3.136) the following two rank equalities:

$$
\begin{aligned}
\max _{B^{(1,4)}, A^{(1,3,4)}} r\left(B^{(1,4)} A^{(1,3,4)}\right) & =\max _{B^{(1,4)}, A^{(1,3,4)}} r\left[\left(B^{(1,4)} A^{(1,3,4)}\right)^{*}\right] \\
& =\max _{\left(B^{*}\right)^{(1,3)},\left(A^{*}\right)^{(1,3,4)}} r\left[\left(A^{*}\right)^{(1,3,4)}\left(B^{*}\right)^{(1,3)}\right] \\
& =\min \{m, n, p\}, \\
\min _{B^{(1,4)}, A^{(1,3,4)}} r\left(B^{(1,4)} A^{(1,3,4)}\right) & =\min _{B^{(1,4)}, A^{(1,3,4)}} r\left[\left(B^{(1,4)} A^{(1,3,4)}\right)^{*}\right] \\
& =\min _{\left(B^{*}(1,3),\left(A^{*}\right)^{(1,3,4)}\right.} r\left[\left(A^{*}\right)^{(1,3,4)}\left(B^{*}\right)^{(1,3)}\right] \\
& =r(A)+r(B)-r\left[A^{*}, B\right],
\end{aligned}
$$

thus establishing (3.175) and (3.176).

Note from the definitions of $\{1,2\}-,\{1,2,3\}$-, and $\{1,2,4\}$-inverses of matrices that

$$
\begin{aligned}
r\left(B^{(1,2,4)} A^{(1,2,3)}\right) & =r\left(B^{(1,2)} A^{(1,2,3)}\right)=r\left(B^{(1,2,4)} A^{(1,2)}\right)=r\left(B^{(1,2)} A^{(1,2)}\right) \\
& =r\left(B B^{(1,2)} A^{(1,2)} A\right)
\end{aligned}
$$

always hold for all $A^{(1,2)}, A^{(1,2,3)}, B^{(1,2)}$, and $B^{(1,2,4)}$. Also see from (3.55) that

$$
B B^{(1,2)} A^{(1,2)} A=\left(B B^{\dagger}+B V E_{B}\right)\left(A^{\dagger} A+F_{A} W A\right) .
$$

Applying (2.66) and (2.67) to (3.247), we obtain

$$
\begin{aligned}
\max _{V, W} r & {\left[\left(B B^{\dagger}+B V E_{B}\right)\left(A^{\dagger} A+F_{A} W A\right)\right] } \\
= & \min \left\{r\left[B B^{\dagger} A^{\dagger} A, B B^{\dagger} F_{A}, B\right], r\left[\begin{array}{c}
B B^{\dagger} A^{\dagger} A \\
E_{B} A^{\dagger} A \\
A
\end{array}\right],\right. \\
& \left.r\left[\begin{array}{cc}
B B^{\dagger} A^{\dagger} A & B \\
A & 0
\end{array}\right], r\left[\begin{array}{cc}
B B^{\dagger} A^{\dagger} A & B B^{\dagger} F_{A} \\
E_{B} A^{\dagger} A & E_{B} F_{A}
\end{array}\right]\right\},
\end{aligned}
$$




$$
\begin{aligned}
\min _{V, W} r & {\left[\left(B B^{\dagger}+B V E_{B}\right)\left(A^{\dagger} A+F_{A} W A\right)\right] } \\
& =r\left[\begin{array}{c}
B B^{\dagger} A^{\dagger} A \\
E_{B} A^{\dagger} A \\
A
\end{array}\right]+r\left[B B^{\dagger} A^{\dagger} A, B B^{\dagger} F_{A}, B\right]+\max \left\{s_{1}, s_{2}\right\},
\end{aligned}
$$

where

$$
\begin{aligned}
& s_{1}=r\left[\begin{array}{cc}
B B^{\dagger} A^{\dagger} A & B \\
A & 0
\end{array}\right]-r\left[\begin{array}{ccc}
B B^{\dagger} A^{\dagger} A & B & B B^{\dagger} F_{A} \\
A & 0 & 0
\end{array}\right]-r\left[\begin{array}{cc}
B B^{\dagger} A^{\dagger} A & B \\
A & 0 \\
E_{B} A^{\dagger} A & 0
\end{array}\right], \\
& s_{2}=r\left[\begin{array}{cc}
B B^{\dagger} A^{\dagger} A & B B^{\dagger} F_{A} \\
E_{B} A^{\dagger} A & E_{B} F_{A}
\end{array}\right]-r\left[\begin{array}{ccc}
B B^{\dagger} A^{\dagger} A & B B^{\dagger} F_{A} & B \\
E_{B} A^{\dagger} A & E_{B} F_{A} & 0
\end{array}\right]-r\left[\begin{array}{cc}
B B^{\dagger} A^{\dagger} A & B B^{\dagger} F_{A} \\
E_{B} A^{\dagger} A & E_{B} F_{A} \\
A & 0
\end{array}\right] .
\end{aligned}
$$

Simplifying the formulas by (2.29)-(2.31) gives

$$
\begin{aligned}
r\left[B B^{\dagger} A^{\dagger} A, B B^{\dagger} F_{A}, B\right]=r(B), & r\left[\begin{array}{c}
B B^{\dagger} A^{\dagger} A \\
E_{B} A^{\dagger} A \\
A
\end{array}\right]=r(A), \\
r\left[\begin{array}{cr}
B B^{\dagger} A^{\dagger} A & B \\
A & 0
\end{array}\right] & =r\left[\begin{array}{ccc}
B B^{\dagger} A^{\dagger} A & B & B B^{\dagger} F_{A} \\
A & 0 & 0
\end{array}\right]=r\left[\begin{array}{cc}
B B^{\dagger} A^{\dagger} A & B \\
A & 0 \\
E_{B} A^{\dagger} A & 0
\end{array}\right] \\
& =r(A)+r(B),
\end{aligned}
$$

Substituting (3.250)-(3.254) into (3.248) and (3.249) and combining (3.246) and (3.247) leads to (3.147), (3.148), (3.153), (3.211), (3.217), and (3.218), respectively.

Note from (2.19)-(2.24) that

$$
\begin{aligned}
& \left\{B^{(1,3,4)} A^{(1,3)}\right\} \subseteq\left\{B^{(1,3,4)} A^{(1)}\right\} \subseteq\left\{B^{(1)} A^{(1)}\right\}, \\
& \left\{B^{(1,3,4)} A^{(1,3)}\right\} \subseteq\left\{B^{(1,4)} A^{(1,3)}\right\} \subseteq\left\{B^{(1)} A^{(1,3)}\right\}, \\
& \left\{B^{(1,3,4)} A^{(1,3)}\right\} \subseteq\left\{B^{(1,3)} A^{(1,3)}\right\} \subseteq\left\{B^{(1,3)} A^{(1)}\right\}, \\
& \left\{B^{(1,4)} A^{(1,3,4)}\right\} \subseteq\left\{B^{(1)} A^{(1,3,4)}\right\}, \\
& \left\{B^{(1,4)} A^{(1,3,4)}\right\} \subseteq\left\{B^{(1,4)} A^{(1,4)}\right\} \subseteq\left\{B^{(1)} A^{(1,4)}\right\}, \\
& \left\{B^{(1,4)} A^{(1,3,4)}\right\} \subseteq\left\{B^{(1,4)} A^{(1)}\right\},
\end{aligned}
$$


and

$$
\begin{aligned}
& \left\{B^{(1,2,4)} A^{(1,2,3)}\right\} \subseteq\left\{B^{(1,2,4)} A^{(1,3)}\right\} \subseteq\left\{B^{(1,2,4)} A^{(1)}\right\} \subseteq\left\{B^{(1,4)} A^{(1)}\right\} \subseteq\left\{B^{(1)} A^{(1)}\right\} \\
& \left\{B^{(1,2,4)} A^{(1,2,3)}\right\} \subseteq\left\{B^{(1,2,4)} A^{(1,2)}\right\} \subseteq\left\{B^{(1,4)} A^{(1,2)}\right\} \\
& \left\{B^{(1,2,4)} A^{(1,2,3)}\right\} \subseteq\left\{B^{(1,4)} A^{(1,2,3)}\right\} \subseteq\left\{B^{(1,4)} A^{(1,3)}\right\} \\
& \left\{B^{(1,2,4)} A^{(1,2,3)}\right\} \subseteq\left\{B^{(1,2)} A^{(1,2,3)}\right\} \subseteq\left\{B^{(1,2)} A^{(1,3)}\right\} \subseteq\left\{B^{(1,2)} A^{(1)}\right\} \\
& \left\{B^{(1,2,4)} A^{(1,2,3)}\right\} \subseteq\left\{B^{(1,2)} A^{(1,2)}\right\} \subseteq\left\{B^{(1)} A^{(1,2)}\right\} \\
& \left\{B^{(1,2,4)} A^{(1,2,3)}\right\} \subseteq\left\{B^{(1)} A^{(1,2,3)}\right\} \subseteq\left\{B^{(1)} A^{(1,3)}\right\}
\end{aligned}
$$

Hence, we obtain from Lemma 8 that

$$
\begin{aligned}
& \max _{B^{(1,3,4)}, A^{(1,3)}} r\left(B^{(1,3,4)} A^{(1,3)}\right) \leq \max _{B^{(1,3,4)}, A^{(1)}} r\left(B^{(1,3,4)} A^{(1)}\right) \leq \max _{B^{(1)}, A^{(1)}} r\left(B^{(1)} A^{(1)}\right), \\
& \max _{B^{(1,3,4)}, A^{(1,3)}} r\left(B^{(1,3,4)} A^{(1,3)}\right) \leq \max _{B^{(1,4)}, A^{(1,3)}} r\left(B^{(1,4)} A^{(1,3)}\right) \leq \max _{B^{(1)}, A^{(1,3)}} r\left(B^{(1)} A^{(1,3)}\right), \\
& \max _{B^{(1,3,4)}, A^{(1,3)}} r\left(B^{(1,3,4)} A^{(1,3)}\right) \leq \max _{B^{(1,3)}, A^{(1,3)}} r\left(B^{(1,3)} A^{(1,3)}\right) \leq \max _{B^{(1,3)}, A^{(1)}} r\left(B^{(1,3)} A^{(1)}\right), \\
& \max _{B^{(1,4)}, A^{(1,3,4)}} r\left(B^{(1,4)} A^{(1,3,4)}\right) \leq \max _{B^{(1)}, A^{(1,3,4)}} r\left(B^{(1)} A^{(1,3,4)}\right), \\
& \max _{B^{(1,4)}, A^{(1,3,4)}} r\left(B^{(1,4)} A^{(1,3,4)}\right) \leq \max _{B^{(1,4)}, A^{(1,4)}} r\left(B^{(1,4)} A^{(1,4)}\right) \leq \max _{B^{(1),}, A^{(1,4)}} r\left(B^{(1)} A^{(1,4)}\right), \\
& \max _{B^{(1,4)}, A^{(1,3,4)}} r\left(B^{(1,4)} A^{(1,3,4)}\right) \leq \max _{B^{(1,4)}, A^{(1)}} r\left(B^{(1,4)} A^{(1)}\right),
\end{aligned}
$$

and

$$
\begin{aligned}
& \min _{B^{(1,2,4)}, A^{(1,2,3)}} r\left(B^{(1,2,4)} A^{(1,2,3)}\right) \geq \min _{B^{(1,2,4)}, A^{(1,3)}} r\left(B^{(1,2,4)} A^{(1,3)}\right) \geq \min _{B^{(1,2,4)}, A^{(1)}} r\left(B^{(1,2,4)} A^{(1)}\right) \\
& \geq \min _{B^{(1,4)}, A^{(1)}} r\left(B^{(1,4)} A^{(1)}\right) \geq \min _{B^{(1)}, A^{(1)}} r\left(B^{(1)} A^{(1)}\right), \\
& \min _{B^{(1,2,4)}, A^{(1,2,3)}} r\left(B^{(1,2,4)} A^{(1,2,3)}\right) \geq \min _{B^{(1,2,4)}, A^{(1,2)}} r\left(B^{(1,2,4)} A^{(1,2)}\right) \\
& \geq \min _{B^{(1,4)}, A^{(1,2)}} r\left(B^{(1,4)} A^{(1,2)}\right), \\
& \min _{B^{(1,2,4)}, A^{(1,2,3)}} r\left(B^{(1,2,4)} A^{(1,2,3)}\right) \geq \min _{B^{(1,4)}, A^{(1,2,3)}} r\left(B^{(1,4)} A^{(1,2,3)}\right) \\
& \geq \min _{B^{(1,4)}, A^{(1,3)}} r\left(B^{(1,4)} A^{(1,3)}\right), \\
& \min _{B^{(1,2,4)}, A^{(1,2,3)}} r\left(B^{(1,2,4)} A^{(1,2,3)}\right) \geq \min _{B^{(1,2), A^{(1,2,3)}}} r\left(B^{(1,2)} A^{(1,2,3)}\right) \\
& \geq \min _{B^{(1,2)}, A^{(1,3)}} r\left(B^{(1,2)} A^{(1,3)}\right) \\
& \min _{B^{(1,2)}, A^{(1)}} r\left(B^{(1,2)} A^{(1)}\right), \\
& \min _{B^{(1,2,4)}, A^{(1,2,3)}} r\left(B^{(1,2,4)} A^{(1,2,3)}\right) \geq \min _{B^{(1,2)}, A^{(1,2)}} r\left(B^{(1,2)} A^{(1,2)}\right) \\
& \geq \min _{B^{(1)}, A^{(1,2)}} r\left(B^{(1)} A^{(1,2)}\right),
\end{aligned}
$$




$$
\begin{aligned}
\min _{B^{(1,2,4)}, A^{(1,2,3)}} r\left(B^{(1,2,4)} A^{(1,2,3)}\right) & \geq \min _{B^{(1), A^{(1,2,3)}}} r\left(B^{(1)} A^{(1,2,3)}\right) \\
& \geq \min _{B^{(1)}, A^{(1,3)}} r\left(B^{(1)} A^{(1,3)}\right) .
\end{aligned}
$$

Combining (3.255)-(3.260) with (3.135), (3.175), and (3.109), we obtain the following results:

$$
\begin{aligned}
& \max _{B^{(1,3,4)}, A^{(1,3)}} r\left(B^{(1,3,4)} A^{(1,3)}\right) \\
& =\max _{B^{(1,4)}, A^{(1,3,4)}} r\left(B^{(1,4)} A^{(1,3,4)}\right)=\max _{B^{(1,3,4)}, A^{(1)}} r\left(B^{(1,3,4)} A^{(1)}\right) \\
& =\max _{B^{(1,4)}, A^{(1,4)}} r\left(B^{(1,4)} A^{(1,4)}\right)=\max _{B^{(1,4)}, A^{(1,3)}} r\left(B^{(1,4)} A^{(1,3)}\right)=\max _{B^{(1,4)}, A^{(1)}} r\left(B^{(1,4)} A^{(1)}\right) \\
& =\max _{B^{(1,3)}, A^{(1,3)}} r\left(B^{(1,3)} A^{(1,3)}\right)=\max _{B^{(1,3)}, A^{(1)}} r\left(B^{(1,3)} A^{(1)}\right)=\max _{B^{(1)}, A^{(1,3,4)}} r\left(B^{(1)} A^{(1,3,4)}\right) \\
& =\max _{B^{(1)}, A^{(1,4)}} r\left(B^{(1)} A^{(1,4)}\right)=\max _{B^{(1)}, A^{(1,3)}} r\left(B^{(1)} A^{(1,3)}\right)=\max _{B^{(1)}, A^{(1)}} r\left(B^{(1)} A^{(1)}\right) \\
& =\min \{m, n, p\},
\end{aligned}
$$

establishing (3.139), (3.181), (3.183), (3.187), (3.199), (3.203), (3.223), (3.229), (3.231), (3.235), respectively. Combining (3.261)-(3.266) with (3.148) and (3.110), we obtain the following results:

$$
\begin{aligned}
& \min _{B^{(1,2,4)}, A^{(1,2,3)}} r\left(B^{(1,2,4)} A^{(1,2,3)}\right) \\
& =\min _{B^{(1,2,4)}, A^{(1,3)}} r\left(B^{(1,2,4)} A^{(1,3)}\right)=\min _{B^{(1,2,4)}, A^{(1,2)}} r\left(B^{(1,2,4)} A^{(1,2)}\right) \\
& =\min _{B^{(1,2,4)}, A^{(1)}} r\left(B^{(1,2,4)} A^{(1)}\right)=\min _{B^{(1,4)}, A^{(1,2,3)}} r\left(B^{(1,4)} A^{(1,2,3)}\right)=\min _{B^{(1,4)}, A^{(1,3)}} r\left(B^{(1,4)} A^{(1,3)}\right) \\
& =\min _{B^{(1,4)}, A^{(1,2)}} r\left(B^{(1,4)} A^{(1,2)}\right)=\min _{B^{(1,4)}, A^{(1)}} r\left(B^{(1,4)} A^{(1)}\right)=\min _{B^{(1,2)}, A^{(1,2,3)}} r\left(B^{(1,2)} A^{(1,2,3)}\right) \\
& =\min _{B^{(1,2),} A^{(1,3)}} r\left(B^{(1,2)} A^{(1,3)}\right)=\min _{B^{(1,2)}, A^{(1,2)}} r\left(B^{(1,2)} A^{(1,2)}\right)=\min _{B^{(1,2)}, A^{(1)}} r\left(B^{(1,2)} A^{(1)}\right) \\
& =\min _{B^{(1)}, A^{(1,2,3)}} r\left(B^{(1)} A^{(1,2,3)}\right)=\min _{B^{(1)}, A^{(1,3)}} r\left(B^{(1)} A^{(1,3)}\right)=\min _{B^{(1)}, A^{(1,2)}} r\left(B^{(1)} A^{(1,2)}\right) \\
& =\min _{B^{(1)}, A^{(1)}} r\left(B^{(1)} A^{(1)}\right)=\max \{0, r(A)+r(B)-n\},
\end{aligned}
$$

establishing (3.152), (3.154), (3.156), (3.180), (3.184), (3.186), (3.188), (3.212), (3.216), (3.218), (3.220), (3.228), (3.232), (3.234), and (3.236), respectively.

Applying (2.66) and (2.67) to (3.10) yields

$$
\begin{aligned}
& \max _{B^{(1,3,4)}, A^{(1,3,4)}} r\left(B^{(1,3,4)} A^{(1,3,4)}\right) \\
& =\max _{V, W} r\left[\left(B^{\dagger}+F_{B} V E_{B}\right)\left(A^{\dagger}+F_{A} W E_{A}\right)\right] \\
& =\min \left\{r\left[B^{\dagger} A^{\dagger}, B^{\dagger} F_{A}, F_{B}\right], r\left[\begin{array}{c}
B^{\dagger} A^{\dagger} \\
E_{B} A^{\dagger} \\
E_{A}
\end{array}\right], r\left[\begin{array}{cc}
B^{\dagger} A^{\dagger} & F_{B} \\
E_{A} & 0
\end{array}\right], r\left[\begin{array}{cc}
B^{\dagger} A^{\dagger} & B^{\dagger} F_{A} \\
E_{B} A^{\dagger} & E_{B} F_{A}
\end{array}\right]\right\},
\end{aligned}
$$




$$
\begin{aligned}
\min _{B^{(1,3,4)}, A^{(1,3,4)}} r\left(B^{(1,3,4)} A^{(1,3,4)}\right) & =\min _{V, W} r\left[\left(B^{\dagger}+F_{B} V E_{B}\right)\left(A^{\dagger}+F_{A} W E_{A}\right)\right] \\
& =r\left[\begin{array}{c}
B^{\dagger} A^{\dagger} \\
E_{B} A^{\dagger} \\
E_{A}
\end{array}\right]+r\left[B^{\dagger} A^{\dagger}, B^{\dagger} F_{A}, F_{B}\right]+\max \left\{s_{1}, s_{2}\right\},
\end{aligned}
$$

where

$$
\begin{aligned}
& s_{1}=r\left[\begin{array}{cc}
B^{\dagger} A^{\dagger} & F_{B} \\
E_{A} & 0
\end{array}\right]-r\left[\begin{array}{ccc}
B^{\dagger} A^{\dagger} & F_{B} & B^{\dagger} F_{A} \\
E_{A} & 0 & 0
\end{array}\right]-r\left[\begin{array}{cc}
B^{\dagger} A^{\dagger} & F_{B} \\
E_{A} & 0 \\
E_{B} A^{\dagger} & 0
\end{array}\right], \\
& s_{2}=r\left[\begin{array}{cc}
B^{\dagger} A^{\dagger} & B^{\dagger} F_{A} \\
E_{B} A^{\dagger} & E_{B} F_{A}
\end{array}\right]-r\left[\begin{array}{ccc}
B^{\dagger} A^{\dagger} & B^{\dagger} F_{A} & F_{B} \\
E_{B} A^{\dagger} & E_{B} F_{A} & 0
\end{array}\right]-r\left[\begin{array}{cc}
B^{\dagger} A^{\dagger} & B^{\dagger} F_{A} \\
E_{B} A^{\dagger} & E_{B} F_{A} \\
E_{A} & 0
\end{array}\right] .
\end{aligned}
$$

Simplifying the formulas by (2.29)-(2.31) gives

$$
\begin{aligned}
& r\left[B^{\dagger} A^{\dagger}, B^{\dagger} F_{A}, F_{B}\right]=r\left[B^{\dagger} A^{\dagger}, B^{\dagger} F_{A}\right]+r\left(F_{B}\right)=r(B)+p-r(B)=p, \\
& r\left[\begin{array}{c}
B^{\dagger} A^{\dagger} \\
E_{B} A^{\dagger} \\
E_{A}
\end{array}\right]=r\left[\begin{array}{c}
B^{\dagger} A^{\dagger} \\
E_{B} A^{\dagger}
\end{array}\right]+r\left(E_{A}\right)=r(A)+m-r(A)=m, \\
& r\left[\begin{array}{cc}
B^{\dagger} A^{\dagger} & F_{B} \\
E_{A} & 0
\end{array}\right]=r\left(B^{\dagger} A^{\dagger}\right)+r\left(E_{A}\right)+r\left(F_{B}\right)=m+p+r(A B)-r(A)-r(B), \\
& r\left[\begin{array}{ccc}
B^{\dagger} A^{\dagger} & F_{B} & B^{\dagger} F_{A} \\
E_{A} & 0 & 0
\end{array}\right]=r\left[B^{\dagger} A^{\dagger}, B^{\dagger} F_{A}\right]+r\left(E_{A}\right)+r\left(F_{B}\right)=m+p-r(A), \\
& r\left[\begin{array}{cc}
B^{\dagger} A^{\dagger} & F_{B} \\
E_{A} & 0 \\
E_{B} A^{\dagger} & 0
\end{array}\right]=r\left[\begin{array}{c}
B^{\dagger} A^{\dagger} \\
E_{B} A^{\dagger}
\end{array}\right]+r\left(E_{A}\right)+r\left(F_{B}\right)=m+p-r(B), \\
& r\left[\begin{array}{ccc}
B^{\dagger} A^{\dagger} & B^{\dagger} F_{A} & F_{B} \\
E_{B} A^{\dagger} & E_{B} F_{A} & 0
\end{array}\right]=r\left[\begin{array}{cc}
B^{\dagger} A^{\dagger} & B^{\dagger} F_{A} \\
E_{B} A^{\dagger} & E_{B} F_{A}
\end{array}\right]+r\left(F_{B}\right)=n+p-r(B), \\
& r\left[\begin{array}{cc}
B^{\dagger} A^{\dagger} & B^{\dagger} F_{A} \\
E_{B} A^{\dagger} & E_{B} F_{A} \\
E_{A} & 0
\end{array}\right]=r\left[\begin{array}{cc}
B^{\dagger} A^{\dagger} & B^{\dagger} F_{A} \\
E_{B} A^{\dagger} & E_{B} F_{A}
\end{array}\right]+r\left(E_{A}\right)=m+n-r(A) .
\end{aligned}
$$

Substituting (3.269)-(3.275) into (3.267) and (3.268) and simplifying leads to (3.127) and (3.128), respectively.

Note from (2.9) that

$$
r\left(B^{(1,3,4)} A^{(1,2,4)}\right)=r\left(B^{(1,3,4)} A^{(1,2,4)} A\right)=r\left(B^{(1,3,4)} A^{\dagger}\right)
$$

hold for all $B^{(1,3,4)}$ and $A^{(1,2,4)}$. Hence, (3.129) and (3.130) follow from (3.125) and (3.126). Equations (3.159) and (3.160) can be established by pattern symmetry. 
Note from (2.10) and (2.13) that

$$
r\left(B^{(1,3,4)} A^{(1,2,3)}\right)=r\left(B^{(1,3,4)} A^{(1,2,3)} A\right), \quad r\left(B^{(1,3,4)} A^{(1,2)}\right)=r\left(B^{(1,3,4)} A^{(1,2)} A\right)
$$

hold for all $B^{(1,3,4)}, A^{(1,2,3)}$ and $A^{(1,2)}$, where by (3.12) and (3.15),

$$
B^{(1,3,4)} A^{(1,2,3)} A=B^{(1,3,4)} A^{(1,2)} A=\left(B^{\dagger}+F_{B} V E_{B}\right)\left(A^{\dagger} A+F_{A} W A\right) .
$$

Applying (2.66) and (2.67) to the right-hand side of (3.278) yields

$$
\begin{aligned}
& \max _{V, W} r {\left[\left(B^{\dagger}+F_{B} V E_{B}\right)\left(A^{\dagger} A+F_{A} W A\right)\right] } \\
&= \min \left\{r\left[B^{\dagger} A^{\dagger} A, B^{\dagger} F_{A}, F_{B}\right], r\left[\begin{array}{c}
B^{\dagger} A^{\dagger} A \\
E_{B} A^{\dagger} A \\
A
\end{array}\right],\right. \\
&\left.r\left[\begin{array}{cc}
B^{\dagger} A^{\dagger} A & F_{B} \\
A & 0
\end{array}\right], r\left[\begin{array}{ll}
B^{\dagger} A^{\dagger} A & B^{\dagger} F_{A} \\
E_{B} A^{\dagger} A & E_{B} F_{A}
\end{array}\right]\right\}, \\
& \min _{V, W} r\left[\left(B^{\dagger}+F_{B} V E_{B}\right)\left(A^{\dagger} A+F_{A} W A\right)\right] \\
&=r\left[\begin{array}{c}
B^{\dagger} A^{\dagger} A \\
E_{B} A^{\dagger} A \\
A
\end{array}\right]+r\left[B^{\dagger} A^{\dagger} A, B^{\dagger} F_{A}, F_{B}\right]+\max \left\{s_{1}, s_{2}\right\},
\end{aligned}
$$

where

$$
\begin{aligned}
& s_{1}=r\left[\begin{array}{cc}
B^{\dagger} A^{\dagger} A & F_{B} \\
A & 0
\end{array}\right]-r\left[\begin{array}{ccc}
B^{\dagger} A^{\dagger} A & F_{B} & B^{\dagger} F_{A} \\
A & 0 & 0
\end{array}\right]-r\left[\begin{array}{cc}
B^{\dagger} A^{\dagger} A & F_{B} \\
A & 0 \\
E_{B} A^{\dagger} A & 0
\end{array}\right], \\
& s_{2}=r\left[\begin{array}{cc}
B^{\dagger} A^{\dagger} A & B^{\dagger} F_{A} \\
E_{B} A^{\dagger} A & E_{B} F_{A}
\end{array}\right]-r\left[\begin{array}{ccc}
B^{\dagger} A^{\dagger} A & B^{\dagger} F_{A} & F_{B} \\
E_{B} A^{\dagger} A & E_{B} F_{A} & 0
\end{array}\right]-r\left[\begin{array}{cc}
B^{\dagger} A^{\dagger} A & B^{\dagger} F_{A} \\
E_{B} A^{\dagger} A & E_{B} F_{A} \\
A & 0
\end{array}\right] .
\end{aligned}
$$

Simplifying the formulas by (2.29)-(2.31) gives

$$
\begin{aligned}
& r\left[B^{\dagger} A^{\dagger} A, B^{\dagger} F_{A}, F_{B}\right]=r\left[B^{\dagger} A^{\dagger}, B^{\dagger} F_{A}\right]+r\left(F_{B}\right)=r(B)+p-r(B)=p, \\
& r\left[\begin{array}{c}
B^{\dagger} A^{\dagger} A \\
E_{B} A^{\dagger} A \\
A
\end{array}\right]=r(A), \quad r\left[\begin{array}{cc}
B^{\dagger} A^{\dagger} A & F_{B} \\
A & 0
\end{array}\right]=r(A)+r\left(F_{B}\right)=p+r(A)-r(B), \\
& r\left[\begin{array}{cc}
B^{\dagger} A^{\dagger} A & B^{\dagger} F_{A} \\
E_{B} A^{\dagger} A & E_{B} F_{A}
\end{array}\right]=r\left[\begin{array}{cc}
B^{\dagger} A^{\dagger} & B^{\dagger} F_{A} \\
E_{B} A^{\dagger} & E_{B} F_{A}
\end{array}\right]=n, \\
& r\left[\begin{array}{ccc}
B^{\dagger} A^{\dagger} A & F_{B} & B^{\dagger} F_{A} \\
A & 0 & 0
\end{array}\right]=r(A)+r\left(F_{B}\right)+r\left(B^{\dagger} F_{A}\right)=p+r\left[A^{*}, B\right]-r(B),
\end{aligned}
$$




$$
\begin{aligned}
& r\left[\begin{array}{cc}
B^{\dagger} A^{\dagger} A & F_{B} \\
A & 0 \\
E_{B} A^{\dagger} A & 0
\end{array}\right]=r(A)+r\left(F_{B}\right)=p+r(A)-r(B), \\
& r\left[\begin{array}{ccc}
B^{\dagger} A^{\dagger} A & B^{\dagger} F_{A} & F_{B} \\
E_{B} A^{\dagger} A & E_{B} F_{A} & 0
\end{array}\right]=r\left[\begin{array}{cc}
B^{\dagger} A^{\dagger} & B^{\dagger} F_{A} \\
E_{B} A^{\dagger} & E_{B} F_{A}
\end{array}\right]+r\left(F_{B}\right)=n+p-r(B), \\
& r\left[\begin{array}{cc}
B^{\dagger} A^{\dagger} A & B^{\dagger} F_{A} \\
E_{B} A^{\dagger} A & E_{B} F_{A} \\
A & 0
\end{array}\right]=r(A)+r\left[\begin{array}{l}
B^{\dagger} F_{A} \\
E_{B} F_{A}
\end{array}\right]=r(A)+r\left(F_{A}\right)=n .
\end{aligned}
$$

Substituting (3.281)-(3.287) into (3.279) and (3.280) and combining them with (3.277) and (3.278) lead to (3.131), (3.132), (3.137), and (3.138), respectively. Equations (3.143), (3.144), (3.207), and (3.208) can be established by pattern symmetry.

Applying (2.66) and (2.67) to the right-hand side of (3.13) yields

$$
\begin{aligned}
& \max _{B^{(1,3,4)}, A^{(1,4)}} r\left(B^{(1,3,4)} A^{(1,4)}\right) \\
&=\max _{V, W} r\left[\left(B^{\dagger}+F_{B} V E_{B}\right)\left(A^{\dagger}+W E_{A}\right)\right] \\
&=\min \left\{r\left[B^{\dagger} A^{\dagger}, B^{\dagger}, F_{B}\right], r\left[\begin{array}{c}
B^{\dagger} A^{\dagger} \\
E_{B} A^{\dagger} \\
E_{A}
\end{array}\right], r\left[\begin{array}{cc}
B^{\dagger} A^{\dagger} & F_{B} \\
E_{A} & 0
\end{array}\right], r\left[\begin{array}{cc}
B^{\dagger} A^{\dagger} & B^{\dagger} \\
E_{B} A^{\dagger} & E_{B}
\end{array}\right]\right\}, \\
& \min _{B^{(1,3,4)}, A^{(1,4)}} r\left(B^{(1,3,4)} A^{(1,4)}\right)=\min _{V, W} r\left[\left(B^{\dagger}+F_{B} V E_{B}\right)\left(A^{\dagger}+W E_{A}\right)\right] \\
&=r\left[\begin{array}{c}
B^{\dagger} A^{\dagger} \\
E_{B} A^{\dagger} \\
E_{A}
\end{array}\right]+r\left[B^{\dagger} A^{\dagger}, B^{\dagger}, F_{B}\right]+\max \left\{s_{1}, s_{2}\right\},
\end{aligned}
$$

where

$$
\begin{gathered}
s_{1}=r\left[\begin{array}{cc}
B^{\dagger} A^{\dagger} & F_{B} \\
E_{A} & 0
\end{array}\right]-r\left[\begin{array}{ccc}
B^{\dagger} A^{\dagger} & F_{B} & B^{\dagger} \\
E_{A} & 0 & 0
\end{array}\right]-r\left[\begin{array}{cc}
B^{\dagger} A^{\dagger} & F_{B} \\
E_{A} & 0 \\
E_{B} A^{\dagger} & 0
\end{array}\right], \\
s_{2}=r\left[\begin{array}{cc}
B^{\dagger} A^{\dagger} & B^{\dagger} \\
E_{B} A^{\dagger} & E_{B}
\end{array}\right]-r\left[\begin{array}{ccc}
B^{\dagger} A^{\dagger} & B^{\dagger} & F_{B} \\
E_{B} A^{\dagger} & E_{B} & 0
\end{array}\right]-r\left[\begin{array}{cc}
B^{\dagger} A^{\dagger} & B^{\dagger} \\
E_{B} A^{\dagger} & E_{B} \\
E_{A} & 0
\end{array}\right] .
\end{gathered}
$$

Simplifying the formulas by (2.29)-(2.31) gives

$$
\begin{aligned}
& r\left[B^{\dagger} A^{\dagger}, B^{\dagger}, F_{B}\right]=r\left[B^{\dagger}, F_{B}\right]=p, \quad r\left[\begin{array}{c}
B^{\dagger} A^{\dagger} \\
E_{B} A^{\dagger} \\
E_{A}
\end{array}\right]=m, \\
& r\left[\begin{array}{cc}
B^{\dagger} A^{\dagger} & F_{B} \\
E_{A} & 0
\end{array}\right]=m+p+r(A B)-r(A)-r(B), \\
& r\left[\begin{array}{cc}
B^{\dagger} A^{\dagger} & B^{\dagger} \\
E_{B} A^{\dagger} & E_{B}
\end{array}\right]=r\left[\begin{array}{l}
B^{\dagger} \\
E_{B}
\end{array}\right]=n,
\end{aligned}
$$




$$
\begin{aligned}
& r\left[\begin{array}{ccc}
B^{\dagger} A^{\dagger} & F_{B} & B^{\dagger} \\
E_{A} & 0 & 0
\end{array}\right]=m+p-r(A), \\
& r\left[\begin{array}{cc}
B^{\dagger} A^{\dagger} & F_{B} \\
E_{A} & 0 \\
E_{B} A^{\dagger} & 0
\end{array}\right]=m+p-r(B), \\
& r\left[\begin{array}{ccc}
B^{\dagger} A^{\dagger} & B^{\dagger} & F_{B} \\
E_{B} A^{\dagger} & E_{B} & 0
\end{array}\right]=r\left[\begin{array}{cc}
B^{\dagger} & F_{B} \\
E_{B} & 0
\end{array}\right]=r(B)+r\left(E_{B}\right)+r\left(F_{B}\right)=n+p-r(B), \\
& r\left[\begin{array}{cc}
B^{\dagger} A^{\dagger} & B^{\dagger} \\
E_{B} A^{\dagger} & E_{B} \\
E_{A} & 0
\end{array}\right]=r\left[\begin{array}{l}
B^{\dagger} \\
E_{B}
\end{array}\right]+r\left(E_{A}\right)=m+n-r(A) .
\end{aligned}
$$

Substituting (3.290)-(3.294) into (3.288) and (3.289) leads to (3.133) and (3.134), respectively. Equations (3.191) and (3.192) can be established by pattern symmetry.

Applying (2.38) and (2.39) gives

$$
\min _{B^{(1,3,4)}} r\left(B^{(1,3,4)} A^{(1)}\right)=r\left(B^{*} A^{(1)}\right), \quad \min _{A^{(1)}} r\left(B^{*} A^{(1)}\right)=r(A)+r(B)-r\left[A^{*}, B\right] .
$$

Combining the two equalities in (3.295) yields

$$
\min _{B^{(1,3,4)}, A^{(1)}} r\left(B^{(1,3,4)} A^{(1)}\right)=r(A)+r(B)-r\left[A^{*}, B\right]
$$

as required for (3.140). Equation (3.224) can be established by pattern symmetry.

Note from (2.9) and (2.13) that

$$
r\left(B^{(1,2,4)} A^{(1,2,4)}\right)=r\left(B^{(1,2,4)} A^{(1,2,4)} A\right)=r\left(B^{(1,2,4)} A^{\dagger} A\right)=r\left(B^{(1,2,4)} A^{*}\right)
$$

hold for all $B^{(1,2,4)}$ and $A^{(1,2,4)}$. Applying (2.40) and (2.41) to (3.296) and simplifying, we obtain

$$
\begin{aligned}
\max _{B^{(1,2,4)}, A^{(1,2,4)}} r\left(B^{(1,2,4)} A^{(1,2,4)}\right) & =\max _{B^{(1,2,4)}} r\left(B^{(1,2,4)} A^{*}\right)=\min \{r(A), r(B)\}, \\
\min _{B^{(1,2,4)}, A^{(1,2,4)}} r\left(B^{(1,2,4)} A^{(1,2,4)}\right) & =\min _{B^{(1,2,4)}} r\left(B^{(1,2,4)} A^{*}\right)=r(A)+r(B)-r\left[A^{*}, B\right],
\end{aligned}
$$

thus establishing (3.145) and (3.146). Equations (3.163) and (3.164) can be established by pattern symmetry.

Note from (2.9) and (2.13) that

$$
r\left(B^{(1,2,4)} A^{(1,4)}\right)=r\left(B B^{(1,2,4)} A^{(1,4)}\right)=r\left(B^{(1,2)} A^{(1,4)}\right)=r\left(B B^{(1,2)} A^{(1,4)}\right)
$$

hold for all $B^{(1,2,4)}$ and $B^{(1,2)}$, where by (3.53),

$$
B B^{(1,2)} A^{(1,4)}=\left(B B^{\dagger}+B V E_{B}\right)\left(A^{\dagger}+W E_{A}\right) .
$$

Applying (2.66) and (2.67) to the right-hand side of (3.298) and simplifying, we obtain 


$$
\begin{aligned}
& \max _{V, W} r\left[\left(B B^{\dagger}+B V E_{B}\right)\left(A^{\dagger}+W E_{A}\right)\right] \\
& \quad=\min \left\{r\left[B B^{\dagger} A^{\dagger}, B B^{\dagger}, B\right], r\left[\begin{array}{c}
B B^{\dagger} A^{\dagger} \\
E_{B} A^{\dagger} \\
E_{A}
\end{array}\right], r\left[\begin{array}{cc}
B B^{\dagger} A^{\dagger} & B \\
E_{A} & 0
\end{array}\right], r\left[\begin{array}{cc}
B B^{\dagger} A^{\dagger} & B B^{\dagger} \\
E_{B} A^{\dagger} & E_{B}
\end{array}\right]\right\} \\
& \min _{V, W} r\left[\left(B B^{\dagger}+B V E_{B}\right)\left(A^{\dagger}+W E_{A}\right)\right] \\
& \quad=r\left[\begin{array}{c}
B B^{\dagger} A^{\dagger} \\
E_{B} A^{\dagger} \\
E_{A}
\end{array}\right]+r\left[B B^{\dagger} A^{\dagger}, B B^{\dagger}, B\right]+\max \left\{s_{1}, s_{2}\right\},
\end{aligned}
$$

where

$$
\begin{aligned}
& s_{1}=r\left[\begin{array}{cc}
B B^{\dagger} A^{\dagger} & B \\
E_{A} & 0
\end{array}\right]-r\left[\begin{array}{ccc}
B B^{\dagger} A^{\dagger} & B & B B^{\dagger} \\
E_{A} & 0 & 0
\end{array}\right]-r\left[\begin{array}{cc}
B B^{\dagger} A^{\dagger} & B \\
E_{A} & 0 \\
E_{B} A^{\dagger} & 0
\end{array}\right], \\
& s_{2}=r\left[\begin{array}{cc}
B B^{\dagger} A^{\dagger} & B B^{\dagger} \\
E_{B} A^{\dagger} & E_{B}
\end{array}\right]-r\left[\begin{array}{ccc}
B B^{\dagger} A^{\dagger} & B B^{\dagger} & B \\
E_{B} A^{\dagger} & E_{B} & 0
\end{array}\right]-r\left[\begin{array}{cc}
B B^{\dagger} A^{\dagger} & B B^{\dagger} \\
E_{B} A^{\dagger} & E_{B} \\
E_{A} & 0
\end{array}\right] .
\end{aligned}
$$

Simplifying the formulas by (2.29)-(2.31) gives

$$
\begin{aligned}
& r\left[B B^{\dagger} A^{\dagger}, B B^{\dagger}, B\right]=r(B), \quad r\left[\begin{array}{c}
B B^{\dagger} A^{\dagger} \\
E_{B} A^{\dagger} \\
E_{A}
\end{array}\right]=m, \\
& r\left[\begin{array}{cc}
B B^{\dagger} A^{\dagger} & B \\
E_{A} & 0
\end{array}\right]=m-r(A)+r(B), \quad r\left[\begin{array}{cc}
B B^{\dagger} A^{\dagger} & B B^{\dagger} \\
E_{B} A^{\dagger} & E_{B}
\end{array}\right]=n, \\
& r\left[\begin{array}{ccc}
B B^{\dagger} A^{\dagger} & B & B B^{\dagger} \\
E_{A} & 0 & 0
\end{array}\right]=r(B)+r\left(E_{A}\right)=m-r(A)+r(B), \\
& r\left[\begin{array}{cc}
B B^{\dagger} A^{\dagger} & B \\
E_{A} & 0 \\
E_{B} A^{\dagger} & 0
\end{array}\right]=r\left(E_{A}\right)+r(B)+r\left(E_{B} A^{\dagger}\right)=m+r\left[A^{*}, B\right]-r(A), \\
& r\left[\begin{array}{ccc}
B B^{\dagger} A^{\dagger} & B B^{\dagger} & B \\
E_{B} A^{\dagger} & E_{B} & 0
\end{array}\right]=r(B)+r\left(E_{B}\right)=n, \\
& r\left[\begin{array}{cc}
B B^{\dagger} A^{\dagger} & B B^{\dagger} \\
E_{B} A^{\dagger} & E_{B} \\
E_{A} & 0
\end{array}\right]=r\left(E_{A}\right)+r\left[\begin{array}{c}
B B^{\dagger} \\
E_{B}
\end{array}\right]=m+n-r(A) .
\end{aligned}
$$

Substituting (3.301)-(3.306) into (3.299) and (3.300) and combining them with (3.297) and (3.298) lead to (3.149), (3.150), (3.213) and (3.214), respectively. Equations (3.195), (3.196), (3.201) and (3.202) can be established by pattern symmetry. 
Note from (2.9) and (2.13) that

$$
r\left(B^{(1,2,4)} A^{(1,3)}\right)=r\left(B B^{(1,2,4)} A^{(1,3)}\right)=r\left(B^{(1,2)} A^{(1,3)}\right)=r\left(B B^{(1,2)} A^{(1,3)}\right)
$$

hold for all $B^{(1,2,4)}, B^{(1,2)}$, and $A^{(1,3)}$, where by (3.54),

$$
B B^{(1,2)} A^{(1,3)}=\left(B B^{\dagger}+B V E_{B}\right)\left(A^{\dagger}+F_{A} W\right) .
$$

Applying (2.66) to the right-hand side of (3.308) and simplifying, we obtain

$$
\begin{aligned}
& \max _{V, W} r\left[\left(B B^{\dagger}+B V E_{B}\right)\left(A^{\dagger}+F_{A} W\right)\right] \\
& \quad=\min \left\{r\left[B B^{\dagger} A^{\dagger}, B B^{\dagger} F_{A}, B\right], r\left[\begin{array}{c}
B B^{\dagger} A^{\dagger} \\
E_{B} A^{\dagger} \\
I_{m}
\end{array}\right], r\left[\begin{array}{cc}
B B^{\dagger} A^{\dagger} & B \\
I_{m} & 0
\end{array}\right], r\left[\begin{array}{cc}
B B^{\dagger} A^{\dagger} & B B^{\dagger} F_{A} \\
E_{B} A^{\dagger} & E_{B} F_{A}
\end{array}\right]\right\} \\
& \quad=\min \{r(B), m, m+r(B), n\}=\min \{m, r(B)\} .
\end{aligned}
$$

Combining (3.307), (3.308), and (3.309) yields (3.151) and (3.215). Equations (3.179) and (3.185) can be established by pattern symmetry.

Note from (2.9) and (2.13) that

$$
r\left(B^{(1,2,4)} A^{(1)}\right)=r\left(B B^{(1,2,4)} A^{(1)}\right)=r\left(B^{(1,2)} A^{(1)}\right)=r\left(B B^{(1,2)} A^{(1)}\right)
$$

hold for all $B^{(1,2,4)}, B^{(1,2)}$, and $A^{(1)}$. Applying (2.48) to $B^{(1,2)} A^{(1)}$ and simplifying, we obtain

$$
\begin{aligned}
& \max _{B^{(1,2,4)}} r\left(B^{(1,2,4)} A^{(1)}\right)=\max _{B^{(1,2)}} r\left(B^{(1,2)} A^{(1)}\right)=\min \left\{r\left(A^{(1)}\right), r(B)\right\}, \\
& \max _{A^{(1)}} r\left(A^{(1)}\right)=\min \{m, n\} .
\end{aligned}
$$

Combining the two equalities in (3.311) and (3.312) yields

$$
\max _{B^{(1,2,4)}, A^{(1)}} r\left(B^{(1,2,4)} A^{(1)}\right)=\max _{B^{(1,2)}, A^{(1)}} r\left(B^{(1,2)} A^{(1)}\right)=\min \{m, r(B)\}
$$

as required for (3.155) and (3.219). Equations (3.227) and (3.233) can be established by pattern symmetry.

Note from (2.8) and (2.10), (2.36), and (2.37) that

$$
\begin{aligned}
& r\left(B^{(1,2,3)} A^{(1,2,4)}\right)=r\left(B B^{(1,2,3)} A^{(1,2,4)} A\right)=r\left(B B^{\dagger} A^{\dagger} A\right)=r(A B), \\
& r\left(B^{(1,2,3)} A^{(1,2,3)}\right)=r\left(B B^{(1,2,3)} A^{(1,2,3)}\right)=r\left(B B^{\dagger} A^{(1,2,3)}\right)=r\left(B^{\dagger} A^{(1,2,3)}\right), \\
& r\left(B^{(1,2,3)} A^{(1,4)}\right)=r\left(B B^{(1,2,3)} A^{(1,4)}\right)=r\left(B B^{\dagger} A^{(1,4)}\right)=r\left(B^{\dagger} A^{(1,4)}\right), \\
& r\left(B^{(1,2,3)} A^{(1,3)}\right)=r\left(B B^{(1,2,3)} A^{(1,3)}\right)=r\left(B B^{\dagger} A^{(1,3)}\right)=r\left(B^{\dagger} A^{(1,3)}\right), \\
& r\left(B^{(1,2,3)} A^{(1,2)}\right)=r\left(B B^{(1,2,3)} A^{(1,2)}\right)=r\left(B B^{\dagger} A^{(1,2)}\right)=r\left(B^{\dagger} A^{(1,2)}\right), \\
& r\left(B^{(1,2,3)} A^{(1)}\right)=r\left(B B^{(1,2,3)} A^{(1)}\right)=r\left(B B^{\dagger} A^{(1)}\right)=r\left(B^{\dagger} A^{(1)}\right) .
\end{aligned}
$$


Thus (3.161)-(3.172) follow from (3.113)-(3.124). Equations (3.177), (3.178), (3.193), (3.194), (3.209), (3.210), (3.225), (3.226) can be established by pattern symmetry.

Applying (2.67) to (3.37) and simplifying, we obtain

$$
\begin{aligned}
\min _{B^{(1,4)}, A^{(1,4)}} r\left(B^{(1,4)} A^{(1,4)}\right) & =\min _{V, W} r\left[\left(B^{\dagger}+V E_{B}\right)\left(A^{\dagger}+W E_{A}\right)\right] \\
& =r\left[\begin{array}{c}
B^{\dagger} A^{\dagger} \\
E_{B} A^{\dagger} \\
E_{A}
\end{array}\right]+r\left[B^{\dagger} A^{\dagger}, B^{\dagger}, I_{p}\right]+\max \left\{s_{1}, s_{2}\right\},
\end{aligned}
$$

where

$$
\begin{gathered}
s_{1}=r\left[\begin{array}{cc}
B^{\dagger} A^{\dagger} & I_{p} \\
E_{A} & 0
\end{array}\right]-r\left[\begin{array}{ccc}
B^{\dagger} A^{\dagger} & I_{p} & B^{\dagger} \\
E_{A} & 0 & 0
\end{array}\right]-r\left[\begin{array}{cc}
B^{\dagger} A^{\dagger} & I_{p} \\
E_{A} & 0 \\
E_{B} A^{\dagger} & 0
\end{array}\right], \\
s_{2}=r\left[\begin{array}{ll}
B^{\dagger} A^{\dagger} & B^{\dagger} \\
E_{B} A^{\dagger} & E_{B}
\end{array}\right]-r\left[\begin{array}{ccc}
B^{\dagger} A^{\dagger} & B^{\dagger} & I_{p} \\
E_{B} A^{\dagger} & E_{B} & 0
\end{array}\right]-r\left[\begin{array}{cc}
B^{\dagger} A^{\dagger} & B^{\dagger} \\
E_{B} A^{\dagger} & E_{B} \\
E_{A} & 0
\end{array}\right] .
\end{gathered}
$$

Simplifying the formulas by (2.29)-(2.31) gives

$$
\begin{aligned}
& r\left[B^{\dagger} A^{\dagger}, B^{\dagger}, I_{p}\right]=p, \quad r\left[\begin{array}{c}
B^{\dagger} A^{\dagger} \\
E_{B} A^{\dagger} \\
E_{A}
\end{array}\right]=m, \quad r\left[\begin{array}{cc}
B^{\dagger} A^{\dagger} & I_{p} \\
E_{A} & 0
\end{array}\right]=m+p-r(A), \\
& r\left[\begin{array}{cc}
B^{\dagger} A^{\dagger} & B^{\dagger} \\
E_{B} A^{\dagger} & E_{B}
\end{array}\right]=n, \quad r\left[\begin{array}{ccc}
B^{\dagger} A^{\dagger} & I_{p} & B^{\dagger} \\
E_{A} & 0 & 0
\end{array}\right]=p+r\left(E_{A}\right)=m+p-r(A), \\
& r\left[\begin{array}{cc}
B^{\dagger} A^{\dagger} & I_{p} \\
E_{A} & 0 \\
E_{B} A^{\dagger} & 0
\end{array}\right]=p+r\left(E_{A}\right)+r\left(E_{B} A^{\dagger}\right)=m+p+r\left[A^{*}, B\right]-r(A)-r(B), \\
& r\left[\begin{array}{ccc}
B^{\dagger} A^{\dagger} & B^{\dagger} & I_{p} \\
E_{B} A^{\dagger} & E_{B} & 0
\end{array}\right]=p+r\left(E_{B}\right)=n+p-r(B), \\
& r\left[\begin{array}{cc}
B^{\dagger} A^{\dagger} & B^{\dagger} \\
E_{B} A^{\dagger} & E_{B} \\
E_{A} & 0
\end{array}\right]=m+n-r(A) \text {. }
\end{aligned}
$$

Substituting (3.314)-(3.318) into (3.313) lead to (3.182). Equation (3.200) can be established by pattern symmetry.

Applying (2.66) and (2.67) to (3.45) and simplifying, we obtain

$$
\begin{aligned}
& \max _{B^{(1,3)}, A^{(1,4)}} r\left(B^{(1,3)} A^{(1,4)}\right) \\
& \quad=\max _{V, W} r\left[\left(B^{\dagger}+F_{B} V\right)\left(A^{\dagger}+W E_{A}\right)\right]
\end{aligned}
$$




$$
\begin{gathered}
=\min \left\{r\left[B^{\dagger} A^{\dagger}, B^{\dagger}, F_{B}\right], r\left[\begin{array}{c}
B^{\dagger} A^{\dagger} \\
A^{\dagger} \\
E_{A}
\end{array}\right], r\left[\begin{array}{cc}
B^{\dagger} A^{\dagger} & F_{B} \\
E_{A} & 0
\end{array}\right], r\left[\begin{array}{cc}
B^{\dagger} A^{\dagger} & B^{\dagger} \\
A^{\dagger} & I_{n}
\end{array}\right]\right\}, \\
\min _{B^{(1,3), A^{(1,4)}} r\left(B^{(1,3)} A^{(1,4)}\right)}=\min _{V, W} r\left[\left(B^{\dagger}+F_{B} V\right)\left(A^{\dagger}+W E_{A}\right)\right] \\
=r\left[\begin{array}{c}
B^{\dagger} A^{\dagger} \\
A^{\dagger} \\
E_{A}
\end{array}\right]+r\left[B^{\dagger} A^{\dagger}, B^{\dagger}, F_{B}\right]+\max \left\{s_{1}, s_{2}\right\},
\end{gathered}
$$

where

$$
\begin{aligned}
& s_{1}=r\left[\begin{array}{cc}
B^{\dagger} A^{\dagger} & F_{B} \\
E_{A} & 0
\end{array}\right]-r\left[\begin{array}{ccc}
B^{\dagger} A^{\dagger} & F_{B} & B^{\dagger} \\
E_{A} & 0 & 0
\end{array}\right]-r\left[\begin{array}{cc}
B^{\dagger} A^{\dagger} & F_{B} \\
E_{A} & 0 \\
A^{\dagger} & 0
\end{array}\right], \\
& s_{2}=r\left[\begin{array}{cc}
B^{\dagger} A^{\dagger} & B^{\dagger} \\
A^{\dagger} & I_{n}
\end{array}\right]-r\left[\begin{array}{ccc}
B^{\dagger} A^{\dagger} & B^{\dagger} & F_{B} \\
A^{\dagger} & I_{n} & 0
\end{array}\right]-r\left[\begin{array}{cc}
B^{\dagger} A^{\dagger} & B^{\dagger} \\
A^{\dagger} & I_{n} \\
E_{A} & 0
\end{array}\right] .
\end{aligned}
$$

Simplifying the formulas by (2.29)-(2.31) gives

$$
\begin{aligned}
& r\left[B^{\dagger} A^{\dagger}, B^{\dagger}, F_{B}\right]=p, \quad r\left[\begin{array}{c}
B^{\dagger} A^{\dagger} \\
A^{\dagger} \\
E_{A}
\end{array}\right]=m, \\
& r\left[\begin{array}{cc}
B^{\dagger} A^{\dagger} & F_{B} \\
E_{A} & 0
\end{array}\right]=m+p+r(A B)-r(A)-r(B), \\
& r\left[\begin{array}{cc}
B^{\dagger} A^{\dagger} & B^{\dagger} \\
A^{\dagger} & I_{n}
\end{array}\right]=n, \quad r\left[\begin{array}{ccc}
B^{\dagger} A^{\dagger} & F_{B} & B^{\dagger} \\
E_{A} & 0 & 0
\end{array}\right]=m+p-r(A), \\
& r\left[\begin{array}{cc}
B^{\dagger} A^{\dagger} & F_{B} \\
E_{A} & 0 \\
A^{\dagger} & 0
\end{array}\right]=m+p-r(B), \\
& r\left[\begin{array}{ccc}
B^{\dagger} A^{\dagger} & B^{\dagger} & F_{B} \\
A^{\dagger} & I_{n} & 0
\end{array}\right]=n+p-r(B), \quad r\left[\begin{array}{cc}
B^{\dagger} A^{\dagger} & B^{\dagger} \\
A^{\dagger} & I_{n} \\
E_{A} & 0
\end{array}\right]=m+n-r(A) .
\end{aligned}
$$

Substituting (3.321)-(3.325) into (3.319) and (3.320) leads to (3.197) and (3.198). Applying (2.47) and (2.65) to $B^{(1,3)} A^{(1)}$ gives

$$
\min _{B^{(1,3)}} r\left(B^{(1,3)} A^{(1)}\right)=r\left(B^{*} A^{(1)}\right), \quad \min _{A^{(1)}} r\left(B^{*} A^{(1)}\right)=r(A)+r(B)-r\left[A^{*}, B\right] .
$$

Combining the two equalities in (3.326) yields

$$
\min _{B^{(1,3)}, A^{(1)}} r\left(B^{(1,3)} A^{(1)}\right)=r(A)+r(B)-r\left[A^{*}, B\right],
$$

as required for (3.204). Equation (3.230) can be established by pattern symmetry. 
Theorem 1 shows that there do exist analytical formulas for calculating the global maximum and minimum ranks of the matrix-valued functions in (3.2)-(3.64) even it is hard to believe this work can completely be finished at the very beginning. In fact, the present author made sufficient preparations for the matrix tricks and tools used in the above proofs since the 1980s, while a systematic theory of matrix rank formulas and generalized inverses of matrices were well developed during this approach.

Many rank equalities and inequalities for the ranks of the products $B^{(i, \ldots, j)} A^{(i, \ldots, j)}$ can be established from the previous theorem. In particular, we can directly obtain the difference of the maximum and minimum ranks of $B^{(i, \ldots, j)} A^{(i, \ldots, j)}$, called the spreads of the ranks of the products $B^{(i, \ldots, j)} A^{(i, \ldots, j)}$ and their rank invariance.

Corollary 1 Let $A \in \mathbb{C}^{m \times n}$ and $B \in \mathbb{C}^{n \times p}$ be given with $m \neq 0, n \neq 0$, and $p \neq 0$, and let $M=\left[A^{*}, B\right]$. Then the following results hold.

(1) The spread of the rank of $B^{\dagger} A^{(1,3,4)}$ is

$$
\max _{A^{(1,3,4)}} r\left(B^{\dagger} A^{(1,3,4)}\right)-\min _{A^{(1,3,4)}} r\left(B^{\dagger} A^{(1,3,4)}\right)=\min \{r(B)-r(A B), m-r(A)\} .
$$

Hence, the following two statements are equivalent:

(i) $r\left(B^{\dagger} A^{(1,3,4)}\right)=r(A B)$ holds for all $A^{(1,3,4)}$.

(ii) $r(A B)=r(B)$ or $r(A)=m$.

(2) $r\left(B^{\dagger} A^{(1,2,4)}\right)=r(A B)$ holds for all $A^{(1,2,4)}$.

(3) The spread of the rank of $B^{\dagger} A^{(1,2,3)}$ is

$$
\max _{A^{(1,2,3)}} r\left(B^{\dagger} A^{(1,2,3)}\right)-\min _{A^{(1,2,3)}} r\left(B^{\dagger} A^{(1,2,3)}\right)=\min \{r(M)-r(A), r(M)-r(B)\} .
$$

Hence, the following two statements are equivalent:

(i) $r\left(B^{\dagger} A^{(1,2,3)}\right)=r(A B)$ holds for all $A^{(1,2,3)}$.

(ii) $\mathscr{R}\left(A^{*}\right) \subseteq \mathscr{R}(B)$ or $\mathscr{R}\left(A^{*}\right) \supseteq \mathscr{R}(B)$.

(4) The spread of the rank of $B^{\dagger} A^{(1,4)}$ is

$$
\max _{A^{(1,4)}} r\left(B^{\dagger} A^{(1,4)}\right)-\min _{A^{(1,4)}} r\left(B^{\dagger} A^{(1,4)}\right)=\min \{r(B)-r(A B), m-r(A)\} .
$$

Hence, the following two statements are equivalent:

(i) $r\left(B^{\dagger} A^{(1,4)}\right)=r(A B)$ holds for all $A^{(1,4)}$.

(ii) $r(A B)=r(B)$ or $r(A)=m$.

(5) The spread of the rank of $B^{\dagger} A^{(1,3)}$ is

$$
\max _{A^{(1,3)}} r\left(B^{\dagger} A^{(1,3)}\right)-\min _{A^{(1,3)}} r\left(B^{\dagger} A^{(1,3)}\right)=\min \{m-r(A)-r(B)+r(M), r(M)-r(A)\} .
$$

Hence, the following two statements are equivalent:

(i) $r\left(B^{\dagger} A^{(1,3)}\right)=r(A B)$ holds for all $A^{(1,3)}$.

(ii) $\mathscr{R}\left(A^{*}\right) \supseteq \mathscr{R}(B)$ or $r(M)=r(A)+r(B)-m$.

(6) The spread of the rank of $B^{\dagger} A^{(1,2)}$ is

$$
\max _{A^{(1,2)}} r\left(B^{\dagger} A^{(1,2)}\right)-\min _{A^{(1,2)}} r\left(B^{\dagger} A^{(1,2)}\right)=\min \{r(M)-r(A), r(M)-r(B)\} .
$$


Hence, the following two statements are equivalent:

(i) $r\left(B^{\dagger} A^{(1,2)}\right)=r(A B)$ holds for all $A^{(1,2)}$.

(ii) $\mathscr{R}\left(A^{*}\right) \subseteq \mathscr{R}(B)$ or $\mathscr{R}\left(A^{*}\right) \supseteq \mathscr{R}(B)$.

(7) The spread of the rank of $B^{\dagger} A^{(1)}$ is

$$
\max _{A^{(1)}} r\left(B^{\dagger} A^{(1)}\right)-\min _{A^{(1)}} r\left(B^{\dagger} A^{(1)}\right)=\min \{m-r(A)-r(B)+r(M), r(M)-r(A)\} .
$$

Hence, the following two statements are equivalent:

(i) $r\left(B^{\dagger} A^{(1)}\right)=r(A B)$ holds for all $A^{(1)}$.

(ii) $r(M)=r(A)+r(B)-m$ or $\mathscr{R}\left(A^{*}\right) \supseteq \mathscr{R}(B)$.

(8) The spread of the rank of $B^{(1,3,4)} A^{\dagger}$ is

$$
\max _{B^{(1,3,4)}} r\left(B^{(1,3,4)} A^{\dagger}\right)-\min _{B^{(1,3,4)}} r\left(B^{(1,3,4)} A^{\dagger}\right)=\min \{r(A)-r(A B), p-r(B)\} .
$$

Hence, the following two statements are equivalent:

(i) $r\left(B^{(1,3,4)} A^{\dagger}\right)=r(A B)$ holds for all $B^{(1,3,4)}$.

(ii) $r(A B)=r(A)$ or $r(B)=p$.

(9) The spread of the rank of $B^{(1,3,4)} A^{(1,3,4)}$ is

$$
\begin{aligned}
& \max _{B^{(1,3,4)}, A^{(1,3,4)}} r\left(B^{(1,3,4)} A^{(1,3,4)}\right)-\min _{B^{(1,3,4)}, A^{(1,3,4)}} r\left(B^{(1,3,4)} A^{(1,3,4)}\right) \\
& =\min \{m-r(A B), n-r(A B), p-r(A B), m+p-r(A)-r(B)\} .
\end{aligned}
$$

Hence, the following two statements are equivalent:

(i) $r\left(B^{(1,3,4)} A^{(1,3,4)}\right)=r(A B)$ holds for all $B^{(1,3,4)}$ and $A^{(1,3,4)}$.

(ii) $r(A B)=m$ or $r(A B)=n$ or $r(A B)=p$ or $r(A)+r(B)=m+p$.

(10) The spread of the rank of $B^{(1,3,4)} A^{(1,2,4)}$ is

$$
\begin{aligned}
& \max _{B^{(1,3,4)}, A^{(1,2,4)}} r\left(B^{(1,3,4)} A^{(1,2,4)}\right)-\min _{B^{(1,3,4)}, A^{(1,2,4)}} r\left(B^{(1,3,4)} A^{(1,2,4)}\right) \\
& =\min \{r(A)-r(A B), p-r(B)\} .
\end{aligned}
$$

Hence, the following two statements are equivalent:

(i) $r\left(B^{(1,3,4)} A^{(1,2,4)}\right)=r(A B)$ holds for all $B^{(1,3,4)}$ and $A^{(1,2,4)}$.

(ii) $r(A B)=r(A)$ or $r(B)=p$.

(11) The spread of the rank of $B^{(1,3,4)} A^{(1,2,3)}$ is

$$
\begin{aligned}
& \max _{B^{(1,3,4)}, A^{(1,2,3)}} r\left(B^{(1,3,4)} A^{(1,2,3)}\right)-\min _{B^{(1,3,4)}, B^{(1,2,3)}} r\left(B^{(1,3,4)} A^{(1,2,3)}\right) \\
& =\min \{p-r(A)-r(B)+r(M), r(M)-r(B)\} .
\end{aligned}
$$

Hence, the following two statements are equivalent:

(i) $r\left(B^{(1,3,4)} A^{(1,2,3)}\right)=r(A B)$ holds for all $B^{(1,3,4)}$ and $A^{(1,2,3)}$.

(ii) $r(M)=r(A)+r(B)-p$ or $\mathscr{R}\left(A^{*}\right) \subseteq \mathscr{R}(B)$. 
(12) The spread of the rank of $B^{(1,3,4)} A^{(1,4)}$ is

$$
\begin{aligned}
& \max _{B^{(1,3,4)}, A^{(1,4)}} r\left(B^{(1,3,4)} A^{(1,4)}\right)-\min _{B^{(1,3,4)}, A^{(1,4)}} r\left(B^{(1,3,4)} A^{(1,4)}\right) \\
& =\min \{m-r(A B), n-r(A B), p-r(A B), m+p-r(A)-r(B)\} .
\end{aligned}
$$

Hence, the following two statements are equivalent:

(i) $r\left(B^{(1,3,4)} A^{(1,4)}\right)=r(A B)$ holds for all $B^{(1,3,4)}$ and $A^{(1,4)}$.

(ii) $r(A B)=m$ or $r(A B)=n$ or $r(A B)=p$ or $r(A)+r(B)=m+p$.

(13) The spread of the rank of $B^{(1,3,4)} A^{(1,3)}$ is

$$
\begin{aligned}
& \max _{B^{(1,3,4)}, A^{(1,3)}} r\left(B^{(1,3,4)} A^{(1,3)}\right)-\min _{B^{(1,3,4)}, A^{(1,3)}} r\left(B^{(1,3,4)} A^{(1,3)}\right) \\
&=\min \{m-r(A)-r(B)+r(M), n-r(A)-r(B)+r(M), \\
&p-r(A)-r(B)+r(M)\} .
\end{aligned}
$$

Hence, the following two statements are equivalent:

(i) $r\left(B^{(1,3,4)} A^{(1,3)}\right)=r(A B)$ holds for all $B^{(1,3,4)}$ and $A^{(1,3)}$.

(ii) $r(A)+r(B)-r(M)=\min \{m, n, p\}$.

(14) The spread of the rank of $B^{(1,3,4)} A^{(1,2)}$ is

$$
\begin{gathered}
\max _{B^{(1,3,4)}, A^{(1,2)}} r\left(B^{(1,3,4)} A^{(1,2)}\right)-\min _{B^{(1,3,4)}, A^{(1,2)}} r\left(B^{(1,3,4)} A^{(1,2)}\right) \\
=\min \{p-r(A)-r(B)+r(M), r(M)-r(B)\} .
\end{gathered}
$$

Hence, the following two statements are equivalent:

(i) $r\left(B^{(1,3,4)} A^{(1,2)}\right)=r(A B)$ holds for all $B^{(1,3,4)}$ and $A^{(1,2)}$.

(ii) $r(M)=r(A)+r(B)-p$ or $\mathscr{R}\left(A^{*}\right) \subseteq \mathscr{R}(B)$.

(15) The spread of the rank of $B^{(1,3,4)} A^{(1)}$ is

$$
\begin{aligned}
\max _{B^{(1,3,4)}, A^{(1)}} r\left(B^{(1,3,4)} A^{(1)}\right)-\min _{B^{(1,3,4)}, A^{(1)}} r\left(B^{(1,3,4)} A^{(1)}\right) \\
=\min \{m-r(A)-r(B)+r(M), n-r(A)-r(B)+r(M), \\
\quad p-r(A)-r(B)+r(M)\} .
\end{aligned}
$$

Hence, the following two statements are equivalent:

(i) $r\left(B^{(1,3,4)} A^{(1)}\right)=r(A B)$ holds for all $B^{(1,3,4)}$ and $A^{(1)}$.

(ii) $r(A)+r(B)-r(M)=\min \{m, n, p\}$.

(16) The spread of the rank of $B^{(1,2,4)} A^{\dagger}$ is

$$
\max _{B^{(1,2,4)}} r\left(B^{(1,2,4)} A^{\dagger}\right)-\min _{B^{(1,2,4)}} r\left(B^{(1,2,4)} A^{\dagger}\right)=\min \{r(M)-r(A), r(M)-r(B)\} .
$$

Hence, the following two statements are equivalent:

(i) $r\left(B^{(1,2,4)} A^{\dagger}\right)=r(A B)$ holds for all $B^{(1,2,4)}$ and $A^{\dagger}$.

(ii) $\mathscr{R}\left(A^{*}\right) \subseteq \mathscr{R}(B)$ or $\mathscr{R}\left(A^{*}\right) \supseteq \mathscr{R}(B)$. 
(17) The spread of the rank of $B^{(1,2,4)} A^{(1,3,4)}$ is

$$
\begin{aligned}
& \max _{B^{(1,2,4)}, A^{(1,3,4)}} r\left(B^{(1,2,4)} A^{(1,3,4)}\right)-\min _{B^{(1,2,4)}, A^{(1,3,4)}} r\left(B^{(1,2,4)} A^{(1,3,4)}\right) \\
& =\min \{m-r(A)-r(B)+r(M), r(M)-r(A)\} .
\end{aligned}
$$

Hence, the following two statements are equivalent:

(i) $r\left(B^{(1,2,4)} A^{(1,3,4)}\right)=r(A B)$ holds for all $B^{(1,2,4)}$ and $A^{(1,3,4)}$.

(ii) $r(M)=r(A)+r(B)-m$ or $\mathscr{R}\left(A^{*}\right) \supseteq \mathscr{R}(B)$.

(18) The spread of the rank of $B^{(1,2,4)} A^{(1,2,4)}$ is

$$
\begin{aligned}
& \max _{B^{(1,2,4)}, A^{(1,2,4)}} r\left(B^{(1,2,4)} A^{(1,2,4)}\right)-\min _{B^{(1,2,4)}, A^{(1,2,4)}} r\left(B^{(1,2,4)} A^{(1,2,4)}\right) \\
& =\min \{r(M)-r(A), r(M)-r(B)\} .
\end{aligned}
$$

Hence, the following two statements are equivalent:

(i) $r\left(B^{(1,2,4)} A^{(1,2,4)}\right)=r(A B)$ holds for all $B^{(1,2,4)}$ and $A^{(1,2,4)}$.

(ii) $\mathscr{R}\left(A^{*}\right) \subseteq \mathscr{R}(B)$ or $\mathscr{R}\left(A^{*}\right) \supseteq \mathscr{R}(B)$.

(19) The spread of the rank of $B^{(1,2,4)} A^{(1,2,3)}$ is

$$
\begin{aligned}
& \max _{B^{(1,2,4)}, A^{(1,2,3)}} r\left(B^{(1,2,4)} A^{(1,2,3)}\right)-\min _{B^{(1,2,4)}, A^{(1,2,3)}} r\left(B^{(1,2,4)} A^{(1,2,3)}\right) \\
& =\min \{r(A), r(B), n-r(A), n-r(B)\} .
\end{aligned}
$$

Hence, the following two statements are equivalent:

(i) $r\left(B^{(1,2,4)} A^{(1,2,3)}\right)=r(A B)$ for all $B^{(1,2,4)}$ and $A^{(1,2,3)}$.

(ii) $A=0$ or $B=0$ or $r(A)=n$ or $r(B)=n$.

(20) The spread of the rank of $B^{(1,2,4)} A^{(1,4)}$ is

$$
\begin{gathered}
\max _{B^{(1,2,4)}, A^{(1,4)}} r\left(B^{(1,2,4)} A^{(1,4)}\right)-\min _{B^{(1,2,4)}, A^{(1,4)}} r\left(B^{(1,2,4)} A^{(1,4)}\right) \\
=\min \{m-r(A)-r(B)+r(M), r(M)-r(A)\} .
\end{gathered}
$$

Hence, the following two statements are equivalent:

(i) $r\left(B^{(1,2,4)} A^{(1,4)}\right)=r(A B)$ holds for all $B^{(1,2,4)}$ and $A^{(1,4)}$.

(ii) $r(M)=r(A)+r(B)-m$ or $\mathscr{R}\left(A^{*}\right) \supseteq \mathscr{R}(B)$.

(21) The spread of the rank of $B^{(1,2,4)} A^{(1,3)}$ is

$$
\begin{gathered}
\max _{B^{(1,2,4)}, A^{(1,3)}} r\left(B^{(1,2,4)} A^{(1,3)}\right)-\min _{B^{(1,2,4)}, A^{(1,3)}} r\left(B^{(1,2,4)} A^{(1,3)}\right) \\
=\min \{m, n-r(A), r(B), m+n-r(A)-r(B)\} .
\end{gathered}
$$

Hence, the following two statements are equivalent:

(i) $r\left(B^{(1,2,4)} A^{(1,3)}\right)=r(A B)$ for all $B^{(1,2,4)}$ and $A^{(1,3)}$.

(ii) $r(A)=n$, or $B=0$, or $r(A)=m$ and $r(B)=n$. 
(22) The spread of the rank of $B^{(1,2,4)} A^{(1,2)}$ is

$$
\begin{aligned}
& \max _{B^{(1,2,4)}, A^{(1,2)}} r\left(B^{(1,2,4)} A^{(1,2)}\right)-\min _{B^{(1,2,4)}, A^{(1,2)}} r\left(B^{(1,2,4)} A^{(1,2)}\right) \\
& =\min \{r(A), r(B), n-r(A), n-r(B)\} .
\end{aligned}
$$

Hence, the following two statements are equivalent:

(i) $r\left(B^{(1,2,4)} A^{(1,2)}\right)=r(A B)$ holds for all $B^{(1,2,4)}$ and $A^{(1,2)}$.

(ii) $A=0$ or $B=0$ or $r(A)=n$ or $r(B)=n$.

(23) The spread of the rank of $B^{(1,2,4)} A^{(1)}$ is

$$
\begin{gathered}
\max _{B^{(1,2,4)}, A^{(1)}} r\left(B^{(1,2,4)} A^{(1)}\right)-\min _{B^{(1,2,4)}, A^{(1)}} r\left(B^{(1,2,4)} A^{(1)}\right) \\
=\min \{m, n-r(A), r(B), m+n-r(A)-r(B)\} .
\end{gathered}
$$

Hence, the following two statements are equivalent:

(i) $r\left(B^{(1,2,4)} A^{(1)}\right)=r(A B)$ holds for all $B^{(1,2,4)}$ and $A^{(1)}$.

(ii) $r(A)=n$, or $B=0$, or $r(A)=m$ and $r(B)=n$.

(24) $r\left(B^{(1,2,3)} A^{\dagger}\right)=r(A B)$ holds for all $B^{(1,2,3)}$ and $A^{\dagger}$.

(25) The spread of the rank of $B^{(1,2,3)} A^{(1,3,4)}$ is

$$
\begin{aligned}
& \max _{B^{(1,2,3)}, A^{(1,3,4)}} r\left(B^{(1,2,3)} A^{(1,3,4)}\right)-\min _{B^{(1,2,3)}, A^{(1,3,4)}} r\left(B^{(1,2,3)} A^{(1,3,4)}\right) \\
& =\min \{r(B)-r(A B), m-r(A)\} .
\end{aligned}
$$

Hence, the following two statements are equivalent:

(i) $r\left(B^{(1,2,3)} A^{(1,3,4)}\right)=r(A B)$ holds for all $B^{(1,2,3)}$ and $A^{(1,3,4)}$.

(ii) $r(A B)=r(B)$ or $r(A)=m$.

(26) $r\left(B^{(1,2,3)} A^{(1,2,4)}\right)=r(A B)$ holds for all $B^{(1,2,3)}$ and $A^{(1,2,4)}$.

(27) The spread of the rank of $B^{(1,2,3)} A^{(1,2,3)}$ is

$$
\begin{aligned}
& \max _{B^{(1,2,3)}, A^{(1,2,3)}} r\left(B^{(1,2,3)} A^{(1,2,3)}\right)-\min _{B^{(1,2,3)}, A^{(1,2,3)}} r\left(B^{(1,2,3)} A^{(1,2,3)}\right) \\
& =\min \{r(M)-r(A), r(M)-r(B)\} .
\end{aligned}
$$

Hence, the following two statements are equivalent:

(i) $r\left(B^{(1,2,3)} A^{(1,2,3)}\right)=r(A B)$ holds for all $B^{(1,2,3)}$ and $A^{(1,2,3)}$.

(ii) $\mathscr{R}\left(A^{*}\right) \subseteq \mathscr{R}(B)$ or $\mathscr{R}\left(A^{*}\right) \supseteq \mathscr{R}(B)$.

(28) The spread of the rank of $B^{(1,2,3)} A^{(1,4)}$ is

$$
\begin{aligned}
& \max _{B^{(1,2,3)}, A^{(1,4)}} r\left(B^{(1,2,3)} A^{(1,4)}\right)-\min _{B^{(1,2,3)}, A^{(1,4)}} r\left(B^{(1,2,3)} A^{(1,4)}\right) \\
& =\min \{r(B)-r(A B), m-r(A)\} .
\end{aligned}
$$

Hence, the following two statements are equivalent:

(i) $r\left(B^{(1,2,3)} A^{(1,4)}\right)=r(A B)$ for all $B^{(1,2,3)}$ and $A^{(1,4)}$.

(ii) $r(A B)=r(B)$ or $r(A)=m$. 
(29) The spread of the rank of $B^{(1,2,3)} A^{(1,3)}$ is

$$
\begin{gathered}
\max _{B^{(1,2,3)}, A^{(1,3)}} r\left(B^{(1,2,3)} A^{(1,3)}\right)-\min _{B^{(1,2,3)}, A^{(1,3)}} r\left(B^{(1,2,3)} A^{(1,3)}\right) \\
=\min \{m-r(A)-r(B)+r(M), r(M)-r(A)\} .
\end{gathered}
$$

Hence, the following two statements are equivalent:

(i) $r\left(B^{(1,2,3)} A^{(1,3)}\right)=r(A B)$ holds for all $B^{(1,2,3)}$ and $A^{(1,3)}$.

(ii) $r(M)=r(A)+r(B)-m$ or $\mathscr{R}\left(A^{*}\right) \supseteq \mathscr{R}(B)$.

(30) The spread of the rank of $B^{(1,2,3)} A^{(1,2)}$ is

$$
\begin{aligned}
& \max _{B^{(1,2,3)}, A^{(1,2)}} r\left(B^{(1,2,3)} A^{(1,2)}\right)-\min _{B^{(1,2,3)}, A^{(1,2)}} r\left(B^{(1,2,3)} A^{(1,2)}\right) \\
& =\min \{r(M)-r(A), r(M)-r(B)\} .
\end{aligned}
$$

Hence, the following two statements are equivalent:

(i) $r\left(B^{(1,2,3)} A^{(1,2)}\right)=r(A B)$ holds for all $B^{(1,2,3)}$ and $A^{(1,2)}$.

(ii) $\mathscr{R}\left(A^{*}\right) \subseteq \mathscr{R}(B)$ or $\mathscr{R}\left(A^{*}\right) \supseteq \mathscr{R}(B)$.

(31) The spread of the rank of $B^{(1,2,3)} A^{(1)}$ is

$$
\begin{gathered}
\max _{B^{(1,2,3)}, A^{(1)}} r\left(B^{(1,2,3)} A^{(1)}\right)-\min _{B^{(1,2,3)}, A^{(1)}} r\left(B^{(1,2,3)} A^{(1)}\right) \\
=\min \{m-r(A)-r(B)+r(M), r(M)-r(A)\} .
\end{gathered}
$$

Hence, the following two statements are equivalent:

(i) $r\left(B^{(1,2,3)} A^{(1)}\right)=r(A B)$ for all $B^{(1,2,3)}$ and $A^{(1)}$.

(ii) $r(M)=r(A)+r(B)-m$ or $\mathscr{R}\left(A^{*}\right) \supseteq \mathscr{R}(B)$.

(32) The spread of the rank of $B^{(1,4)} A^{\dagger}$ is

$$
\begin{aligned}
& \max _{B^{(1,4)}} r\left(B^{(1,4)} A^{\dagger}\right)-\min _{B^{(1,4)}} r\left(B^{(1,4)} A^{\dagger}\right) \\
& \quad=\min \{p-r(A)-r(B)+r(M), r(M)-r(B)\} .
\end{aligned}
$$

Hence, the following two statements are equivalent:

(i) $r\left(B^{(1,4)} A^{\dagger}\right)=r(A B)$ holds for all $B^{(1,4)}$

(ii) $r(M)=r(A)+r(B)-p$ or $\mathscr{R}\left(A^{*}\right) \subseteq \mathscr{R}(B)$.

(33) The spread of the rank of $B^{(1,4)} A^{(1,3,4)}$ is

$$
\begin{aligned}
\max _{B^{(1,4)}, A^{(1,3,4)}} r\left(B^{(1,4)} A^{(1,3,4)}\right)-\min _{B^{(1,4)}, A^{(1,3,4)}} r\left(B^{(1,4)} A^{(1,3,4)}\right) \\
=\min \{m-r(A)-r(B)+r(M), n-r(A)-r(B)+r(M), \\
\quad p-r(A)-r(B)+r(M)\} .
\end{aligned}
$$

Hence, the following two statements are equivalent:

(i) $r\left(B^{(1,4)} A^{(1,3,4)}\right)=r(A B)$ holds for all $B^{(1,4)}$ and $A^{(1,3,4)}$.

(ii) $r(A)+r(B)-r(M)=\min \{m, n, p\}$. 
(34) The spread of the rank of $B^{(1,4)} A^{(1,2,4)}$ is

$$
\begin{gathered}
\max _{B^{(1,4)}, A^{(1,2,4)}} r\left(B^{(1,4)} A^{(1,2,4)}\right)-\min _{B^{(1,4)}, A^{(1,2,4)}} r\left(B^{(1,4)} A^{(1,2,4)}\right) \\
=\min \{p-r(A)-r(B)+r(M), r(M)-r(B)\} .
\end{gathered}
$$

Hence, the following two statements are equivalent:

(i) $r\left(B^{(1,4)} A^{(1,2,4)}\right)=r(A B)$ holds for all $B^{(1,4)}$ and $A^{(1,2,4)}$.

(ii) $r(M)=r(A)+r(B)-p$ or $\mathscr{R}\left(A^{*}\right) \subseteq \mathscr{R}(B)$.

(35) The spread of the rank of $B^{(1,4)} A^{(1,2,3)}$ is

$$
\begin{gathered}
\max _{B^{(1,4)}, A^{(1,2,3)}} r\left(B^{(1,4)} A^{(1,2,3)}\right)-\min _{B^{(1,4)}, A^{(1,2,3)}} r\left(B^{(1,4)} A^{(1,2,3)}\right) \\
=\min \{p, r(A), n-r(B), n+p-r(A)-r(B)\} .
\end{gathered}
$$

Hence, the following two statements are equivalent:

(i) $r\left(B^{(1,4)} A^{(1,2,3)}\right)=r(A B)$ holds for all $B^{(1,4)}$ and $A^{(1,2,3)}$.

(ii) $A=0$ or $r(B)=n$ or $r(A)+r(B)=n+p$

(36) The spread of the rank of $B^{(1,4)} A^{(1,4)}$ is

$$
\begin{aligned}
\max _{B^{(1,4)}, A^{(1,4)}} & r\left(B^{(1,4)} A^{(1,4)}\right)-\min _{B^{(1,4)}, A^{(1,4)}} r\left(B^{(1,4)} A^{(1,4)}\right) \\
= & \min \{m-r(A)-r(B)+r(M), n-r(A)-r(B)+r(M), \\
& p-r(A)-r(B)+r(M)\} .
\end{aligned}
$$

Hence, the following two statements are equivalent:

(i) $r\left(B^{(1,4)} A^{(1,4)}\right)=r(A B)$ holds for all $B^{(1,4)}$ and $A^{(1,4)}$.

(ii) $r(A)+r(B)-r(M)=\min \{m, n, p\}$.

(37) The spread of the rank of $B^{(1,4)} A^{(1,3)}$ is

$$
\begin{aligned}
& \max _{B^{(1,4)}, A^{(1,3)}} r\left(B^{(1,4)} A^{(1,3)}\right)-\min _{B^{(1,4)}, A^{(1,3)}} r\left(B^{(1,4)} A^{(1,3)}\right) \\
& =\min \{m, n, p, m+n-r(A)-r(B), 2 n-r(A)-r(B), n+p-r(A)-r(B)\} .
\end{aligned}
$$

Hence, the following two statements are equivalent:

(i) $r\left(B^{(1,4)} A^{(1,3)}\right)=r(A B)$ holds for all $B^{(1,4)}$ and $A^{(1,3)}$.

(ii) $r(A)+r(B)=\min \{m+n, 2 n, n+p\}$.

(38) The spread of the rank of $B^{(1,4)} A^{(1,2)}$ is

$$
\begin{aligned}
& \max _{B^{(1,4)}, A^{(1,2)}} r\left(B^{(1,4)} A^{(1,2)}\right)-\min _{B^{(1,4)}, A^{(1,2)}} r\left(B^{(1,4)} A^{(1,2)}\right) \\
& =\min \{p, r(A), n-r(B), n+p-r(A)-r(B)\} .
\end{aligned}
$$

Hence, the following two statements are equivalent:

(i) $r\left(B^{(1,4)} A^{(1,2)}\right)=r(A B)$ holds for all $B^{(1,4)}$ and $A^{(1,2)}$.

(ii) $A=0$, or $r(B)=n$, or $r(A)+r(B)=n+p$. 
(39) The spread of the rank of $B^{(1,4)} A^{(1)}$ is

$$
\begin{aligned}
\max _{B^{(1,4)}, A^{(1)}} r\left(B^{(1,4)} A^{1)}\right)-\min _{B^{(1,4)}, A^{(1)}} r\left(B^{(1,4)} A^{(1)}\right) \\
=\min \{m, n, p, m+n-r(A)-r(B), 2 n-r(A)-r(B), \\
\quad n+p-r(A)-r(B)\} .
\end{aligned}
$$

Hence, the following two statements are equivalent:

(i) $r\left(B^{(1,4)} A^{(1)}\right)=r(A B)$ for all $B^{(1,4)}$ and $A^{(1)}$.

(ii) $r(A)+r(B)=\min \{m+n, 2 n, n+p\}$.

(40) The spread of the rank of $B^{(1,3)} A^{\dagger}$ is

$$
\max _{B^{(1,3)}} r\left(B^{(1,3)} A^{\dagger}\right)-\min _{B^{(1,3)}} r\left(B^{(1,3)} A^{\dagger}\right)=\min \{r(A)-r(A B), p-r(B)\} .
$$

Hence, the following two statements are equivalent:

(i) $r\left(B^{(1,3)} A^{\dagger}\right)=r(A B)$ holds for all $B^{(1,3)}$.

(ii) $r(A B)=r(A)$ or $r(B)=p$.

(41) The spread of the rank of $B^{(1,3)} A^{(1,3,4)}$ is

$$
\begin{aligned}
& \max _{B^{(1,3)}, A^{(1,3,4)}} r\left(B^{(1,3)} A^{(1,3,4)}\right)-\min _{B^{(1,3)}, A^{(1,3,4)}} r\left(B^{(1,3)} A^{(1,3,4)}\right) \\
& =\min \{m-r(A B), n-r(A B), p-r(A B), m+p-r(A)-r(B)\} .
\end{aligned}
$$

Hence, the following two statements are equivalent:

(i) $r\left(B^{(1,3)} A^{(1,3,4)}\right)=r(A B)$ holds for all $B^{(1,3)}$ and $A^{(1,3,4)}$.

(ii) $r(A B)=m$ or $r(A B)=n$ or $r(A B)=p$ or $r(A)+r(B)=m+p$.

(42) The spread of the rank of $B^{(1,3)} A^{(1,2,4)}$ is

$$
\begin{aligned}
& \max _{B^{(1,3)}, A^{(1,2,4)}} r\left(B^{(1,3)} A^{(1,2,4)}\right)-\min _{B^{(1,3)}, A^{(1,2,4)}} r\left(B^{(1,3)} A^{(1,2,4)}\right) \\
& =\min \{r(A)-r(A B), p-r(B)\} .
\end{aligned}
$$

Hence, the following two statements are equivalent:

(i) $r\left(B^{(1,3)} A^{(1,2,4)}\right)=r(A B)$ holds for all $B^{(1,3)}$ and $A^{(1,2,4)}$.

(ii) $r(A B)=r(A)$ or $r(B)=p$.

(43) The spread of the rank of $B^{(1,3)} A^{(1,2,3)}$ is

$$
\begin{gathered}
\max _{B^{(1,3)}, A^{(1,2,3)}} r\left(B^{(1,3)} A^{(1,2,3)}\right)-\min _{B^{(1,3)}, A^{(1,2,3)}} r\left(B^{(1,3)} A^{(1,2,3)}\right) \\
=\min \{p-r(A)-r(B)+r(M), r(M)-r(B)\} .
\end{gathered}
$$

Hence, the following two statements are equivalent:

(i) $r\left(B^{(1,3)} A^{(1,2,3)}\right)=r(A B)$ holds for all $B^{(1,3)}$ and $A^{(1,2,3)}$.

(ii) $r(M)=r(A)+r(B)-p$ or $\mathscr{R}\left(A^{*}\right) \subseteq \mathscr{R}(B)$. 
(44) The spread of the rank of $B^{(1,3)} A^{(1,4)}$ is

$$
\begin{aligned}
& \max _{B^{(1,3)}, A^{(1,4)}} r\left(B^{(1,3)} A^{(1,4)}\right)-\min _{B^{(1,3)}, A^{(1,4)}} r\left(B^{(1,3)} A^{(1,4)}\right) \\
& \quad=\min \{m-r(A B), n-r(A B), p-r(A B), m+p-r(A)-r(B)\} .
\end{aligned}
$$

Hence, the following two statements are equivalent:

(i) $r\left(B^{(1,3)} A^{(1,4)}\right)=r(A B)$ holds for all $B^{(1,3)}$ and $A^{(1,4)}$.

(ii) $r(A B)=m$ or $r(A B)=n$ or $r(A B)=p$ or $r(A)+r(B)=m+p$.

(45) The spread of the rank of $B^{(1,3)} A^{(1,3)}$ is

$$
\begin{aligned}
\max _{B^{(1,3)}, A^{(1,3)}} r\left(B^{(1,3)} A^{1,3)}\right)-\min _{B^{(1,3)}, A^{(1,3)}} r\left(B^{(1,3)} A^{(1,3)}\right) \\
=\min \{m-r(A)-r(B)+r(M), n-r(A)-r(B)+r(M), \\
\quad p-r(A)-r(B)+r(M)\} .
\end{aligned}
$$

Hence, the following two statements are equivalent:

(i) $r\left(B^{(1,3)} A^{(1,3)}\right)=r(A B)$ holds for all $B^{(1,3)}$ and $A^{(1,3)}$.

(ii) $r(A)+r(B)-r(M)=\min \{m, n, p\}$.

(46) The spread of the rank of $B^{(1,3)} A^{(1,2)}$ is

$$
\begin{gathered}
\max _{B^{(1,3)}, A^{(1,2)}} r\left(B^{(1,3)} A^{(1,2)}\right)-\min _{B^{(1,3)}, A^{(1,2)}} r\left(B^{(1,3)} A^{(1,2)}\right) \\
=\min \{p-r(A)-r(B)+r(M), r(M)-r(B)\} .
\end{gathered}
$$

Hence, the following two statements are equivalent:

(i) $r\left(B^{(1,3)} A^{(1,2)}\right)=r(A B)$ holds for all $B^{(1,3)}$ and $A^{(1,2)}$.

(ii) $r(M)=r(A)+r(B)-p$ or $\mathscr{R}\left(A^{*}\right) \subseteq \mathscr{R}(B)$.

(47) The spread of the rank of $B^{(1,3)} A^{(1)}$ is

$$
\begin{aligned}
\max _{B^{(1,3)}, A^{(1)}} r\left(B^{(1,3)} A^{(1)}\right)-\min _{B^{(1,3)}, A^{(1)}} r\left(B^{(1,3)} A^{(1)}\right) \\
=\min \{m-r(A)-r(B)+r(M), n-r(A)-r(B)+r(M), \\
\quad p-r(A)-r(B)+r(M)\} .
\end{aligned}
$$

Hence, the following two statements are equivalent:

(i) $r\left(B^{(1,3)} A^{(1)}\right)=r(A B)$ holds for all $B^{(1,3)}$ and $A^{(1)}$.

(ii) $r(A)+r(B)-r(M)=\min \{m, n, p\}$.

(48) The spread of the rank of $B^{(1,2)} A^{\dagger}$ is

$$
\max _{B^{(1,2)}} r\left(B^{(1,2)} A^{\dagger}\right)-\min _{B^{(1,2)}} r\left(B^{(1,2)} A^{\dagger}\right)=\min \{r(M)-r(A), r(M)-r(B)\} .
$$

Hence, the following two statements are equivalent:

(i) $r\left(B^{(1,2)} A^{\dagger}\right)=r(A B)$ holds for all $B^{(1,2)}$ and $A^{\dagger}$.

(ii) $\mathscr{R}\left(A^{*}\right) \subseteq \mathscr{R}(B)$ or $\mathscr{R}\left(A^{*}\right) \supseteq \mathscr{R}(B)$. 
(49) The spread of the rank of $B^{(1,2)} A^{(1,3,4)}$ is

$$
\begin{gathered}
\max _{B^{(1,2)}, A^{(1,3,4)}} r\left(B^{(1,2)} A^{(1,3,4)}\right)-\min _{B^{(1,2)}, A^{(1,3,4)}} r\left(B^{(1,2)} A^{(1,3,4)}\right) \\
=\min \{m-r(A)-r(B)+r(M), r(M)-r(A)\} .
\end{gathered}
$$

Hence, the following two statements are equivalent:

(i) $r\left(B^{(1,2)} A^{(1,3,4)}\right)=r(A B)$ holds for all $B^{(1,2)}$ and $A^{(1,3,4)}$.

(ii) $r(M)=r(A)+r(B)-m$ or $\mathscr{R}\left(A^{*}\right) \supseteq \mathscr{R}(B)$.

(50) The spread of the rank of $B^{(1,2)} A^{(1,2,4)}$ is

$$
\begin{aligned}
& \max _{B^{(1,2)}, A^{(1,2,4)}} r\left(B^{(1,2)} A^{(1,2,4)}\right)-\min _{B^{(1,2)}, A^{(1,2,4)}} r\left(B^{(1,2)} A^{(1,2,4)}\right) \\
& =\min \{r(M)-r(A), r(M)-r(B)\} .
\end{aligned}
$$

Hence, the following two statements are equivalent:

(i) $r\left(B^{(1,2)} A^{(1,2,4)}\right)=r(A B)$ holds for all $B^{(1,2)}$ and $A^{(1,2,4)}$.

(ii) $\mathscr{R}\left(A^{*}\right) \subseteq \mathscr{R}(B)$ or $\mathscr{R}\left(A^{*}\right) \supseteq \mathscr{R}(B)$.

(51) The spread of the rank of $B^{(1,2)} A^{(1,2,3)}$ is

$$
\begin{aligned}
& \max _{B^{(1,2)}, A^{(1,2,3)}} r\left(B^{(1,2)} A^{(1,2,3)}\right)-\min _{B^{(1,2)}, A^{(1,2,3)}} r\left(B^{(1,2)} A^{(1,2,3)}\right) \\
& \quad=\min \{r(A), r(B), n-r(A), n-r(B)\} .
\end{aligned}
$$

Hence, the following two statements are equivalent:

(i) $r\left(B^{(1,2)} A^{(1,2,3)}\right)=r(A B)$ holds for all $B^{(1,2)}$ and $A^{(1,2,3)}$.

(ii) $A=0$ or $B=0$ or $r(A)=n$ or $r(B)=n$.

(52) The spread of the rank of $B^{(1,2)} A^{(1,4)}$ is

$$
\begin{gathered}
\max _{B^{(1,2)}, A^{(1,4)}} r\left(B^{(1,2)} A^{(1,4)}\right)-\min _{B^{(1,2)}, A^{(1,4)}} r\left(B^{(1,2)} A^{(1,4)}\right) \\
=\min \{m-r(A)-r(B)+r(M), r(M)-r(A)\} .
\end{gathered}
$$

Hence, the following two statements are equivalent:

(i) $r\left(B^{(1,2)} A^{(1,4)}\right)=r(A B)$ holds for all $B^{(1,2)}$ and $A^{(1,4)}$.

(ii) $r(M)=r(A)+r(B)-m$ or $\mathscr{R}\left(A^{*}\right) \supseteq \mathscr{R}(B)$.

(53) The spread of the rank of $B^{(1,2)} A^{(1,3)}$ is

$$
\begin{gathered}
\max _{B^{(1,2)}, A^{(1,3)}} r\left(B^{(1,2)} A^{(1,3)}\right)-\min _{B^{(1,2)}, A^{(1,3)}} r\left(B^{(1,2)} A^{(1,3)}\right) \\
=\min \{m, r(B), m+n-r(A)-r(B), n-r(A)\} .
\end{gathered}
$$

Hence, the following two statements are equivalent:

(i) $r\left(B^{(1,2)} A^{(1,3)}\right)=r(A B)$ holds for all $B^{(1,2)}$ and $A^{(1,3)}$.

(ii) $r(A)=n$ or $B=0$ or $r(A)+r(B)=m+n$. 
(54) The spread of the rank of $B^{(1,2)} A^{(1,2)}$ is

$$
\begin{aligned}
& \max _{B^{(1,2)}, A^{(1,2)}} r\left(B^{(1,2)} A^{(1,2)}\right)-\min _{B^{(1,2)}, A^{(1,2)}} r\left(B^{(1,2)} A^{(1,2)}\right) \\
& =\min \{r(A), r(B), n-r(A), n-r(B)\} .
\end{aligned}
$$

Hence, the following two statements are equivalent:

(i) $r\left(B^{(1,2)} A^{(1,2)}\right)=r(A B)$ holds for all $B^{(1,2)}$ and $A^{(1,2)}$.

(ii) $A=0$ or $B=0$ or $r(A)=n$ or $r(B)=n$.

(55) The spread of the rank of $B^{(1,2)} A^{(1)}$ is

$$
\begin{aligned}
& \max _{B^{(1,2)}, A^{(1)}} r\left(B^{(1,2)} A^{(1)}\right)-\min _{B^{(1,2)}, A^{(1)}} r\left(B^{(1,2)} A^{(1)}\right) \\
& \quad=\min \{m, n-r(A), r(B), m+n-r(A)-r(B)\} .
\end{aligned}
$$

Hence, the following two statements are equivalent:

(i) $r\left(B^{(1,2)} A^{(1)}\right)=r(A B)$ holds for all $B^{(1,2)}$ and $A^{(1)}$.

(ii) $r(A)=n$ or $B=0$ or $r(A)+r(B)=m+n$.

(56) The spread of the rank of $B^{(1)} A^{\dagger}$ is

$$
\max _{B^{(1)}} r\left(B^{(1)} A^{\dagger}\right)-\min _{B^{(1)}} r\left(B^{(1)} A^{\dagger}\right)=\min \{p-r(A)-r(B)+r(M), r(M)-r(B)\} .
$$

Hence, the following two statements are equivalent:

(i) $r\left(B^{(1)} A^{\dagger}\right)=r(A B)$ holds for all $B^{(1)}$ and $A^{\dagger}$.

(ii) $r(M)=r(A)+r(B)-p$ or $\mathscr{R}\left(A^{*}\right) \subseteq \mathscr{R}(B)$.

(57) The spread of the rank of $B^{(1)} A^{(1,3,4)}$ is

$$
\begin{aligned}
\max _{B^{(1)}, A^{(1,3,4)}} r\left(B^{(1)} A^{(1,3,4)}\right)-\min _{B^{(1)}, A^{(1,3,4)}} r\left(B^{(1)} A^{(1,3,4)}\right) \\
=\min \{m-r(A)-r(B)+r(M), n-r(A)-r(B)+r(M), \\
\quad p-r(A)-r(B)+r(M)\} .
\end{aligned}
$$

Hence, the following two statements are equivalent:

(i) $r\left(B^{(1)} A^{(1,3,4)}\right)=r(A B)$ for all $B^{(1)}$ and $A^{(1,3,4)}$.

(ii) $r(A)+r(B)-r(M)=\min \{m, n, p\}$.

(58) The spread of the rank of $B^{(1)} A^{(1,2,4)}$ is

$$
\begin{aligned}
& \max _{B^{(1)}, A^{(1,2,4)}} r\left(B^{(1)} A^{1,2,4)}\right)-\min _{B^{(1)}, A^{(1,2,4)}} r\left(B^{(1)} A^{(1,2,4)}\right) \\
& =\min \{p-r(A)-r(B)+r(M), r(M)-r(B)\} .
\end{aligned}
$$

Hence, the following two statements are equivalent:

(i) $r\left(B^{(1)} A^{(1,2,4)}\right)=r(A B)$ holds for all $B^{(1)}$ and $A^{(1,2,4)}$.

(ii) $r(M)=r(A)+r(B)-p$ or $\mathscr{R}\left(A^{*}\right) \subseteq \mathscr{R}(B)$. 
(59) The spread of the rank of $B^{(1)} A^{(1,2,3)}$ is

$$
\begin{gathered}
\max _{B^{(1)}, A^{(1,2,3)}} r\left(B^{(1)} A^{(1,2,3)}\right)-\min _{B^{(1)}, A^{(1,2,3)}} r\left(B^{(1)} A^{(1,2,3)}\right) \\
=\min \{p, r(A), n-r(B), n+p-r(A)-r(B)\} .
\end{gathered}
$$

Hence, the following two statements are equivalent:

(i) $r\left(B^{(1)} A^{(1,2,3)}\right)=r(A B)$ for all $B^{(1)}$ and $A^{(1,2,3)}$.

(ii) $A=0$ or $r(B)=n$ or $r(A)+r(B)=n+p$.

(60) The spread of the rank of $B^{(1)} A^{(1,4)}$ is

$$
\begin{aligned}
\max _{B^{(1)}, A^{(1,4)}} & r\left(B^{(1)} A^{(1,4)}\right)-\min _{B^{(1)}, A^{(1,4)}} r\left(B^{(1)} A^{(1,4)}\right) \\
= & \min \{m-r(A)-r(B)+r(M), b n-r(A)-r(B)+r(M), \\
& p-r(A)-r(B)+r(M)\} .
\end{aligned}
$$

Hence, the following two statements are equivalent:

(i) $r\left(B^{(1)} A^{(1,4)}\right)=r(A B)$ for all $B^{(1)}$ and $A^{(1,4)}$.

(ii) $r(A)+r(B)-r(M)=\min \{m, n, p\}$.

(61) The spread of the rank of $B^{(1)} A^{(1,3)}$ is

$$
\begin{aligned}
& \max _{B^{(1)}, A^{(1,3)}} r\left(B^{(1)} A^{(1,3)}\right)-\min _{B^{(1)}, A^{(1,3)}} r\left(B^{(1)} A^{(1,3)}\right) \\
& \quad=\min \{m, n, p, m+n-r(A)-r(B), 2 n-r(A)-r(B), n+p-r(A)-r(B)\} .
\end{aligned}
$$

Hence, the following two statements are equivalent:

(i) $r\left(B^{(1)} A^{(1,3)}\right)=r(A B)$ holds for all $B^{(1)}$ and $A^{(1,3)}$.

(ii) $r(A)+r(B)=\min \{m+n, 2 n, n+p\}$.

(62) The spread of the rank of $B^{(1)} A^{(1,2)}$ is

$$
\begin{aligned}
& \max _{B^{(1)}, A^{(1,2)}} r\left(B^{(1)} A^{(1,2)}\right)-\min _{B^{1)}, A^{(1,2)}} r\left(B^{(1)} A^{(1,2)}\right) \\
& =\min \{p, r(A), n-r(B), n+p-r(A)-r(B)\} .
\end{aligned}
$$

Hence, the following two statements are equivalent:

(i) $r\left(B^{(1)} A^{(1,2)}\right)=r(A B)$ for all $B^{(1)}$ and $A^{(1,2)}$.

(ii) $A=0$ or $r(B)=n$ or $r(A)+r(B)=n+p$.

(63) The spread of the rank of $B^{(1)} A^{(1)}$ is

$$
\begin{aligned}
& \max _{B^{(1)}, A^{(1)}} r\left(B^{(1)} A^{(1)}\right)-\min _{B^{(1)}, A^{(1)}} r\left(B^{(1)} A^{(1)}\right) \\
& \quad=\min \{m, n, p, m+n-r(A)-r(B), 2 n-r(A)-r(B), n+p-r(A)-r(B)\} .
\end{aligned}
$$

Hence, the following two statements are equivalent:

(i) $r\left(B^{(1)} A^{(1)}\right)=r(A B)$ holds for all $B^{(1)}$ and $A^{(1)}$.

(ii) $r(A)+r(B)=\min \{m+n, 2 n, n+p\}$. 
Corollary 2 Let $A \in \mathbb{C}^{m \times n}$ and $B \in \mathbb{C}^{n \times p}$ be given. Then, the following results hold.

(a) The following statements are equivalent:

(1) $r\left(B^{\dagger} A^{(1,2,3)}\right) \geq r(A B)$ holds for all $A^{(1,2,3)}$.

(2) $r\left(B^{\dagger} A^{(1,3)}\right) \geq r(A B)$ holds for all $A^{(1,3)}$.

(3) $r\left(B^{\dagger} A^{(1,2)}\right) \geq r(A B)$ holds for all $A^{(1,2)}$.

(4) $r\left(B^{\dagger} A^{(1)}\right) \geq r(A B)$ holds for all $A^{(1)}$.

(5) $r\left(B^{(1,3,4)} A^{(1,2,3)}\right) \geq r(A B)$ holds for all $B^{(1,3,4)}$ and $A^{(1,2,3)}$.

(6) $r\left(B^{(1,3,4)} A^{(1,3)}\right) \geq r(A B)$ holds for all $B^{(1,3,4)}$ and $A^{(1,3)}$.

(7) $r\left(B^{(1,3,4)} A^{(1,2)}\right) \geq r(A B)$ holds for all $B^{(1,3,4)}$ and $A^{(1,2)}$.

(8) $r\left(B^{(1,3,4)} A^{(1)}\right) \geq r(A B)$ holds for all $B^{(1,3,4)}$ and $A^{(1)}$.

(9) $r\left(B^{(1,2,4)} A^{\dagger}\right) \geq r(A B)$ holds for all $B^{(1,2,4)}$.

(10) $r\left(B^{(1,2,4)} A^{(1,3,4)}\right) \geq r(A B)$ holds for all $B^{(1,2,4)}$ and $A^{(1,3,4)}$.

(11) $r\left(B^{(1,2,4)} A^{(1,2,4)}\right) \geq r(A B)$ holds for all $B^{(1,2,4)}$ and $A^{(1,2,4)}$.

(12) $r\left(B^{(1,2,4)} A^{(1,4)}\right) \geq r(A B)$ holds for all $B^{(1,2,4)}$ and $A^{(1,4)}$.

(13) $r\left(B^{(1,2,3)} A^{(1,2,3)}\right) \geq r(A B)$ holds for all $B^{(1,2,3)}$ and $A^{(1,2,3)}$.

(14) $r\left(B^{(1,2,3)} A^{(1,3)}\right) \geq r(A B)$ holds for all $B^{(1,2,3)}$ and $A^{(1,3)}$.

(15) $r\left(B^{(1,2,3)} A^{(1,2)}\right) \geq r(A B)$ holds for all $B^{(1,2,3)}$ and $A^{(1,2)}$.

(16) $r\left(B^{(1,2,3)} A^{(1)}\right) \geq r(A B)$ holds for all $B^{(1,2,3)}$ and $A^{(1)}$.

(17) $r\left(B^{(1,4)} A^{\dagger}\right) \geq r(A B)$ holds for all $B^{(1,4)}$.

(18) $r\left(B^{(1,4)} A^{(1,3,4)}\right) \geq r(A B)$ holds for all $B^{(1,4)}$ and $A^{(1,3,4)}$.

(19) $r\left(B^{(1,4)} A^{(1,2,4)}\right) \geq r(A B)$ holds for all $B^{(1,4)}$ and $A^{(1,2,4)}$.

(20) $r\left(B^{(1,4)} A^{(1,4)}\right) \geq r(A B)$ holds for all $B^{(1,4)}$ and $A^{(1,4)}$.

(21) $r\left(B^{(1,3)} A^{(1,2,3)}\right) \geq r(A B)$ holds for all $B^{(1,3)}$ and $A^{(1,2,3)}$.

(22) $r\left(A^{(1,3)} A^{(1,3)}\right) \geq r(A B)$ holds for all $B^{(1,3)}$ and $A^{(1,3)}$.

(23) $r\left(B^{(1,3)} A^{(1,2)}\right) \geq r(A B)$ holds for all $B^{(1,3)}$ and $A^{(1,2)}$.

(24) $r\left(B^{(1,3)} A^{(1)}\right) \geq r(A B)$ holds for all $B^{(1,3)}$ and $A^{(1)}$.

(25) $r\left(B^{(1,2)} A^{\dagger}\right) \geq r(A B)$ holds for all $B^{(1,2)}$.

(26) $r\left(B^{(1,2)} A^{(1,3,4)}\right) \geq r(A B)$ holds for all $B^{(1,2)}$ and $A^{(1,3,4)}$.

(27) $r\left(B^{(1,2)} A^{(1,2,4)}\right) \geq r(A B)$ holds for all $B^{(1,2)}$ and $A^{(1,2,4)}$.

(28) $r\left(B^{(1,2)} A^{(1,4)}\right) \geq r(A B)$ holds for all $B^{(1,2)}$ and $A^{(1,4)}$.

(29) $r\left(B^{(1)} A^{\dagger}\right) \geq r(A B)$ holds for all $B^{(1)}$.

(30) $r\left(B^{(1)} A^{(1,3,4)}\right) \geq r(A B)$ holds for all $B^{(1)}$ and $A^{(1,3,4)}$.

(31) $r\left(B^{(1)} A^{(1,2,4)}\right) \geq r(A B)$ holds for all $B^{(1)}$ and $A^{(1,2,4)}$.

(32) $r\left(B^{(1)} A^{(1,4)} \geq r(A B)\right.$ holds for all $B^{(1)}$ and $A^{(1,4)}$.

(33) $r(M)=r(A)+r(B)-r(A B)$.

(b) The following statements are equivalent:

(1) $r\left(B^{(1,2,4)} A^{(1,2,3)}\right) \geq r(A B)$ holds for all $B^{(1,2,4)}$ and $A^{(1,2,3)}$.

(2) $r\left(B^{(1,2,4)} A^{(1,3)}\right) \geq r(A B)$ holds for all $B^{(1,2,4)}$ and $A^{(1,3)}$.

(3) $r\left(B^{(1,2,4)} A^{(1,2)}\right) \geq r(A B)$ holds for all $B^{(1,2,4)}$ and $A^{(1,2)}$.

(4) $r\left(B^{(1,2,4)} A^{(1)}\right) \geq r(A B)$ holds for all $B^{(1,2,4)}$ and $A^{(1)}$.

(5) $r\left(B^{(1,4)} A^{(1,2,3)}\right) \geq r(A B)$ holds for all $B^{(1,4)}$ and $A^{(1,2,3)}$.

(6) $r\left(B^{(1,4)} A^{(1,3)}\right) \geq r(A B)$ holds for all $B^{(1,4)}$ and $A^{(1,3)}$.

(7) $r\left(B^{(1,4)} A^{(1,2)}\right) \geq r(A B)$ holds for all $B^{(1,4)}$ and $A^{(1,2)}$.

(8) $r\left(B^{(1,4)} A^{(1)}\right) \geq r(A B)$ holds for all $B^{(1,4)}$ and $A^{(1)}$.

(9) $r\left(B^{(1,2)} A^{(1,2,3)}\right) \geq r(A B)$ holds for all $B^{(1,2)}$ and $A^{(1,2,3)}$.

(10) $r\left(B^{(1,2)} A^{(1,3)}\right) \geq r(A B)$ holds for all $B^{(1,2)}$ and $A^{(1,3)}$. 
(11) $r\left(B^{(1,2)} A^{(1,2)}\right) \geq r(A B)$ holds for all $B^{(1,2)}$ and $A^{(1,2)}$.

(12) $r\left(B^{(1,2)} A^{(1)}\right) \geq r(A B)$ holds for all $B^{(1,2)}$ and $A^{(1)}$.

(13) $r\left(B^{(1)} A^{(1,2,3)}\right) \geq r(A B)$ holds for all $B^{(1)}$ and $A^{(1,2,3)}$.

(14) $r\left(B^{(1)} A^{(1,3)}\right) \geq r(A B)$ holds for all $B^{(1)}$ and $A^{(1,3)}$.

(15) $r\left(B^{(1)} A^{(1,2)}\right) \geq r(A B)$ holds for all $B^{(1)}$ and $A^{(1,2)}$.

(16) $r\left(B^{(1)} A^{(1)}\right) \geq r(A B)$ holds for all $B^{(1)}$ and $A^{(1)}$.

(17) $A B=0$ or $r(A B)=r(A)+r(B)-n$.

Proof The proof follows from setting the minimum ranks of the corresponding $B^{(i, \ldots, j)} A^{(i, \ldots, j)}$ equal to $r(A B)$.

Corollary 3 Let $A \in \mathbb{C}^{m \times n}$ and $B \in \mathbb{C}^{n \times p}$ be given. Then the following results hold.

(a) The following statements are equivalent:

(1) There exists an $A^{(1,3,4)}$ such that $B^{\dagger} A^{(1,3,4)}=0$.

(2) $B^{\dagger} A^{(1,2,4)}=0$ holds for some/any $A^{(1,2,4)}$.

(3) There exists an $A^{(1,4)}$ such that $B^{\dagger} A^{(1,4)}=0$.

(4) There exists a $B^{(1,3,4)}$ such that $B^{(1,3,4)} A^{\dagger}=0$.

(5) There exist $B^{(1,3,4)}$ and $A^{(1,3,4)}$ such that $B^{(1,3,4)} A^{(1,3,4)}=0$.

(6) There exist $B^{(1,3,4)}$ and $A^{(1,2,4)}$ such that $B^{(1,3,4)} A^{(1,2,4)}=0$.

(7) There exist $B^{(1,3,4)}$ and $A^{(1,4)}$ such that $B^{(1,3,4)} A^{(1,4)}=0$.

(8) $B^{(1,2,3)} A^{\dagger}=0$ holds for some/any $B^{(1,2,3)}$.

(9) There exist $B^{(1,2,3)}$ and $A^{(1,3,4)}$ such that $B^{(1,2,3)} A^{(1,3,4)}=0$.

(10) $B^{(1,2,3)} A^{(1,2,4)}=0$ holds for somelany $B^{(1,2,3)}$ and $A^{(1,2,4)}$.

(11) There exist $B^{(1,2,3)}$ and $A^{(1,4)}$ such that $B^{(1,2,3)} A^{(1,4)}=0$.

(12) There exists a $B^{(1,3)}$ such that $B^{(1,3)} A^{\dagger}=0$.

(13) There exist $B^{(1,3)}$ and $A^{(1,3,4)}$ such that $B^{(1,3)} A^{(1,3,4)}=0$.

(14) There exist $B^{(1,3)}$ and $A^{(1,2,4)}$ such that $B^{(1,3)} A^{(1,2,4)}=0$.

(15) There exist $B^{(1,3)}$ and $A^{(1,4)}$ such that $B^{(1,3)} A^{(1,4)}=0$.

(16) $A B=0$.

(b) The following statements are equivalent:

(1) There exists an $A^{(1,2,3)}$ such that $B^{\dagger} A^{(1,2,3)}=0$.

(2) There exists an $A^{(1,3)}$ such that $B^{\dagger} A^{(1,3)}=0$.

(3) There exists an $A^{(1,2)}$ such that $B^{\dagger} A^{(1,2)}=0$.

(4) There exists an $A^{(1)}$ such that $B^{\dagger} A^{(1)}=0$.

(5) There exist $B^{(1,3,4)}$ and $A^{(1,2,3)}$ such that $B^{(1,3,4)} A^{(1,2,3)}=0$.

(6) There exist $B^{(1,3,4)}$ and $A^{(1,3)}$ such that $B^{(1,3,4)} A^{(1,3)}=0$.

(7) There exist $B^{(1,3,4)}$ and $A^{(1,2)}$ such that $B^{(1,3,4)} A^{(1,2)}=0$.

(8) There exist $B^{(1,3,4)}$ and $A^{(1)}$ such that $B^{(1,3,4)} A^{(1)}=0$.

(9) There exists a $B^{(1,2,4)}$ such that $B^{(1,2,4)} A^{\dagger}=0$.

(10) There exist $B^{(1,2,4)}$ and $A^{(1,3,4)}$ such that $B^{(1,2,4)} A^{(1,3,4)}=0$.

(11) There exist $B^{(1,2,4)}$ and $A^{(1,2,4)}$ such that $B^{(1,2,4)} A^{(1,2,4)}=0$.

(12) There exist $B^{(1,2,4)}$ and $A^{(1,4)}$ such that $B^{(1,2,4)} A^{(1,4)}=0$.

(13) There exist $B^{(1,2,3)}$ and $A^{(1,2,3)}$ such that $B^{(1,2,3)} A^{(1,2,3)}=0$.

(14) There exist $B^{(1,2,3)}$ and $A^{(1,3)}$ such that $B^{(1,2,3)} A^{(1,3)}=0$.

(15) There exist $B^{(1,2,3)}$ and $A^{(1,2)}$ such that $B^{(1,2,3)} A^{(1,2)}=0$.

(16) There exist $B^{(1,2,3)}$ and $A^{(1)}$ such that $B^{(1,2,3)} A^{(1)}=0$. 
(17) There exists a $B^{(1,4)}$ such that $B^{(1,4)} A^{\dagger}=0$.

(18) There exist $B^{(1,4)}$ and $A^{(1,3,4)}$ such that $B^{(1,4)} A^{(1,3,4)}=0$.

(19) There exist $B^{(1,4)}$ and $A^{(1,2,4)}$ such that $B^{(1,4)} A^{(1,2,4)}=0$.

(20) There exist $B^{(1,4)}$ and $A^{(1,4)}$ such that $B^{(1,4)} A^{(1,4)}=0$.

(21) There exist $B^{(1,3)}$ and $A^{(1,2,3)}$ such that $B^{(1,3)} A^{(1,2,3)}=0$.

(22) There exist $B^{(1,3)}$ and $A^{(1,3)}$ such that $B^{(1,3)} A^{(1,3)}=0$.

(23) There exist $B^{(1,3)}$ and $A^{(1,2)}$ such that $B^{(1,3)} A^{(1,2)}=0$.

(24) There exist $B^{(1,3)}$ and $A^{(1)}$ such that $B^{(1,3)} A^{(1)}=0$.

(25) There exists a $B^{(1,2)}$ such that $B^{(1,2)} A^{\dagger}=0$.

(26) There exist $B^{(1,2)}$ and $A^{(1,3,4)}$ such that $B^{(1,2)} A^{(1,3,4)}=0$.

(27) There exist $B^{(1,2)}$ and $A^{(1,2,4)}$ such that $B^{(1,2)} A^{(1,2,4)}=0$.

(28) There exist $B^{(1,2)}$ and $A^{(1,4)}$ such that $B^{(1,2)} A^{(1,4)}=0$.

(29) There exists a $B^{(1)}$ such that $B^{(1)} A^{\dagger}=0$.

(30) There exist $B^{(1)}$ and $A^{(1,3,4)}$ such that $B^{(1)} A^{(1,3,4)}=0$.

(31) There exist $B^{(1)}$ and $A^{(1,2,4)}$ such that $B^{(1)} A^{(1,2,4)}=0$.

(32) There exist $B^{(1)}$ and $A^{(1,4)}$ such that $B^{(1)} A^{(1,4)}=0$.

(33) $\mathscr{R}\left(A^{*}\right) \cap \mathscr{R}(B)=\{0\}$.

(c) The following statements are equivalent:

(1) There exist $B^{(1,2,4)}$ and $A^{(1,2,3)}$ such that $B^{(1,2,4)} A^{(1,2,3)}=0$.

(2) There exist $B^{(1,2,4)}$ and $A^{(1,3)}$ such that $B^{(1,2,4)} A^{(1,3)}=0$.

(3) There exist $B^{(1,2,4)}$ and $A^{(1,2)}$ such that $B^{(1,2,4)} A^{(1,2)}=0$.

(4) There exist $B^{(1,2,4)}$ and $A^{(1)}$ such that $B^{(1,2,4)} A^{(1)}=0$.

(5) There exist $B^{(1,4)}$ and $A^{(1,2,3)}$ such that $B^{(1,4)} A^{(1,2,3)}=0$.

(6) There exist $B^{(1,4)}$ and $A^{(1,3)}$ such that $B^{(1,4)} A^{(1,3)}=0$.

(7) There exist $B^{(1,4)}$ and $A^{(1,2)}$ such that $B^{(1,4)} A^{(1,2)}=0$.

(8) There exist $B^{(1,4)}$ and $A^{(1)}$ such that $B^{(1,4)} A^{(1)}=0$.

(9) There exist $B^{(1,2)}$ and $A^{(1,2,3)}$ such that $B^{(1,2)} A^{(1,2,3)}=0$.

(10) There exist $B^{(1,2)}$ and $A^{(1,3)}$ such that $B^{(1,2)} A^{(1,3)}=0$.

(11) There exist $B^{(1,2)}$ and $A^{(1,2)}$ such that $B^{(1,2)} A^{(1,2)}=0$.

(12) There exist $B^{(1,2)}$ and $A^{(1)}$ such that $B^{(1,2)} A^{(1)}=0$.

(13) There exist $B^{(1)}$ and $A^{(1,2,3)}$ such that $B^{(1)} A^{(1,2,3)}=0$.

(14) There exist $B^{(1)}$ and $A^{(1,3)}$ such that $B^{(1)} A^{(1,3)}=0$.

(15) There exist $B^{(1)}$ and $A^{(1,2)}$ such that $B^{(1)} A^{(1,2)}=0$.

(16) There exist $B^{(1)}$ and $A^{(1)}$ such that $B^{(1)} A^{(1)}=0$.

(17) $r(A)+r(B) \leq n$.

Proof The proof follows from setting the minimum ranks of the corresponding $B^{(i, \ldots, j)} A^{(i, \ldots, j)}$ equal to zero.

\section{Conclusions}

A huge amount of rank formulas associated with reverse-order laws of generalized inverses of products of matrices have been established since the 1990s, which played essential roles in revealing mechanisms of reverse-order laws. As one part of this ongoing work, we reconsidered in this paper products of generalized inverses of two matrices, established a group of exact forms for calculating the ranks of the products, and described many performances of the products via the rank formulas. All these results can serve as 
standard references in the investigation of various problems related to products of generalized inverses of matrices. Furthermore, there are many problems on establishing rank formulas for generalized inverses of matrix products and their applications. For instance,

(a) Establish analytical formulas for calculating (2.3)-(2.7), and use the formulas to derive necessary and sufficient conditions for (1.3)-(1.7) to hold.

(b) Establish analytical formulas for calculating the maximum and minimum ranks of the multiple matrix products in (3.65)-(3.108), and characterize the performances of these products via the rank formulas.

\section{Competing interests}

The author declares that he has no competing interests.

\section{Author's contributions}

The work as a whole is a contribution of the author.

\section{Acknowledgements}

The author thanks an anonymous referee for his/her helpful comments and suggestions on an earlier version of this paper. This work was supported by the National Natural Science Foundation of China (Grant No. 11271384).

Received: 23 April 2016 Accepted: 2 July 2016 Published online: 19 July 2016

\section{References}

1. Moore, EH: On the reciprocal of the general algebraic matrix. Bull. Am. Math. Soc. 26, 394-395 (1920)

2. Penrose, R: A generalized inverse for matrices. Proc. Camb. Philos. Soc. 51, 406-413 (1955)

3. Ben-Israel, A, Greville, TNE: Generalized Inverses: Theory and Applications, 2nd edn. Springer, New York (2003)

4. Campbell, SL, Meyer, CD Jr.: Generalized Inverses of Linear Transformations. Corrected reprint of the 1979 original. Dover, New York (1991)

5. Rao, CR, Mitra, SK: Generalized Inverse of Matrices and Its Applications. Wiley, New York (1971)

6. Arghiriade, E: Sur les matrices qui sont permutables avec leur inverse généralisée. Atti Accad. Naz. Lincei, Rend. Cl. Sci. Fis. Mat. Nat. (8) 35, 244-251 (1963)

7. Arghiriade, E: Remarques sur l'inverse généralisée d'un produit de matrices. Atti Accad. Naz. Lincei, Rend. Cl. Sci. Fis. Mat. Nat. (8) 42, 621-625 (1967)

8. Cvetković-llić, DS, Djikić, M: Various solutions to reverse order law problems. Linear Multilinear Algebra 64, 1207-1219 (2016)

9. Dinčić, NČ, Djordjević, DS: Basic reverse order law and its equivalencies. Aequ. Math. 85, 505-517 (2013)

10. Djordjević, DS: Unified approach to the reverse order rule for generalized inverses. Acta Sci. Math. 67, 761-776 (2001)

11. Galperin, AM, Waksman, Z: On pseudoinverses of operator products. Linear Algebra Appl. 33, 123-131 (1980)

12. Greville, TNE: Note on the generalized inverse of a matrix product. SIAM Rev. 8, 518-521 (1966)

13. Hartwig, RE: The reverse order law revisited. Linear Algebra Appl. 76, 241-246 (1986)

14. $\mathrm{Hu}, \mathrm{X}:$ A three-condition characterization of the Moore-Penrose generalized inverse. Am. Stat. 62, 216-218 (2008)

15. Izumino, S: The product of operators with closed range and an extension of the reverse order law. Tohoku Math. J. 34, 43-52 (1982)

16. Shinozaki, N, Sibuya, M: The reverse order law $(A B)^{-}=B^{-} A^{-}$. Linear Algebra Appl. 9, 29-40 (1974)

17. Shinozaki, N, Sibuya, M: Further results on the reverse-order law. Linear Algebra Appl. 27, 9-16 (1979)

18. Tian, Y: Using rank formulas to characterize equalities for Moore-Penrose inverses of matrix products. Appl. Math. Comput. 147, 581-600 (2004)

19. Tian, Y: On mixed-type reverse-order laws for the Moore-Penrose inverse of a matrix product. Int. J. Math. Math. Sci. 58, 3103-3116 (2004)

20. Tian, Y: The equivalence between $(A B)^{\dagger}=B^{\dagger} A^{\dagger}$ and other mixed-type reverse-order laws. Int. J. Math. Educ. Sci. Technol. 37, 331-339 (2006)

21. Werner, HJ: G-Inverse of matrix products. In: Schach, S, Trenkler, G (eds.) Data Analysis and Statistical Inference, pp. 531-546. Eul Verlag, Bergisch (1992)

22. Werner, HJ: When is $B^{-} A^{-}$a generalized inverse of $A B$ ? Linear Algebra Appl. 210, 255-263 (1994)

23. Yanai, $\mathrm{H}$ : Some generalized forms of least squares $g$-inverse, minimum norm $g$-inverse, and Moore-Pernrose inverse matrices. Comput. Stat. Data Anal. 10, 251-260 (1990)

24. Marsaglia, G, Styan, GPH: Equalities and inequalities for ranks of matrices. Linear Multilinear Algebra 2, 269-292 (1974)

25. Tian, Y: Reverse order laws for the generalized inverses of multiple matrix products. Linear Algebra Appl. 211, 85-100 (1994)

26. Tian, Y: More on maximal and minimal ranks of Schur complements with applications. Appl. Math. Comput. 152, 675-692 (2004)

27. Tian, Y: Extremal ranks of a quadratic matrix expression with applications. Linear Multilinear Algebra 59, 627-644 (2011) 